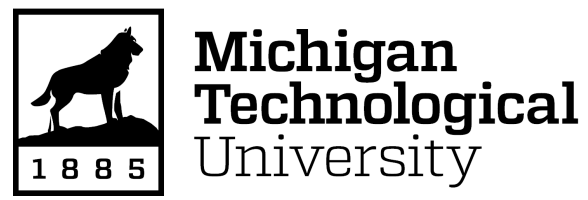

Michigan Technological University Digital Commons @ Michigan Tech

Dissertations, Master's Theses and Master's Reports

2017

\title{
MULTISCALE MODELING: THERMAL CONDUCTIVITY OF GRAPHENE/CYCLOALIPHATIC EPOXY COMPOSITES
}

Sorayot Chinkanjanarot

Michigan Technological University, schinkan@mtu.edu

Copyright 2017 Sorayot Chinkanjanarot

\section{Recommended Citation}

Chinkanjanarot, Sorayot, "MULTISCALE MODELING: THERMAL CONDUCTIVITY OF GRAPHENE/ CYCLOALIPHATIC EPOXY COMPOSITES", Open Access Dissertation, Michigan Technological University, 2017.

https://doi.org/10.37099/mtu.dc.etdr/491

Follow this and additional works at: https://digitalcommons.mtu.edu/etdr

Part of the Computational Engineering Commons, Computer-Aided Engineering and Design Commons, Other Mechanical Engineering Commons, and the Polymer and Organic Materials Commons 
MULTISCALE MODELING: THERMAL CONDUCTIVITY OF GRAPHENE/CYCLOALIPHATIC EPOXY COMPOSITES

\title{
By
}

Sorayot Chinkanjanarot

\begin{abstract}
A DISSERTATION
Submitted in partial fulfillment of the requirements for the degree of DOCTOR OF PHILOSOPHY

In Mechanical Engineering-Engineering Mechanics
\end{abstract}

MICHIGAN TECHNOLOGICAL UNIVERSITY

2017

(C) 2017 Sorayot Chinkanjanarot 
This dissertation has been approved in partial fulfillment of the requirements for the Degree of DOCTOR OF PHILOSOPHY in Mechanical Engineering-Engineering Mechanics.

Department of Mechanical Engineering-Engineering Mechanics

Dissertation Advisor: $\quad$ Gregory M. Odegard

Committee Member: Julia A. King

Committee Member: $\quad$ Ravindra Pandey

Committee Member: Gowtham $S$

Department Chair: $\quad$ William W. Predebon 


\section{TABLE OF CONTENTS}

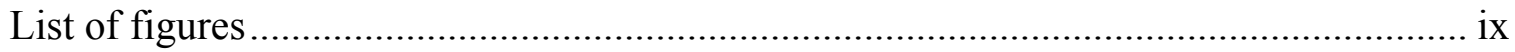

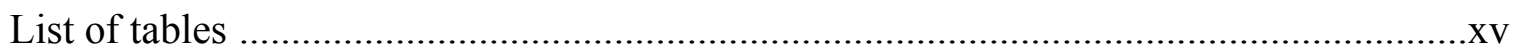

Preface $\quad$ (2)

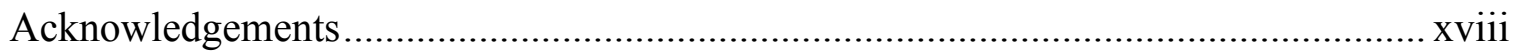

Abstract

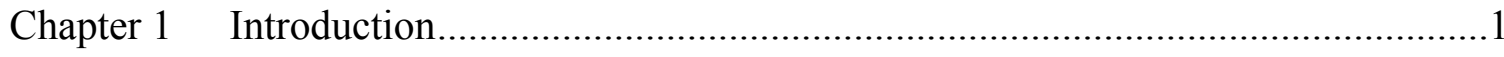

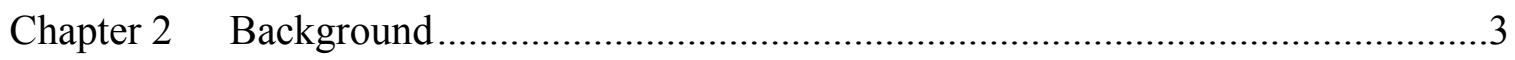

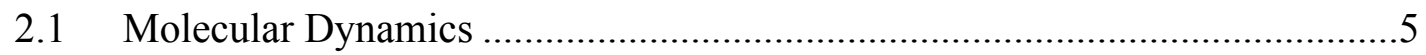

2.1.1 OPLS - All Atom Force Field Parameters .........................................5

2.2 Graphene/Epoxy Composites .....................................................................

Chapter 3 MD modeling: Pure Cycloaliphatic Epoxy.................................................

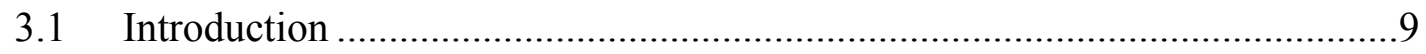

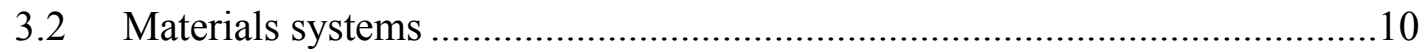

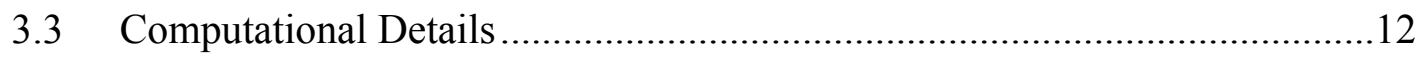

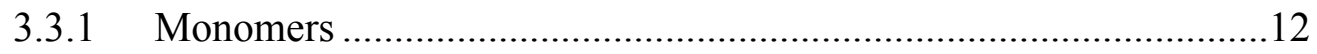

3.3.1.1 Crosslinking Process .......................................................14

3.3.1.2 Equilibration .............................................................. 17

3.3.2 Deformation Models …………………………............................18

3.3.3 Thermomechanical Models.............................................................20

3.3.3.1 Glass-Transition Temperature .........................................20

3.3.3.2 Coefficient of Linear Thermal Expansion .......................22

3.3.4 Thermal Conductivity Models ……………………………...........23 
3.3.4.1 Non-equilibrium Molecular Dynamics .........................23

3.3.4.2 Equilibrium Molecular Dynamics................................25

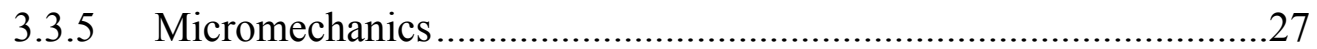

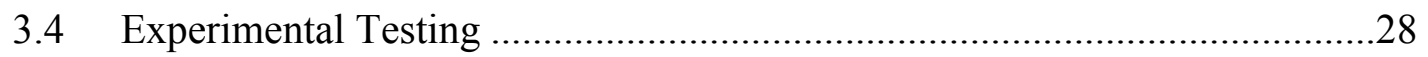

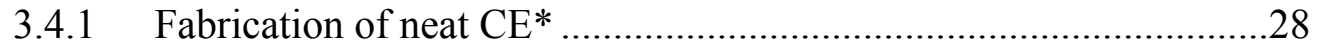

3.4.2 Tensile Testing *......................................................................28

3.4.3 DMA testing for glass-transition temperature* .............................28

3.4.4 Thermal Conductivity Testing* ..................................................29

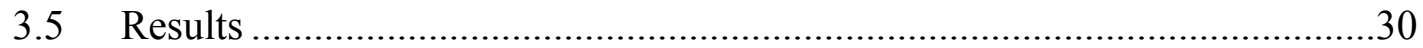

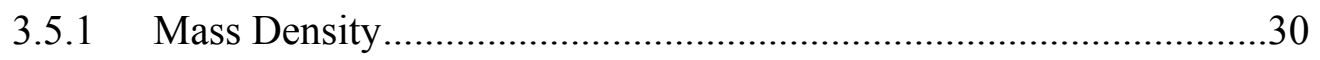

3.5.2 Mechanical Properties................................................................ 30

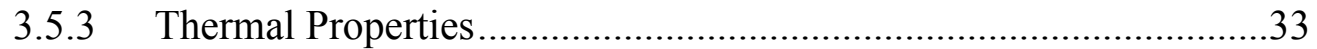

3.5.3.1 Glass-Transition Temperature .............................................

3.5.3.2 Coefficient of Linear Thermal Expansion .......................34

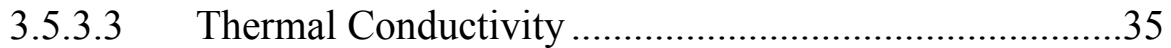

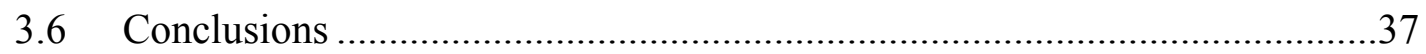

Chapter 4 Multiscale Modeling: Thermal Conductivity of Pristine Graphene Nanoplatelet/Carbon Fiber/Cycloaliphatic Epoxy Hybrid Composites...........................38

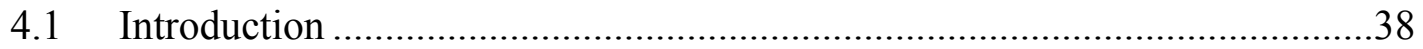

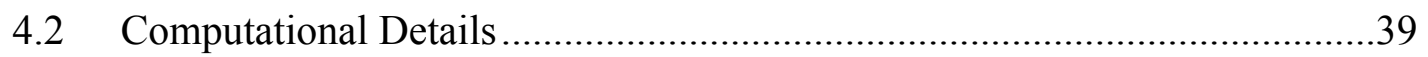

4.2.1 Molecular Modeling.................................................................40

4.2.1.1 GRAPHENE MD MODEL .........................................41

4.2.1.2 NEAT CE AND GNP/CE MODELS .........................41

4.2.1.3 Thermal conductivity by EMD ..................................45

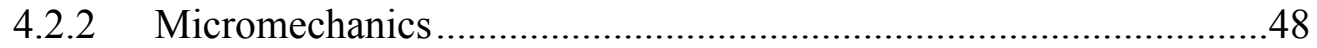

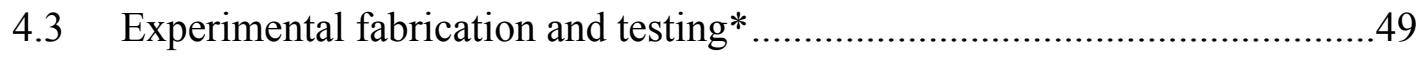

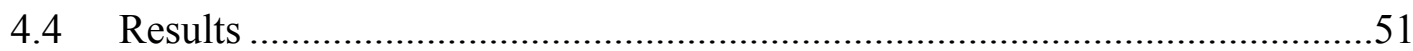


4.5 Conclusions

Chapter 5 Multiscale Modeling: Thermal Conductivity of Carboxyl ( $-\mathrm{COOH}$ ) Functionalized Graphene Nanoplatelet/Cycloaliphatic Epoxy Nanocomposite.....

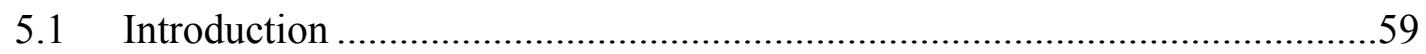

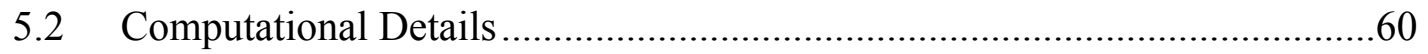

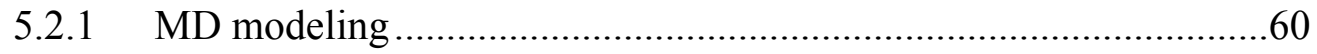

5.2.1.1 Functionalized Graphene MD Model ...........................60

5.2.1.2 fGNP/CE nanocomposites MD Model ........................63

5.2.1.3 Thermal conductivity by EMD ..................................66

5.2.2 Micromechanics ..................................................................6

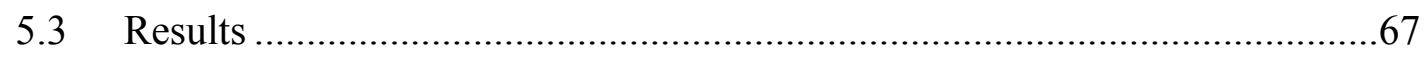

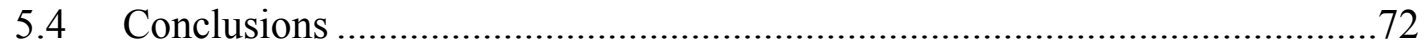

Chapter 6 Multiscale Modeling: Coefficient of Linear Thermal Expansion of Carbon Fiber/Graphene Nanoplatelet/EPON862 Hybrid Composites ......................................73

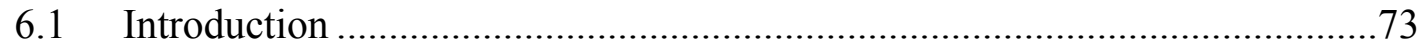

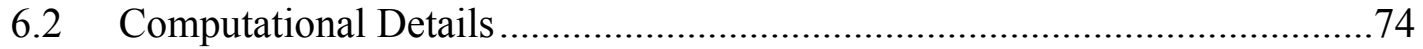

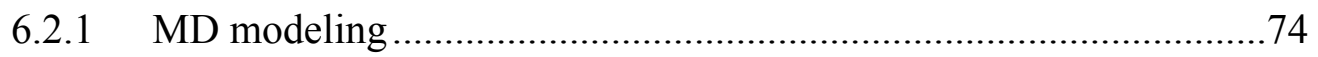

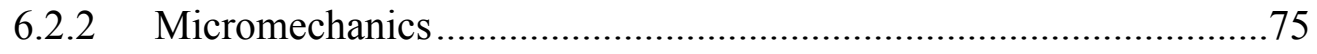

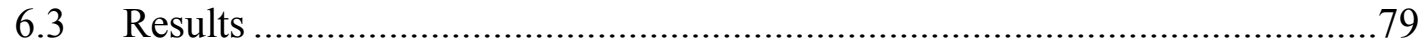

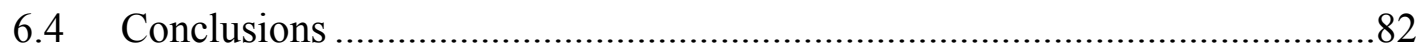

Chapter 7 Improvement of Micromechanics Calculation including GNP Aspect Ratio83

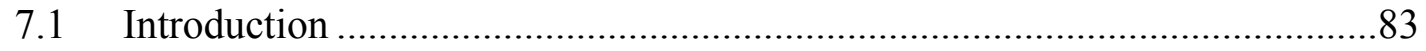

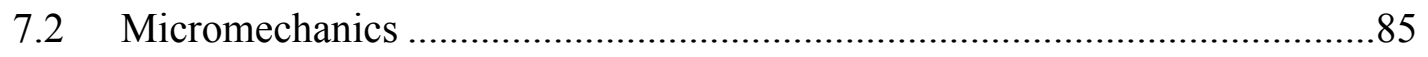

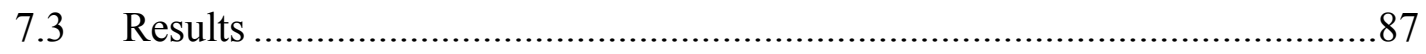


7.4 Conclusions

Chapter 8 Recommendations for Future Work .....................................................92

8.1 Mesoscale Modeling.......................................................................... 92

8.2 The Glass-Transition Temperature on GNP/CE Composites........................92

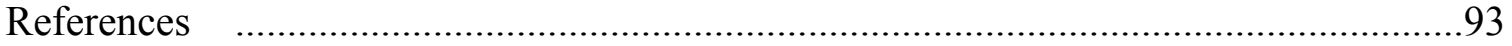

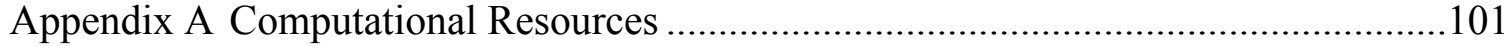

A.1 HPC specification (Michigan Tech) ........................................................... 101

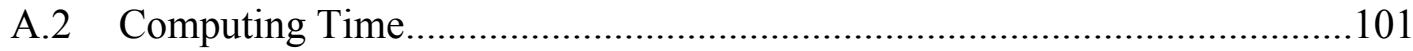

A.2.1 Neat Cycloaliphatic Epoxy .........................................................101

A.2.2 Graphene Nanoplatelet/Cycloaliphatic Epoxy.............................102

A.2.3 Functionalized Graphene Nanoplatelet/Cycloaliphatic Epoxy .......102

A.2.4 CLTE of GNP/EPON862 ......................................................102

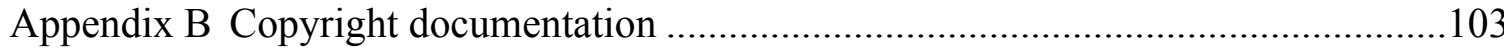

B.1 Copyright of Figure 1, Figure 2, and Figure 3 ...................................... 103

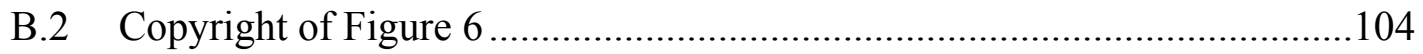

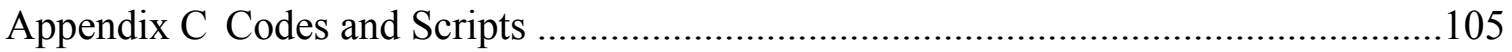

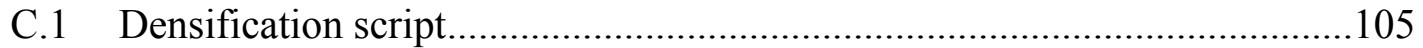

C.2 Crosslink Scripts Package for pure CE .................................................. 108

C.2.1 Initial crosslink script (LAMMPS) .........................................108

C.2.2 Angle and dihedral-updating script (Python)...............................109

C.2.3 Relaxing script(LAMMPS) ................................................. 110

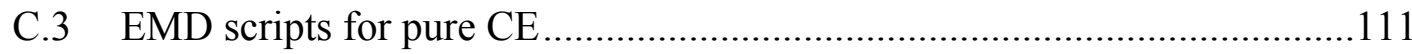




\section{LIST OF FIGURES}

Figure 1 The cross sections of ACSR (left) and ACCC (right), (See Appendix B for

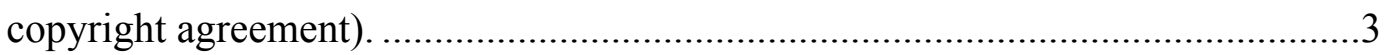

Figure 2 Test data of cable sag as a function of temperature, (See Appendix B for copyright

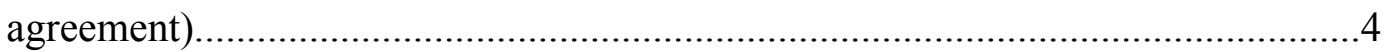

Figure 3 The comparison of the sag between ACSR and ACCC, (See Appendix B for

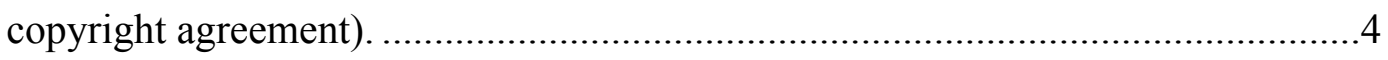

Figure 4 The graphic representations of (a) bond stretching, (b) angle bending, and (c)

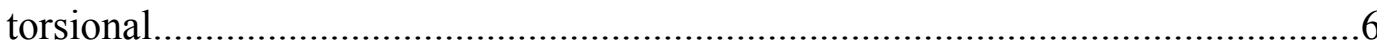

Figure 5 The graphic representation of $\mathrm{VdW}$ interaction as the non-bonded contribution. 7

Figure 6 The visual schematic of thermal interface material showing the difficulty of heat dissipation from heat source. Reprinted with permission from [22]. Copyright 2012 American Chemical Society. (See Appendix B for copyright agreement).............8

Figure 7 Molecular structure of EEC (left) and ACA (right). ....................................11

Figure 8 Esterification reaction between carboxyl and epoxide groups........................11

Figure 9 Cyclic anhydride ring opening reaction between hydroxyl group and anhydride ring .11

Figure 10 Molecular models of EEC (left) and ACA (right). Red: oxygen, black: carbon,

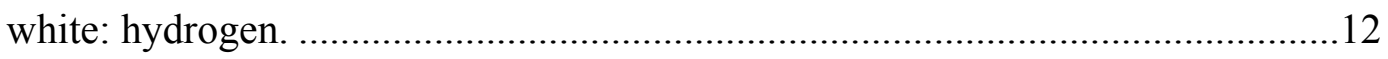

Figure 11 Molecular structure and molecular model of modified ACA. Red: oxygen, black: carbon, white: hydrogen 
Figure 12 Density profiles of $80 \%$ crosslink cubic EEC/ACA model after equilibration along $\mathrm{x}, \mathrm{y}$, and $\mathrm{z}$ direction. .18

Figure 13 A representative crosslinked cubic model of EEC/ACA after equilibration.....18

Figure 14 A representative simulated stress/strain plot of EEC/ACA. .19

Figure 15 A representative Poisson's ratio from the slope between transverse and axial strain. 20

Figure 16 Plot of mass density vs temperature for determining glass-transition temperature for a EEC/ACA model of $80 \%$ crosslink density. .21

Figure 17 Plot of simulation box volume as a polynomial function of temperature from a representative $80 \%$ crosslinked cubic model with the polynomial trend line (solid line) and tangent line (dashed line) at $27^{\circ} \mathrm{C}$.

Figure 18 A representative prism-shaped model for determining the thermal conductivity using NEMD. .24

Figure 19 Simulation box including fixed slaps (grey), $350 \mathrm{~K}$ slab (red), $250 \mathrm{~K}$ slab (blue), and middle slabs (white). .24

Figure 20 Representative plot of the normalized HCACF and thermal conductivity of the CE system as a function of autocorrelation time $(t c)$. .26

Figure 21 Plot of Young's modulus as a function of crosslink density compared with experiment [76]. 31

Figure 22 Predicted axial and transverse elastic modulus as the function of carbon fiber volume fraction. Experimental values of carbon fiber/EPON862 composites are obtained from Kilmek-McDonald [80]. 32 
Figure 23 Predicted glass-transition temperatures of cured epoxy models as a function of crosslink density. Experimentally obtained value (with unknown crosslink density) is overlaid on the data.

Figure 24 Plot of predicted CLTE as a function of crosslink density at $300 \mathrm{~K}$. Literature (experiment) values of unknown crosslink density are overlaid and predicted values from literature are also included. .34

Figure 25 Plot of NEMD and EMD thermal conductivities as a function of crosslink density compared with experiments (Blue region [87-93] and orange line [76]). .35

Figure 26 Plot of thermal conductivity as a function of carbon fiber volume fraction parallel (circle) and transverse (triangle) to the fiber direction. Experimental values taken

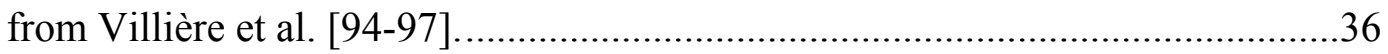

Figure 27. Schematic of multiscale modeling. Red: oxygen, black: carbon, white: hydrogen. .40

Figure 28. Combining of EEC, ACA, modified ACA and GNP into a long simulation box. Then, compressing to the densified model. Black, red, and white color spheres represent carbon, oxygen, hydrogen atoms, respectively. .43

Figure 29 Representative crosslinked models of 1, 2, 3, and 4-layer GNP/CE after equilibration. 44

Figure 30 Normalized heat flux autocorrelation function for 1-layer GNP/CE system. ...46 Figure 31. Normalized heat flux autocorrelation function for 2-layer GNP/CE system. ..46 Figure 32. Normalized heat flux autocorrelation function for 3-layer GNP/CE system. ..47 Figure 33. Normalized heat flux autocorrelation function for 4-layer GNP/CE system...47 
Figure 34. Plot of density profiles of the equilibrated models of 1-layer GNP/CE system.

Figure 35. Plot of density profiles of the equilibrated models of 2-layer GPN/CE system.

Figure 36. Plot of density profiles of the equilibrated models of 3-layer GNP/CE system.

Figure 37. Plot of density profiles of the equilibrated models of 4-layer GNP/CE system.

Figure 38. Plot of normalized thermal conductivity of randomized GNP/CE models as function of GNP mass fraction compared with experiments $[11,14,17,28]$......54

Figure 39. Plot of normalized axial thermal conductivity of GNP/CF/CE hybrid composites models. .56

Figure 40. Plot of normalized transverse thermal conductivity of $\mathrm{GNP} / \mathrm{CF} / \mathrm{CE}$ hybrid composites models compared with experiments [13, 16, 18, 31].

Figure 41 A single-layer GNP model for preparing fGNP model.

Figure 42 The molecular structures of carboxyl groups for placing above (left) and below (right) GNP, respectively. Red: oxygen, gray: carbon, white: hydrogen. 61

Figure 43 Representative pre-functionalized GNP and carboxyl groups models for 55fGNP/CE (left) and 10-10GNP/CE (right) systems.

Figure 44 Molecular structure of 5-5fGNP. .62

Figure 45 Molecular structure of 10-10fGNP. .62

Figure 46 The ratio between defected carbon $\left(\mathrm{SP}^{3}\right)$ and GNP carbon $\left(\mathrm{SP}^{2}\right)$ on $\mathrm{fGNP} . . . .63$ 
Figure 47 Representative crosslinked model of 5-5fGNP/CE after equilibration. .65

Figure 48 Representative crosslinked model of 10-10fGNP/CE after equilibration. .66

Figure 49 The plot of density profiles of 5-5fGNP/CE models along $\mathrm{z}$ axis. .68

Figure 50 The plot of density profiles of 10-10fGNP/CE models along z axis.

Figure 51 The $3^{\text {rd }}$ crosslinked model of 10-10fGNP/CE. 70

Figure 52 The cluster of functional groups shown on the top (left) and bottom (right) surfaces of fGNP of the $3^{\text {rd }} 10-10 \mathrm{fGNP} / \mathrm{CE}$ model.

Figure 53 The plot of normalized thermal conductivity of functionalized and pristine GNP/CE as the function of GNP mass fraction. .71

Figure 54 The representative simple cubic packing RUC. .77

Figure 55 The plot of normalized CLTEs of GNP/EPON862 models as the function of GNP volume fraction compared with the experimental values [112].

Figure 56 The plot of coefficient of thermal expansion along fiber direction as the function of GNP volume fraction. .80

Figure 57 The plot of coefficient of transverse thermal expansion as the function of GNP volume fraction. .81

Figure 58 The dimensions of GNP and GNP/CE simulation box in RUC. .85

Figure 59 The predicted normalized thermal conductivity of single-layered GNP/CE model with different GNP aspect ratios as the function of GNP mass fraction. .88

Figure 60 The predicted normalized thermal conductivity of 4-layered GNP/CE model with different GNP aspect ratios as the function of GNP mass fraction. .88 
Figure 61 Plot of normalized thermal conductivities of 2-layer (Aspect ratio 704) GNP/CE models as the function of GNP volume fraction and the experimental values from

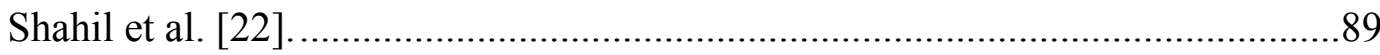

Figure 62 Plot of normalized thermal conductivities of 4-layer (Aspect ratio 200) GNP/CE models as the function of GNP volume fraction and the experimental values from Yu et al. [29]. .90

Figure 63 Plot of normalized thermal conductivities of 1-layer (Aspect ratio 2000) GNP/CE models as the function of GNP volume fraction and the experimental values from Wang et al. [112]. .91 


\section{LIST OF TABLES}

Table 1 The number of molecules and atoms of cubic and prism simulation boxes.... .13

Table 2 The length of the cubic and prism-shape simulation boxes during the densification.

Table 3 The crosslink densities and mass densities of models and experiments. .17

Table 4 Materials properties of neat $\mathrm{CE}$ and carbon fiber.

Table 5 The average elastic properties of EEC/ACA from MD .....................................30

Table 6 The factional coordinates of carbon atom in graphene unit cell.

Table 7 The numbers of total atoms and GNP mass fraction of each system. 42

Table 8 The length of the simulation boxes after the densification. 42

Table 9 The length ratio of the cubic and prism-shape simulation boxes during the densification.

Table 10 Crosslink percentage, mass density, and thermal conductivity of each system. 44

Table 11 Thermal conductivity of neat ce and $4 \mathrm{wt} \%$ gnp/ce from experiment. .50

Table 12 Density, GNP volume fraction, and thermal conductivity of fGNP/CE, GNP/CE and neat $\mathrm{CE}$ at $300 \mathrm{~K}$.

Table 13 Functionality and crosslink density of fGNP/CE nanocomposites models.

Table 14 Elastic properties of GNP/EPON862 models from Hadden et al. [36]. .73

Table 15 Predicted CLTEs of GNP/EPON862 from MD simulations. .75 
Table 16 The randomized elastic moduli and Poisson's ratios of GNP/EPON862 MD

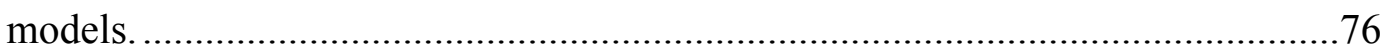

Table 17 Elastic properties and CLTEs of carbon fiber. ............................................... 78

Table 18 The characteristics of graphitic-based filler in graphene/polymer composites studied thermal conductivity from literature........................................................84

Table 19 The GNP thickness and simulation box length of each system.......................86

Table 20 The characteristics of graphene fillers in experimental specimens [22, 29,

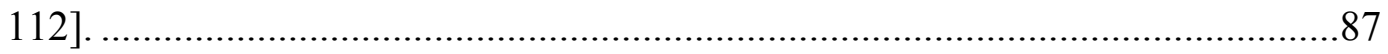




\section{PREFACE}

Dr. Gregory Odegard advised the direction for the course of this work, and reviewed and corrected the portions of manuscript. Dr. Matthew Radue corrected the writing in Chapter 3 and Chapter 4. Julie Tomasi, Dr. Danielle Klimek-McDonald, Dr. Julia King did the experimental testing in Chapter 3 and Chapter 4. 


\section{ACKNOWLEDGEMENTS}

First, I would like to express my sincere thanks to Dr. Gregory Odegard for advising my $\mathrm{PhD}$ study and life. Dr. Odegard provided many opportunities, especially for working in this research project and corrected my research writing.

I really appreciate the help form Dr. Matthew Radue for answering all my questions and having helpful discussions. Matt gave many great ideas in research and been a great officemate.

For providing experimental information, I want to thank Julie Tomasi, Dr. Danielle Klimek-McDonald, and Dr. Julia King. For helping me all about scripting and HPC problems, I would to thank Dr. Gowtham S. for quick response and the helps.

Thanks to the members in this research group; Will Pisani for inviting me to your wedding and giving me the script for MAC/GMC, Hashim Al Mahmud for having good discussions, to Prathamesh Deshpande for inviting to your wedding, to Cameron Hadden for giving me

the EPON862 data files, to Oladeji (Deji) Fadayomi, Sagar Patil, and Muhammed Imam for fulfilling this group to be an awesome research group.

I would like to thank my committees, Dr. Julia King, Dr. Ravi Pandey and Dr. Gowtham S. for their contributions.

This research was funded by the NSF I/UCRC on Novel High Voltage/Temperature Materials and Structures (Grant IIP-1362040), and the Richard and Elizabeth Henes Endowment at Michigan Tech. I have been also supported by Thai government scholarship. I would thank to Dr. Pramote Dechaumpi for influencing me to obtain this scholarship.

Thanks to Thai students for being warm and kind community, especially to Dr. Pichai \& Dr. Rajanee Sripaipan for taking care of us and being a kind doctors, to Nook, Big, Kwang, Ploy, Ple, Puree, Gao, Pon, Bee, Karn, Ming, Benz, Wa, Boat, X, Lek, Gundam, Bo, Pond, 
Tae, Poom, May, Pure, Onn, Amy, Ake, and Ochin for having a good time in Houghton, together.

I also would like thank to my family, especially my parents who sacrifice for me and my brothers. Thanks to my three younger brothers; Golf, Tee, and Jay for supporting my decision. Finally, I would like to thank my love "Kae" who has believed in me and been waiting for me for more than five years. 


\section{ABSTRACT}

The thermal property of epoxy as the binder in the Carbon Fiber (CF) composites, especially thermal conductivity is important to achieve the advance technology and to improve the performance of materials. Multiscale modeling including molecular dynamic (MD) modeling and micromechanical modeling is used to study the properties of neat Cycloaliphatic Epoxies (CE) and Graphene nanoplatelet (GNP)/CE with and without covalent functionalization.

The thermal properties (glass-transition temperature, thermal expansion coefficient, and thermal conductivity) and mechanical properties of CE system are investigated by MD modeling using OPLS-All Atom force field. A unique crosslinking technique is developed to achieve the cured $\mathrm{CE}$ models which has the complex curing mechanism. The thermal conductivity and elastic modulus of $\mathrm{CF} / \mathrm{CE}$ models are further calculated by using micromechanical modeling. The results are validated with the experiments which are in good agreement.

GNP/CE nanocomposites models are established by MD with four different levels of GNP dispersion, namely, 1, 2, 3, and 4 layer(s) of graphene. The thermal conductivities of GNP/CE nanocomposites models are determined by Equilibrium MD (EMD) method. The thermal conductivities are randomized by arithmetic average and varied GNP volume fractions using micromechanics. The resultant thermal conductivities increase with the GNP volume fraction and the better dispersion which compared well with experiments. The 1-layered GNP/CE (perfectly dispersed) model gives the highest thermal conductivity.

The covalently functionalized GNP (fGNP)/CE models are created by functionalizing carboxyl groups onto the single-layered GNP surfaces by MD modeling. The similar method for the pristine GNP/CE models is applied to obtain the effective thermal conductivities fGNP/CE composites. The predicted values suggest that the thermal 
conductivity decreases with increased functionalization on GNP due to the GNP defect. However, the thermal conductivities of fGNP/CE models are higher than the thermal conductivities of 2, 3, and 4-layer GNP/CE models which the experiment found that the functionalization improves the dispersion.

The coefficients of linear thermal expansion (CLTE) of GNP/EPON862 system are studied with the similar work flow which the results show the improvement of CLTE regarding to GNP dispersion. Finally, the GNP aspect ratio is included to improve the micromechanical modeling for thermal conductivity. 


\section{CHAPTER 1 INTRODUCTION}

Carbon fiber/Epoxy composite is an advance material used as the structural component such as aerospace, automotive, high-voltage cable, and sport equipment. This material is very strong, light-weight, chemically resistant, low thermal expansion along the fiber direction. Carbon fiber composite can replace the conventional materials to reduce the weight of product and to improve the chemical resistant. For the vehicle segment, the lighter vehicle uses less fuel which can save the operating cost. For the high-voltage cable segment, the carbon fiber composite core with cycloaliphatic epoxy (CE) in cable can mitigate the thermal sag of cable. This improvement can reduce the chance of accident compared to the conventional cable which elongates and sags at the higher temperature. The carbon fiber composite has been successfully implemented to fabricate the product in the manufacturing scale.

Nevertheless, the carbon fiber composite hardly handles the load transfer in transverse (perpendicular to fiber) direction. The cracks are usually initiated at the interface between epoxy matrix and fiber or in the matrix by itself. There are several researchers who study and improve the mechanical properties in transverse direction. Moreover, the material degradation potentially occurs during the operation due to low thermal conductivity of epoxy matrix. This is because the heat is scarcely dissipated away from the hotspots or the heat source.

To improve the properties of the epoxy matrix as the binder between carbon fibers, the micro particles or nanoparticles are considered to add into the matrix. There are several kind of particles: silicon, metals, carbon black, carbon nanotube, graphene nanoplatelet (GNP), talc, and rubber. Each particle has specifically ability to enhance either thermal or mechanical properties. The mass fraction of these nanoparticles in the composite was studied and some of them showed that the agglomeration of the particle happened at the 
high mass fraction of particle. In this research, the study focuses on the CE which is a unique system with GNP due to its superior materials properties.

The objective of this research is to study and predict thermal and mechanical properties of neat cured CE system using molecular dynamics (MD) modeling. The results of neat CE models are validated with experimental values. After the validation, the thermal conductivities of GNP/CE nanocomposites with different levels of GNP dispersions are studied at molecular level using MD modeling. The bulk thermal conductivities of GNP/CE composites and carbon fiber/GNP/CE hybrid composites are further calculated by using micromechanics. The effect of GNP aspect ratio is also investigated in these calculations. The thermal conductivity of covalently functionalized GNP/CE is predicted how the functionalization affects to this property. Moreover, GNP aspect ratio is additionally investigated in micromechanics. Finally, the thermal expansion coefficients of GNP/EPON862 system are also studied by multiscale modeling. 


\section{CHAPTER 2 BACKGROUND}

The high voltage conductor has been developed for many years because it is very important for transferring electricity to cities, to communities, and to homes. The conventional conductor or the Aluminum Conductor Steel Reinforced (ACSR) has been used for many decades. However, ACSR is not as durable at high temperature which is very dangerous and unreliable due to the thermal expansion of metals. Because the electric transmission needs to be more reliable and more efficient, the Aluminum Conductor Composite Core (ACCC) was developed to replace the ACSR. Both ACSR and ACCC are shown in Figure 1. The outer part of ACSR and ACCC are the aluminum. The core of ACSR is made from steel and the core of ACCC consists of the fiber glass composite and carbon fiber composite which are the yellow and the black color, respectively. Cycloaliphatic epoxy (CE) was used as the binder in carbon fiber composites.

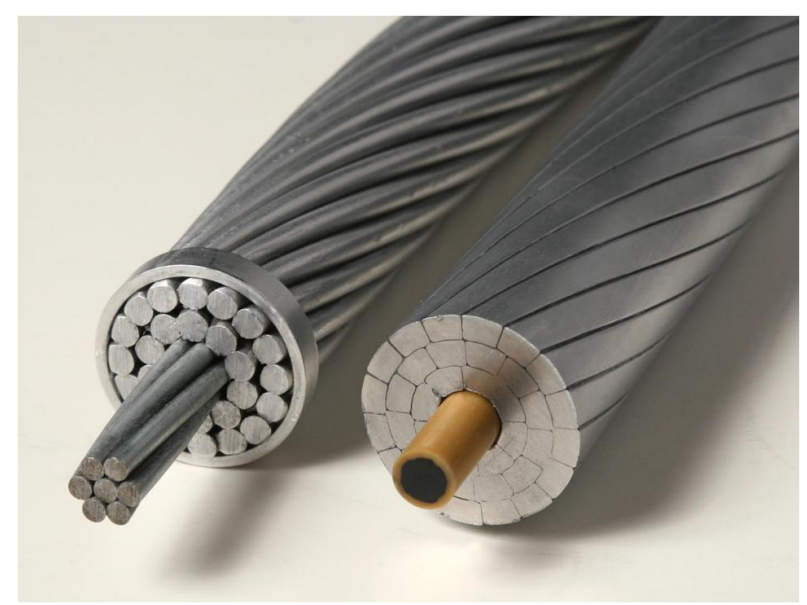

Figure 1 The cross sections of ACSR (left) and ACCC (right), (See Appendix B for copyright agreement).

The thermal expansion of carbon fiber is negative, so the core will not significantly elongate at high temperatures. In Figure 2, the plot shows the cable sags of different conductor's types as the function of temperatures. At $180^{\circ} \mathrm{C}$, the ACCC sags less than 5 
inches, but ACSR sags more than 60 inches. Apparently, ACCC performs much better in this test. Figure 3 shows the comparison of the structural performance between ACSR and ACCC cables which ACCC cables obviously less sag than the ACSR cables.

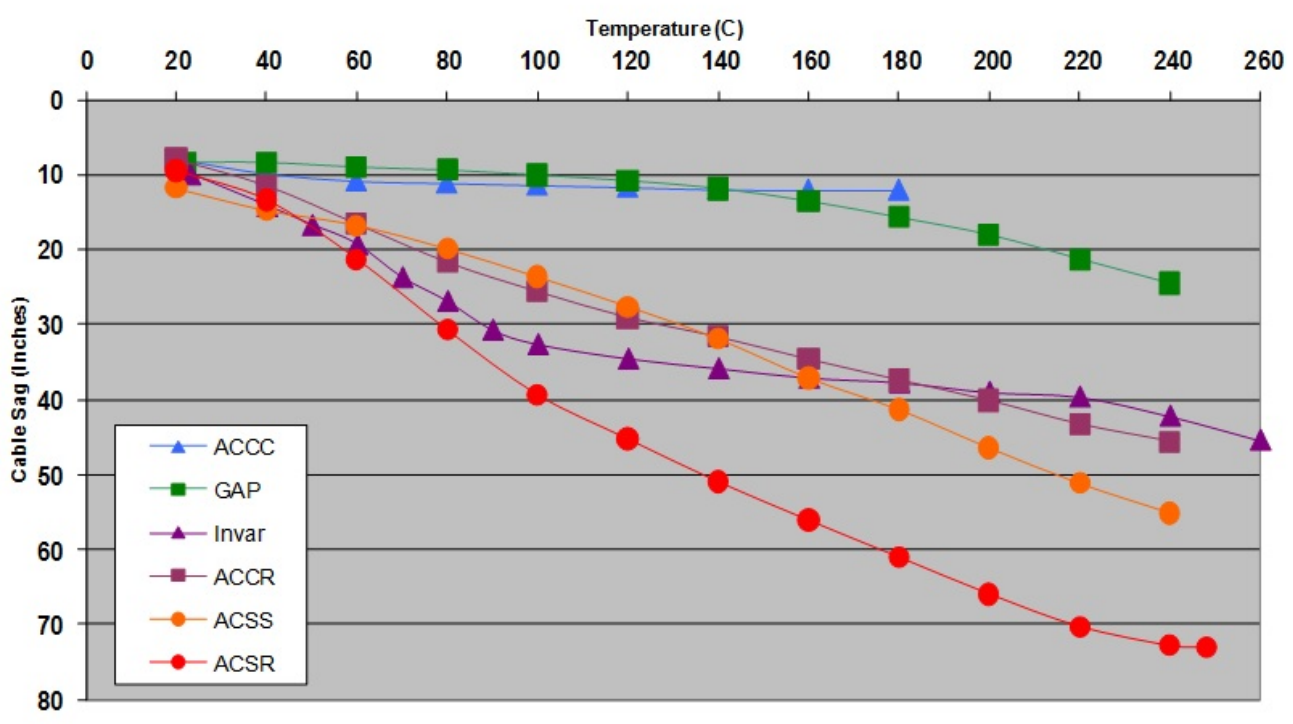

Figure 2 Test data of cable sag as a function of temperature, (See Appendix B for copyright agreement).

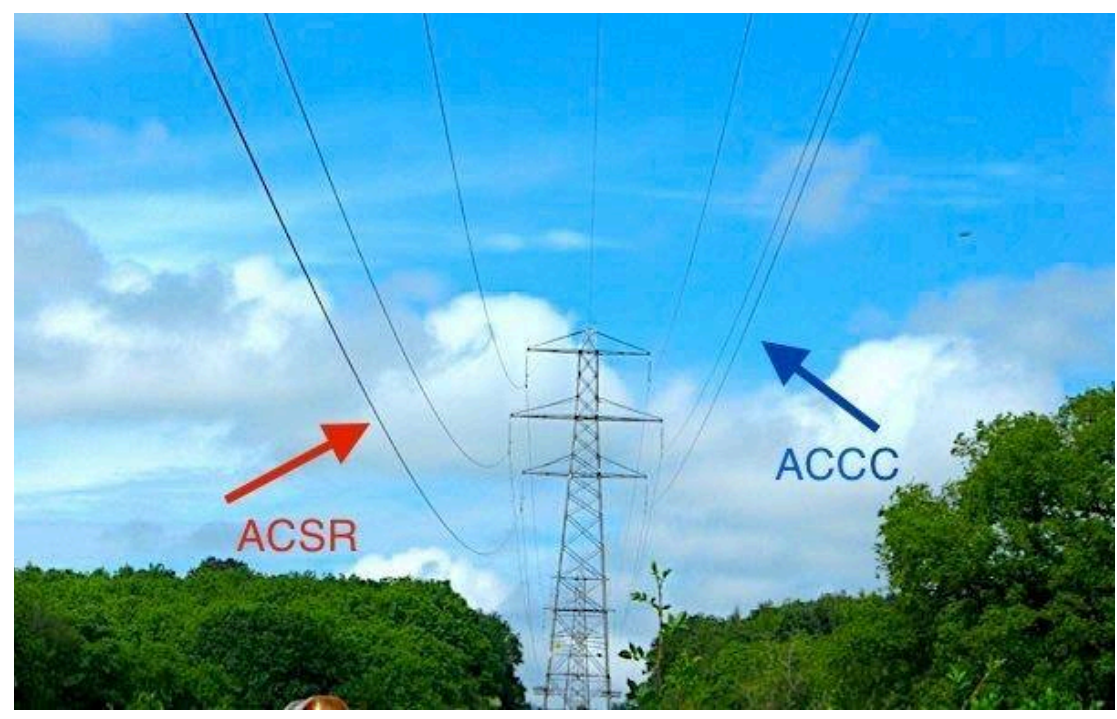

Figure 3 The comparison of the sag between ACSR and ACCC, (See Appendix B for copyright agreement). 


\subsection{Molecular Dynamics}

Molecular dynamics (MD) is a powerful computational tool to simulate the movement of atoms and molecules and to determine thermal and mechanical properties of materials. The movement in the MD simulation is basically based on Newton's law of motion. The simulation determines the position $(\boldsymbol{r})$ and velocity $(\boldsymbol{v})$ vectors of atom $i$ with the time integration using the velocity Verlet algorithm

$$
\begin{aligned}
& \boldsymbol{r}_{i}(t+\Delta t)=\boldsymbol{r}_{i}(t)+\boldsymbol{v}_{i}(t) \Delta t+\frac{1}{2} \boldsymbol{a}_{i}(t) \Delta t^{2} \\
& \boldsymbol{v}_{i}(t+\Delta t)=\boldsymbol{v}_{i}(t)+\frac{\boldsymbol{a}_{i}(t)+\boldsymbol{a}_{i}(t+\Delta t)}{2} \Delta t
\end{aligned}
$$

where $\boldsymbol{a}_{i}$ is the acceleration of atom $i, t$ is the current time, and $\Delta t$ is the time step. The acceleration is calculated by

$$
\boldsymbol{a}_{i}=\frac{\boldsymbol{F}_{i}}{m_{i}}
$$

where $m_{\boldsymbol{i}}$ is mass of atom $i$, and $\boldsymbol{F}_{i}$ is the force vector of atom $i$ obtain from the gradient of the total potential energy $\left(E_{P o t}\right)$ on atom $i$.

$$
\boldsymbol{F}_{i}=\boldsymbol{\nabla}\left(E_{P o t}\right)_{i}
$$

The potential energy is depended on the force field that is applied to the system. More details about that force field is given in the next section.

\subsubsection{OPLS - All Atom Force Field Parameters}

Optimized Potentials for Liquid Simulations (OPLS) - All atom force field [1] was developed by W. L. Jorgensen. This force field is the fix-bonded force field meaning that there is no bond formation and bond scission during the simulation. The potential energies of system are determined from both bonded and non-bonded energies. The bonded 
energetic terms include bond stretching, angle bending, and torsional which the graphic representations of bonded energy term are shown in Figure 4. The bond stretching energy is defined by

$$
E_{\text {bond }}=\sum_{\text {bonds }} K_{r}\left(r-r_{e q}\right)^{2}
$$

where $K_{r}$ and $r_{e q}$ are the stiffness of bond stretching and equilibrium distance constants, $r$ is the current distance between two atoms. The angle bending energy is calculated by

$$
E_{\text {angle }}=\sum_{\text {angles }} K_{\theta}\left(\theta-\theta_{e q}\right)^{2}
$$

where $K_{\theta}$ and $\theta_{e q}$ are the stiffness of angle bending and equilibrium angle constants, $\theta$ is the current angle on three atoms. The torsional or dihedral energy [2] is computed by

$$
\begin{aligned}
E_{\text {torsion }}= & \sum_{\text {dihedrals }}\left[\frac{V_{1}}{2}[1+\cos (\phi)]+\frac{V_{2}}{2}[1-\cos (2 \phi)]\right. \\
& \left.+\frac{V_{3}}{2}[1+\cos (3 \phi)]+\frac{V_{4}}{2}[1-\cos (4 \phi)]\right]
\end{aligned}
$$

where $V_{1}, V_{2}, V_{3}$, and $V_{4}$ are the Fourier coefficients and $\phi$ is the dihedral angle.

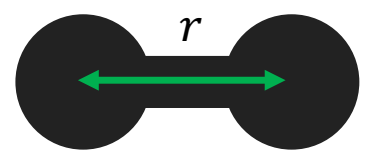

(a)

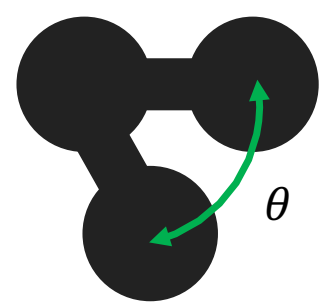

(b)

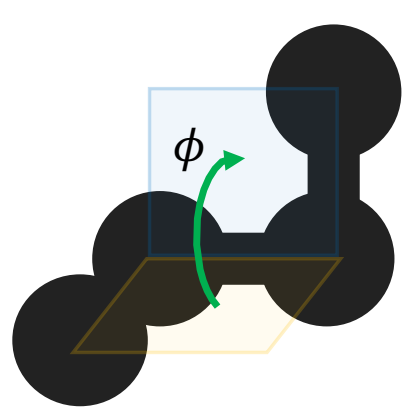

(c)

Figure 4 The graphic representations of (a) bond stretching, (b) angle bending, and (c) torsional. 
Van der Waals (VdW) interaction as the non-bonded energy is based on the 12-6 Lennard - Jones [3] interaction

$$
E_{i j}=4 \varepsilon\left[\left(\frac{\sigma_{i j}}{r_{i j}}\right)^{12}-\left(\frac{\sigma_{i j}}{r_{i j}}\right)^{6}\right]
$$

where $\varepsilon$ is the depth of potential well, $\sigma_{i j}$ is the equilibrium distances between atom $i$ and atom $j$, and $r_{i j}$ the current distances between atom $i$ and atom $j$ shown in Figure 5.

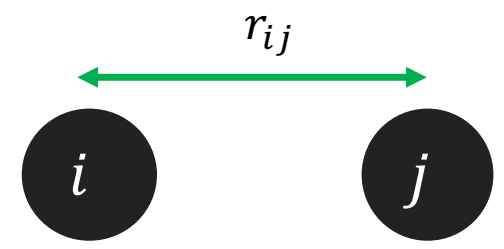

Figure 5 The graphic representation of $\mathrm{VdW}$ interaction as the non-bonded contribution.

The total potential energy combines all energetic contributions shown as following equation.

$$
E_{\text {Pot }}=E_{\text {bond }}+E_{\text {angle }}+E_{\text {torsion }}+E_{V d W}
$$

\subsection{Graphene/Epoxy Composites}

Graphene[4] was successfully exfoliated by repeatedly peeling using "Scotch tape" and first characterized in 2004. Graphene is a single layer of graphite with $\mathrm{sp}^{2}$ carbon atoms. Graphene has the highest thermal conductivity at room temperature which experimentally reported about $5,300 \mathrm{~W} / \mathrm{mK}[5,6]$. This nanomaterial is also the strongest material ever tested which the elastic modulus and tensile strength are equal to $1 \mathrm{TPa}$ and $130 \mathrm{GPa}$ [7], respectively. Because of these promising properties, graphene has been filled into polymer matrix of composites to improve their thermal and mechanical properties.

Many researchers [8-35] experimentally worked on graphene nanoplatelet (GNP)/Polymer composites focused on the mechanical and thermal properties. Thermal conductivities of GNP/epoxy composites were reported in wide range depended on amount of GNP, GNP 
aspect ratio, GNP defect density, and GNP dispersion in epoxy. One of the application that requires improved thermal conductivity is thermal interface materials (TIM) as shown in Figure 6. The interface between the TIM and the die or heat sink has the high temperature different meaning the heat cannot be transferred well in this region.

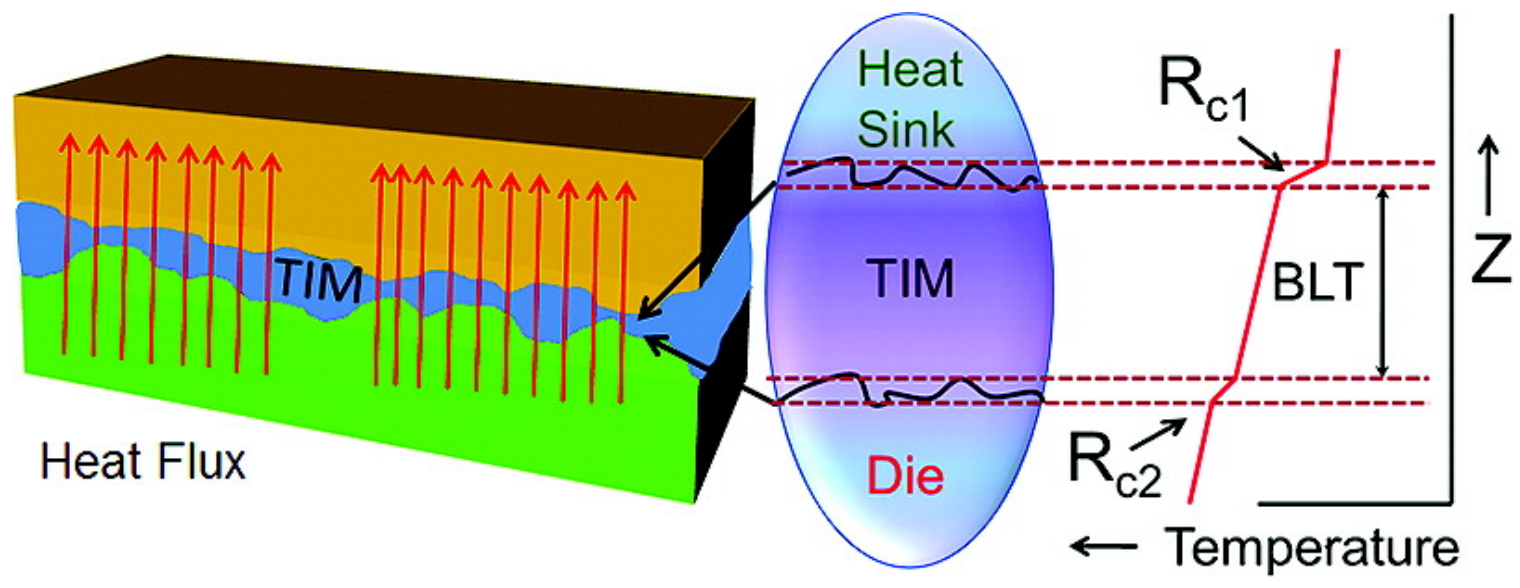

Figure 6 The visual schematic of thermal interface material showing the difficulty of heat dissipation from heat source. Reprinted with permission from [22]. Copyright 2012 American Chemical Society. (See Appendix B for copyright agreement).

The MD modeling was utilized to investigate the characterization and mechanical properties of GNP/EPON862/DETDA system [36, 37] with OPLS - united atom force field. Non-Equilibrium MD (NEMD) was performed to determine the thermal conductivity of GNP/EPON862/TETA system [38-40]. The NEMD method requires a long simulation box which is able to find only thermal conductivity on the long direction. For the GNP/epoxy models, two different models are needed to determine the in-plane and out-ofplane thermal conductivities. To keep the same GNP volume fraction in both models is very challenging. In this research, Equilibrium MD (EMD) approach was selected to determine the thermal conductivity of the nanocomposites. The details of the EMD method is given in Chapter 4. 


\section{CHAPTER 3 MD MODELING: PURE CYCLOALIPHATIC EPOXY}

\subsection{Introduction}

Cycloaliphatic epoxies (CE) are commonly used for structural applications requiring improved resistance to elevated temperatures, UV radiation, and moisture relative to Bisphenol A (DGEBA) and Bisphenol F (DGEBF) Diglycidyl Ether epoxies [26, 41, 42]. Thus, CE composites are particularly important in applications such as high-voltage electrical insulators and Aluminum Conductor Composite Core (ACCC) high-voltage power transmission cables, particularly when they are paired with an anhydride curing agent (ACA) which also demonstrates remarkable environmental resistance. ACCC lines must withstand these harsh environmental conditions in addition to localized heating due to transmission line faults (e.g. failing connections and leakage currents). It is imperative that these lines be designed to mitigate localized heating quickly to reduce local material damage. Thus, the composite core component of the ACCC lines should have an optimal level of thermal conductivity. Also, the thermal expansion coefficient should be optimized to mitigate thermal sag in the lines. Accurate and efficient computational models can greatly facilitate the development of these composite core materials to meet these thermal property requirements.

Over the last decade, molecular dynamics (MD) simulation has become an important tool for the prediction of thermo-mechanical properties of epoxy materials. The thermal and mechanical response of DGEBF [37, 43-52] and DGEBA [51, 53-58] crosslinked epoxy systems has been investigated with numerous MD studies. These epoxy systems have become benchmark materials for MD simulation partially due to their relatively simple crosslinking reaction. For $\mathrm{CE} / \mathrm{ACA}$, the chain reaction mechanism is much more complex. The only known molecular modeling study for CE/ACA is from Komarov et al. [59], who investigated the system using coarse-grained Monte Carlo simulation for crosslinking, 
followed by reversed-mapping to a fully atomistic MD model. Thus, a fully atomistic procedure for modeling the crosslinking of $\mathrm{CE} / \mathrm{ACA}$ resins has not been developed.

The objectives of this study were to (1) develop an all-atom MD-based modeling procedure to accurately and efficiently simulate the crosslinking process of CE/ACA resins and to (2) predict the bulk-level thermal and mechanical properties of carbon fiber-reinforced $\mathrm{CE} / \mathrm{ACA}$ composites for a range of carbon fiber volume fractions using a multiscale modeling approach, which includes the MD modeling for atomic length scales and micromechanics for bulk length scales. The modeling procedure was validated with experimental data determined as described herein and from the literature.

This chapter is structured as follows. First, the details of the polymer material system are provided, including the molecular structures of the resin and hardener and the curing mechanism. Second, details are provided about the multiscale modeling procedure. Third, the experimental methods for measurement of the mass density, elastic properties, glasstransition temperature, and thermal conductivity are described. Finally, the modeling results are discussed with respect to the experimental values.

\subsection{Materials systems}

Epoxy cyclohexyl methyl 3,4 epoxy cyclohexyl carboxylate (EEC) is a CE resin as characterized by the aliphatic carbon rings fused to reactive epoxide groups. For EEC, there are two epoxide groups per molecule as shown in Figure 7. The ACA is 4, 7Methanoisobenzofuran-1, 3-dione, 3a, 7, 7a-tetrahydromethyl which contains a cyclic anhydride ring and bi-cyclic component, also shown in Figure 7. 


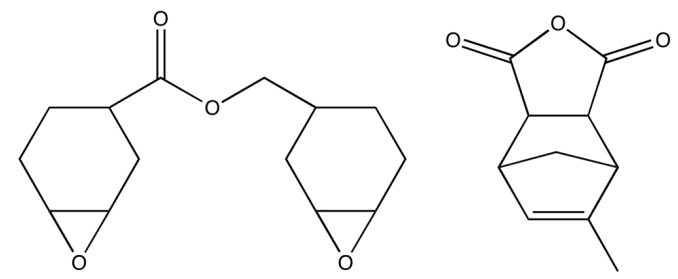

Figure 7 Molecular structure of EEC (left) and ACA (right).

The curing mechanism $[60,61]$ of the EEC/ACA system consists of two main alternating reactions: an esterification reaction and a cyclic anhydride ring opening reaction. Figure 8 shows the esterification reaction between an epoxide group and carboxyl group, which requires a catalyst to start the reaction. This reaction generates an ester chain and hydroxyl group that is necessary for opening the cyclic anhydride ring. Figure 9 shows the cyclic anhydride ring opening reaction. This reaction is initiated by a hydroxyl group from the esterification reaction. A carboxyl group is created and continues again as the reactive group in the esterification reaction. Both reactions continue until all nearby reactive groups are reacted.

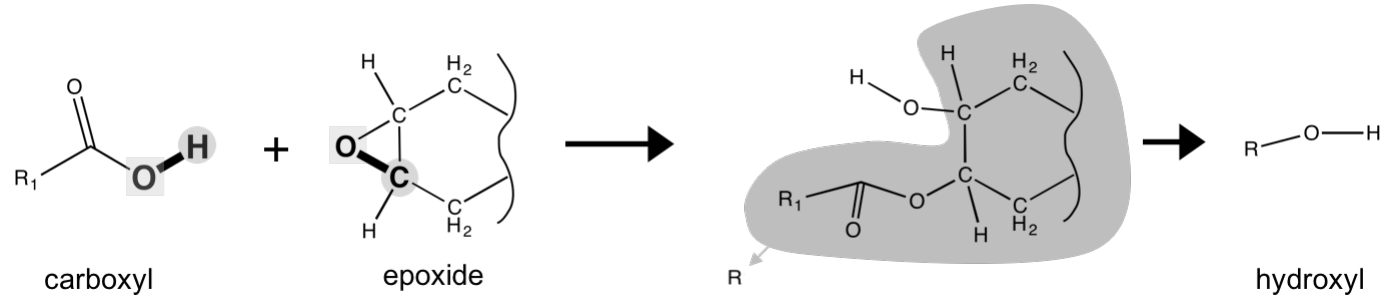

Figure 8 Esterification reaction between carboxyl and epoxide groups.

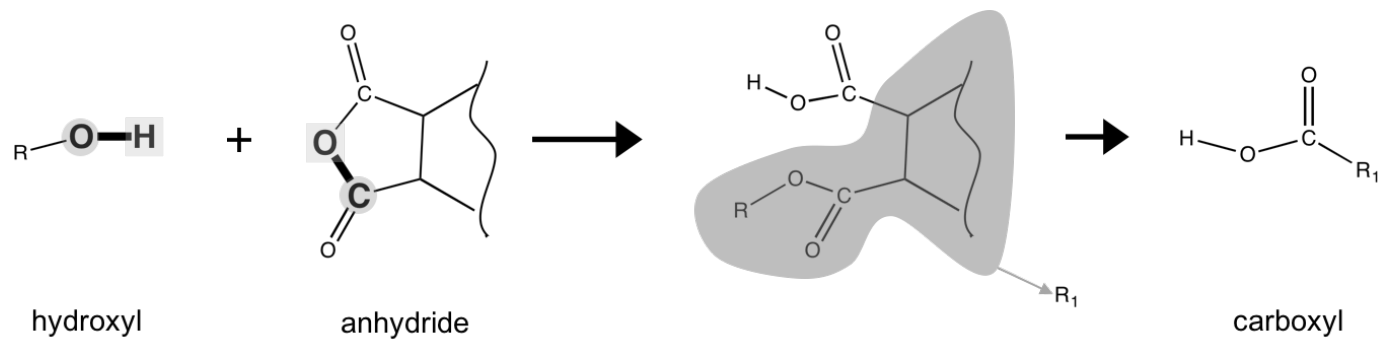

Figure 9 Cyclic anhydride ring opening reaction between hydroxyl group and anhydride ring. 


\subsection{Computational Details}

The multi-scale computational approach employed in this study includes both MD and Micromechanical modeling. MD modeling was used to determine thermal properties of the neat epoxy based on the molecular structure. For the micromechanics modeling, the results from the MD modeling were used as input into a larger-scale Representative Volume Element (RVE) to predict the effective thermal conductivity of carbon fiber/CE composites. All details of the modeling are given in this section.

\subsubsection{Monomers}

All neat epoxy models were simulated at the molecular level using the Large-scale Atomic/Molecular Massively Parallel Simulator (LAMMPS) MD software package [62] with the OPLS - AA (Optimized Potentials for Liquid Simulations - All Atom) force field $[1,63]$. Monomeric structures of EEC and ACA were initially created using ChemBioDraw [64] and subsequently imported into LAMMPS. The force constants associated with bond, angle, dihedral, and van der Waal (vdW) interactions were used. The topology of both monomers were equilibrated using the Conjugated-Gradient method, as implemented in LAMMPS, with a $1 \times 10^{-4}$ energy tolerance. MD simulations were subsequently performed with the NVT ensemble for $1 \mathrm{~ns}$ with $1 \mathrm{fs}$ time steps. In Figure 10, the resulting molecular structures of EEC and ACA monomers were rendered using the Open Visualization Tool (OVITO) software package [65].
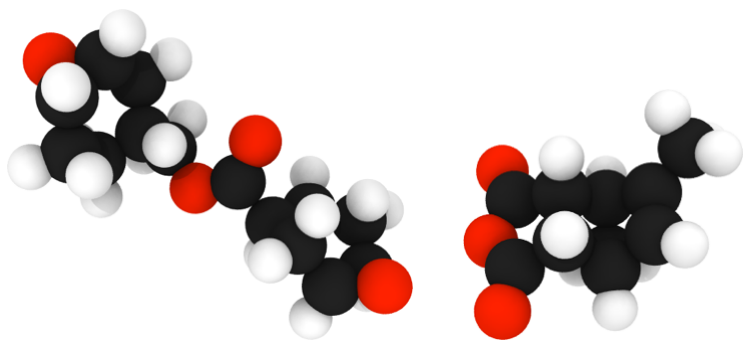

Figure 10 Molecular models of EEC (left) and ACA (right). Red: oxygen, black: carbon, white: hydrogen. 
For the simulated crosslinking, the curing mechanism discussed above required a catalyst to start the chain reaction. A sub-set of the ACA molecules were modified with an ethanol molecule [66], as shown in Figure 11. The cyclic anhydride ring on the ACA molecule was opened with the hydroxyl group on the ethanol molecule. The exposed carboxyl group was therefore available to react with epoxide groups for the esterification reaction (Figure 2).
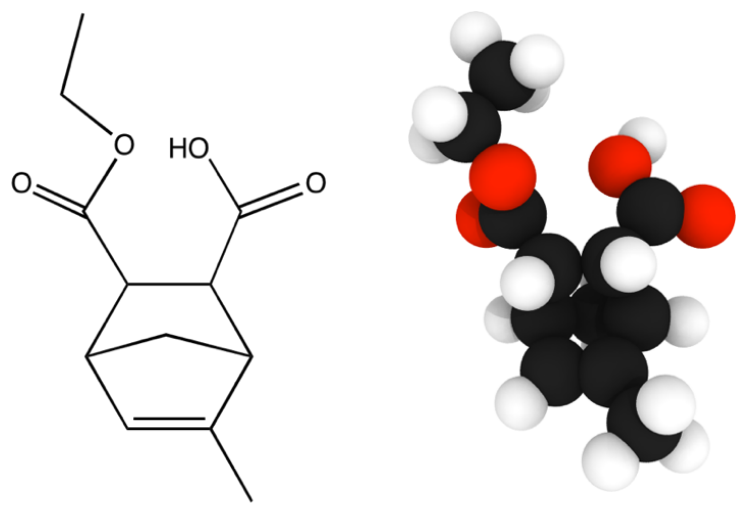

Figure 11 Molecular structure and molecular model of modified ACA. Red: oxygen, black: carbon, white: hydrogen.

Bulk molecular models of the EEC/ACA system were generated using a multi-step process. The molar ratio between the CE resin and ACA was 1:2. The monomers of EEC, ACA, and modified ACA were mixed as shown in Table 1. These monomers were combined in a large MD simulation box. The initial mass density of the simulation box was less than $0.008 \mathrm{~g} / \mathrm{cm}^{3}$ and all boundaries were periodic. For the results discussed herein, all thermal properties were determined from a cubic simulation box, except for the thermal conductivity simulations with direct method (described below), which utilized a prismshaped simulation box.

Table 1 The number of molecules and atoms of cubic and prism simulation boxes.

\begin{tabular}{ccccc}
\hline \multirow{2}{*}{ Shape } & \multicolumn{3}{c}{ Number of molecules } & \multirow{2}{*}{ Number of atoms } \\
\cline { 2 - 4 } & EEC & ACA & Modified ACA & \\
Cube & 84 & 156 & 12 & 7,164 \\
Prism & 126 & 234 & 18 & 10,746 \\
\hline
\end{tabular}


The simulation boxes were alternately subjected to potential energy equilibrations and simulation box size reductions using the "minimize" and "fix deform" commands in LAMMPS, respectively. The simulation boxes were gradually compressed to a density of $1.2 \mathrm{~g} / \mathrm{cm}^{3}$, which is the accepted bulk density of most epoxy resins [67]. The box lengths in along the $\mathrm{x}, \mathrm{y}$, and $\mathrm{z}$ directions were sequentially reduced to their final length by densifying in four different steps as shown in Table 2 over a total simulation time of $4.2 \mathrm{~ns}$ with $1 \mathrm{fs}$ time steps. The density profiles of the densified models were spatially uniform along the $\mathrm{x}, \mathrm{y}$, and $\mathrm{z}$ directions, and thus the MD models were well-equilibrated in the uncrosslinked state.

Table 2 The length of the cubic and prism-shape simulation boxes during the densification.

\begin{tabular}{cccccccc}
\hline \multirow{2}{*}{ Step } & \multicolumn{3}{c}{ Cube $(\AA)$} & \multicolumn{3}{c}{ Prism $(\AA)$} & \multirow{2}{*}{$\begin{array}{c}\text { Time } \\
(\mathrm{ns})\end{array}$} \\
\cline { 2 - 7 } & $\mathrm{x}$ & $\mathrm{y}$ & $\mathrm{z}$ & $\mathrm{x}$ & $\mathrm{y}$ & $\mathrm{z}$ & - \\
\hline 0 & 300.00 & 200.00 & 200.00 & 300.00 & 300.00 & 300.00 & - \\
1 & 41.50 & 41.50 & 41.50 & 26.15 & 26.15 & 156.88 & 0.2 \\
2 & 23.86 & 23.86 & 23.86 & 15.03 & 15.03 & 90.20 & 1.0 \\
3 & 21.79 & 21.79 & 21.79 & 13.73 & 13.73 & 82.36 & 2.0 \\
4 & 20.75 & 20.75 & 20.75 & 13.07 & 13.07 & 78.44 & 1.0 \\
\hline
\end{tabular}

\subsubsection{Crosslinking Process}

The crosslink process was iterative process combining LAMMPS and Python script. For this approach, the crosslink density can be specified which $60 \%, 65 \%, 70 \%, 75 \%, 80 \%$, $85 \%$, and $90 \%$ crosslink percentage of EEC/ACA system were aimed in this work.

Schematic 1 shows the simulated crosslinking process with MD steps labeled as "LAMMPS" and the "Python" labels corresponding to Python scripting designed to delete and create all bonds, angles, and dihedrals. The Python scripts also updated pair coefficient of relative atoms. The labels " 1 " and " 2 " correspond to the esterification reaction (Figure 2) and cyclic anhydride ring opening (Figure 3) reactions, respectively. Following the densification, the data file for the densified model was used for LAMMPS 1 to initially 
create the carbon-oxygen bond in the esterification reaction. The updated data file was subsequently used for the Python 1 step to create and delete all related bonds, angles, and dihedrals. Following this, the data file was run in the LAMMPS 2 step to initially generate the oxygen-hydrogen bond of the cyclic anhydride ring opening reaction and Python 2 updated all information for this reaction in the same manner as Python 1.

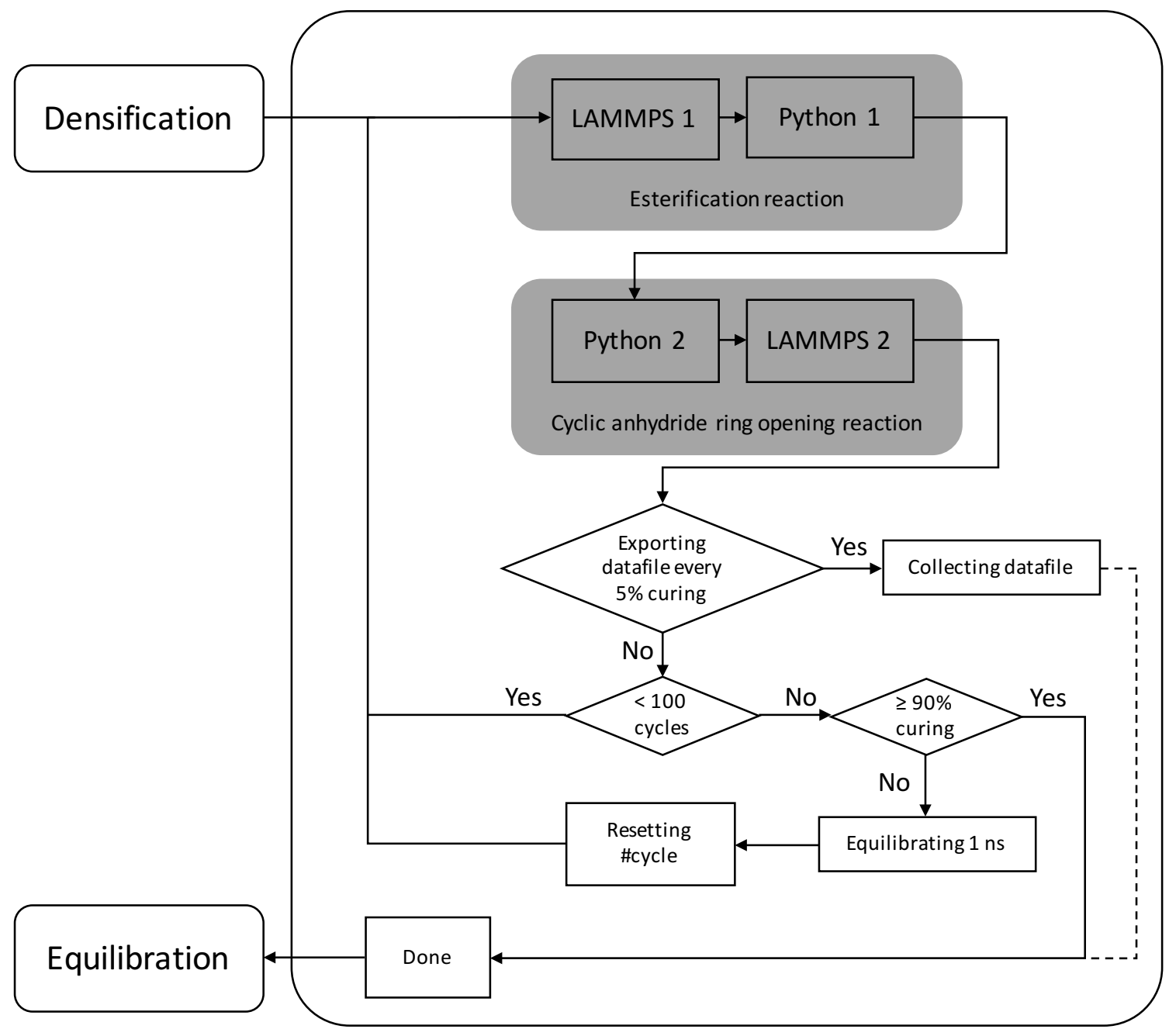

Schematic 1 Crosslinking process of EEC/ACA 
The crosslink percentage was calculated in the Python 2 step by dividing total number of formed ester bonds by the total number of epoxide groups at the beginning of the crosslinking process. The data files which were close to $60 \%, 65 \%, 70 \%, 75 \%, 80 \%, 85 \%$, and $90 \%$ of crosslink percentage were exported to different folders to study the effect of crosslink density on materials properties. After the Python 2 step was completed, the current crosslinking step was complete and the structure file was passed back to LAMMPS 1 for subsequent crosslinking steps. After one hundred cycles, the structure file was equilibrated in the NPT (constant pressure and temperature) ensemble for 1 ns with $1 \mathrm{fs}$ time steps. If the crosslink density was less than $90 \%$, the structure file was imported back into the LAMMPS/Python steps for another 100 cycles. Once the crosslink percentage was equal to or greater than $90 \%$, the crosslinking process was completed and the model was fully equilibrated with the NPT ensemble as mentioned above. The details of LAMMPS 1, Python 1, LAMMPS 2, and Python 2 steps are provided below.

The LAMMPS 1 step initially created the bond between carbon atom and oxygen atom from epoxide and carboxyl groups, respectively, by using 'fix bond/create' command and creating the bond every time step with 0.001 probability within a $7 \AA$ threshold. The NVT ensemble was applied at $300 \mathrm{~K}$, which ran for $200 \mathrm{fs}$ with $0.1 \mathrm{fs}$ time steps. The Python 1 step created the bond between the oxygen and hydrogen atoms from epoxide and carboxyl groups, respectively, which provided the hydroxyl group. The old bonds, which were carbon-oxygen in the epoxide group and oxygen-hydrogen in the carboxyl group, were assigned to be a special bond type for deletion in the LAMMPS 2 step, along with the associated angles and dihedrals.

The LAMMPS 2 step began with generating the bond between the oxygen and hydrogen atoms from cyclic anhydride ring and hydroxyl group, respectively, in the same manner as LAMMPS 1. The Python 2 step established the shorter-distanced bond between the carbon and oxygen atoms from anhydride ring and hydroxyl group, respectively. The shorterdistanced bond can prevent any over-stretch bond. The oxygen-hydrogen and carbonoxygen bonds from the hydroxyl group and anhydride ring, respectively, were assigned for 
deletion in the LAMMPS 1 step. All angles and dihedrals were updated in the same manner as with Python 1. For LAMMPS 1 and LAMMPS 2, the special bond is removed at the beginning of script by using "delete_bond".

\subsubsection{Equilibration}

After the crosslinking process, the models were equilibrated with the NPT ensemble at 1 ATM and $300 \mathrm{~K}$ to allow residual stresses to be minimized. Figure 12 shows representative mass density profiles along each of the Cartesian axes. The relatively small fluctuations in density indicate that the model has minimal localized residual stresses within the simulation box. The mass density was calculated from averaging the last $10 \mathrm{ps}$ of each simulation. The average mass densities and conversion levels are provided in Table 3. Figure 13 shows a representative crosslinked model after equilibrating for $2 \mathrm{~ns}$ with $1 \mathrm{fs}$ time steps. After crosslinking, the models were ready for further simulation to predict the thermal properties.

Table 3 The crosslink densities and mass densities of models and experiments.

\begin{tabular}{cccc}
\hline \multicolumn{2}{c}{ Cube } & \multicolumn{2}{c}{ Prism } \\
\hline Crosslink density & Mass Density $\left(\mathrm{g} / \mathrm{cm}^{3}\right)$ & Crosslink density & Mass Density $\left(\mathrm{g} / \mathrm{cm}^{3}\right)$ \\
\hline $0.60 \pm 0.00$ & $1.211 \pm 0.003$ & $0.60 \pm 0.00$ & $1.209 \pm 0.005$ \\
$0.66 \pm 0.01$ & $1.214 \pm 0.006$ & $0.65 \pm 0.00$ & $1.206 \pm 0.004$ \\
$0.70 \pm 0.00$ & $1.210 \pm 0.004$ & $0.70 \pm 0.00$ & $1.207 \pm 0.005$ \\
$0.75 \pm 0.01$ & $1.204 \pm 0.004$ & $0.75 \pm 0.00$ & $1.204 \pm 0.005$ \\
$0.80 \pm 0.00$ & $1.199 \pm 0.006$ & $0.80 \pm 0.00$ & $1.193 \pm 0.005$ \\
$0.85 \pm 0.00$ & $1.191 \pm 0.006$ & $0.85 \pm 0.00$ & $1.187 \pm 0.005$ \\
$0.90 \pm 0.00$ & $1.183 \pm 0.007$ & $0.90 \pm 0.01$ & $1.187 \pm 0.001$ \\
\hline
\end{tabular}




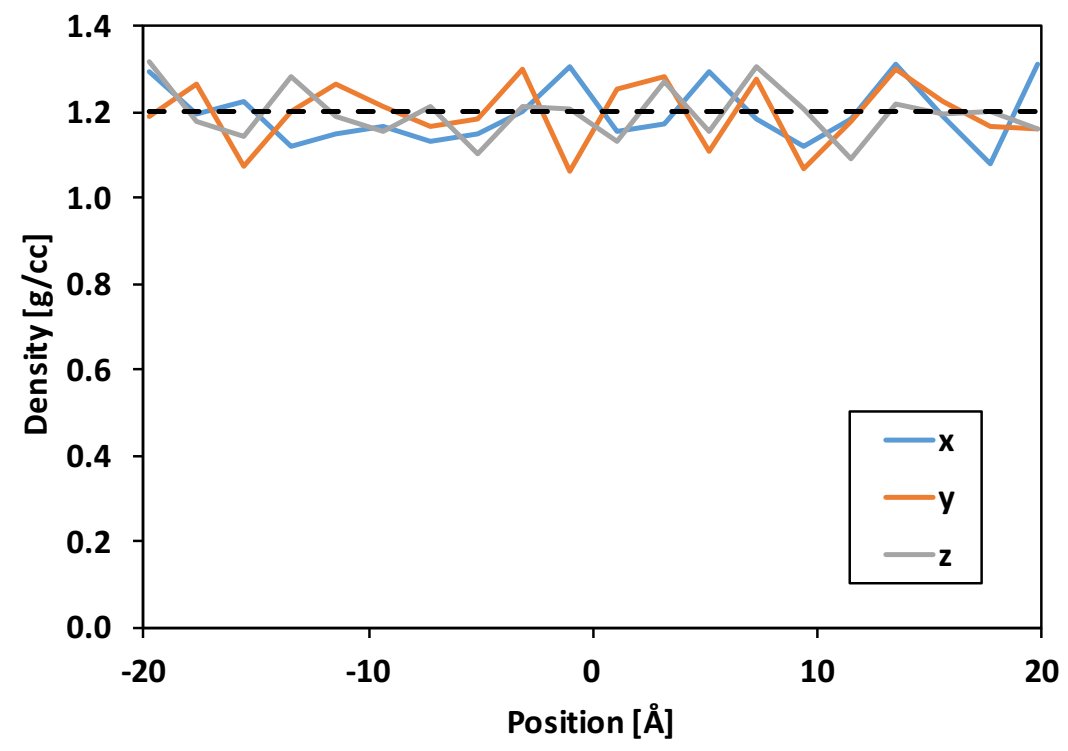

Figure 12 Density profiles of $80 \%$ crosslink cubic EEC/ACA model after equilibration along $\mathrm{x}, \mathrm{y}$, and $\mathrm{z}$ direction.

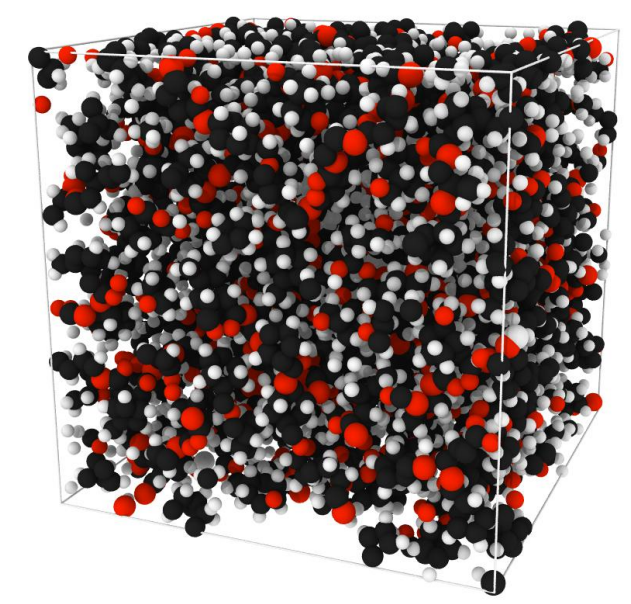

Figure 13 A representative crosslinked cubic model of EEC/ACA after equilibration.

\subsubsection{Deformation Models}

Elastic properties were determined by uniaxial tensile deforming the crosslinked models.

$$
E_{x}=\frac{\sigma_{x}}{\varepsilon_{x x}}
$$


As shown above, the isotropic Young's modulus was calculated from the initial slope of true stress/true strain plot in $\mathrm{x}, \mathrm{y}$, and $\mathrm{z}$ directions and averaged all directions. The MD models were deformed uniaxially using the "fix deform" command in LAMMPS with an engineering strain rate of $1 \times 10^{8} \mathrm{~s}^{-1}$. For a given deformation along a principal direction, the lateral dimensions of the simulation box were allowed to have the natural Poisson's contraction by setting 1 ATM pressure with the NPT ensemble at $300 \mathrm{~K}$. These simulations ran for $2 \mathrm{~ns}$ with $1 \mathrm{fs}$ time steps. During the simulations, all stresses and strains of the models were averaged every 4 ps. Figure 14 shows a representative plot for determining the slope using linear regression on the data that was below a strain of 0.04 . The Poisson's ratio was calculated from negative slope of transverse and axial strain plot shown in Figure 15.

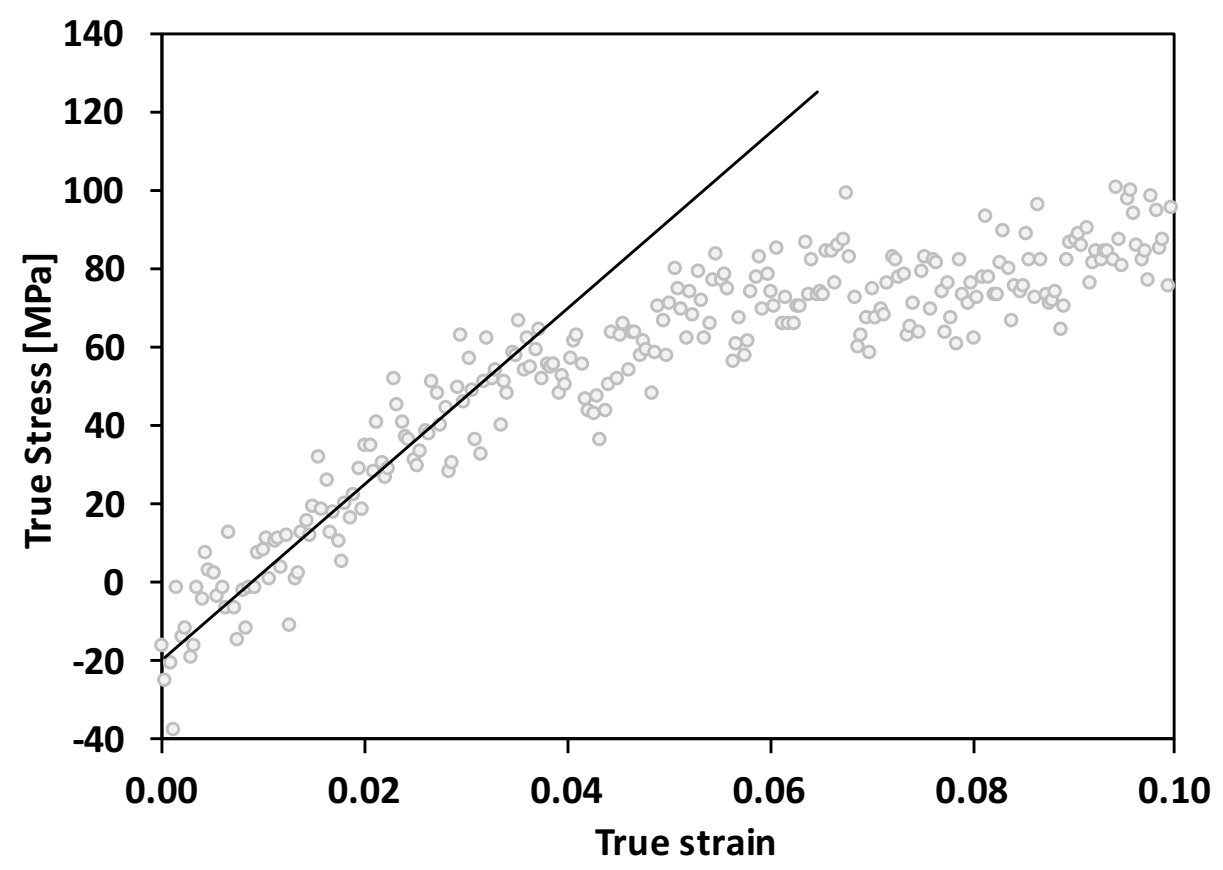

Figure 14 A representative simulated stress/strain plot of EEC/ACA. 


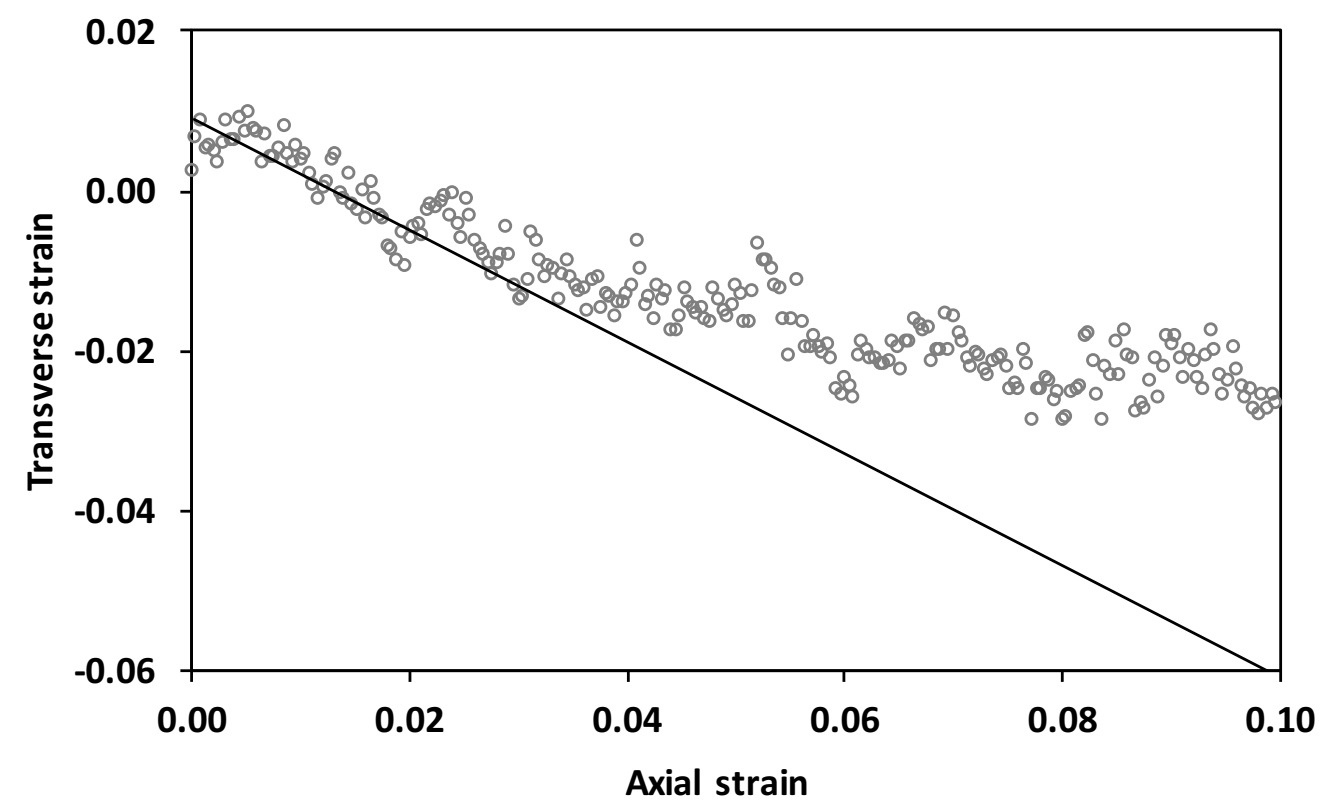

Figure 15 A representative Poisson's ratio from the slope between transverse and axial strain

$$
G_{x y}=\frac{\tau_{x y}}{\gamma_{x y}}
$$

The simulation boxes were also subjected to shearing deformations, and the shear modulus was determined from the initial slope of the shear stress $(\tau)$ and engineering shear strain $(\gamma)$ response using Equation (5). The boxes were deformed using the 'fix deform' command in LAMMPS in the $\mathrm{xy}, \mathrm{xz}$, and yz planes in individual simulations. Each simulation ran for $2 \mathrm{~ns}$ with $1 \mathrm{fs}$ time steps.

\subsubsection{Thermomechanical Models}

\subsubsection{Glass-Transition Temperature}

The glass transition temperature was determined for each crosslink density using the method of Bandyopadhyay et al. [46]. The temperature of the crosslinked cubic EEC/ACA simulation boxes was increased from -100 to $527{ }^{\circ} \mathrm{C}(173-800 \mathrm{~K})$ in the NPT ensemble 
under $1 \mathrm{~atm}$ pressure. The model was gradually heated over $5 \mathrm{~ns}$ with $1 \mathrm{fs}$ time steps. The heating rate was $1.25 \times 10^{11}{ }^{\circ} \mathrm{C} / \mathrm{s}$. The mass densities and temperatures of the MD models were averaged every 2 ps throughout the simulations and plotted as shown in Figure 16 for a representative system. The two linear trend lines were fit using the "segmented" package [68] in the RStudio [69] integrated development environment. The glass-transition temperature was defined as the temperature corresponding to the interception of the trend lines.

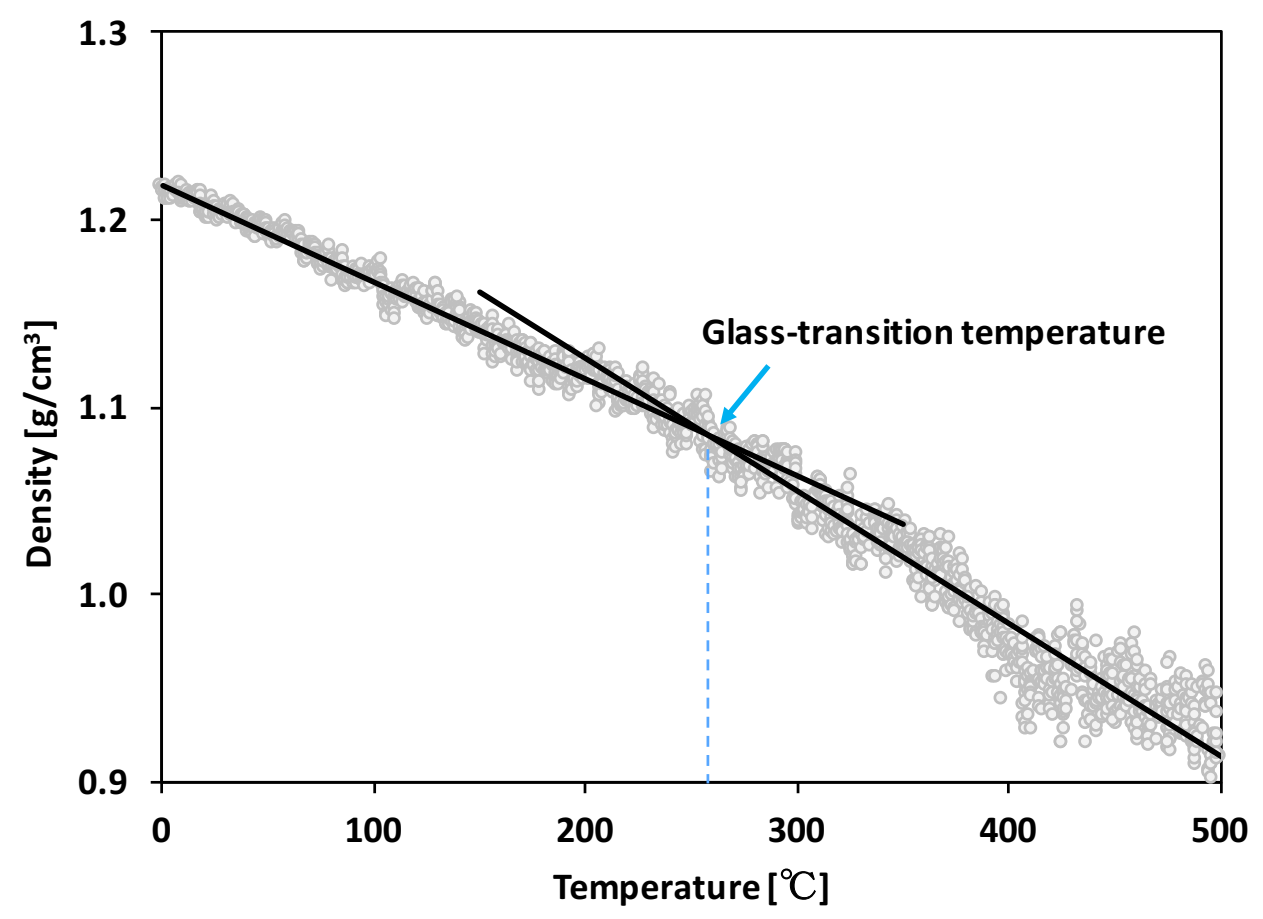

Figure 16 Plot of mass density vs temperature for determining glass-transition temperature for a EEC/ACA model of $80 \%$ crosslink density. 


\subsubsection{Coefficient of Linear Thermal Expansion}

The coefficient of linear thermal expansion (CLTE) was determined using the same output from the heating-up simulations for the glass-transition temperature. Specifically, the CLTE was calculated from

$$
\alpha=\frac{\beta}{3}=\frac{1}{3}\left(\frac{1}{V} \frac{\partial V}{\partial T}\right)
$$

where $\beta$ is the coefficient of volumetric thermal expansion (CVTE). For each simulation, the system temperatures and volumes were averaged every 2 ps. Figure 17 shows a representative plot between volume and temperature. A polynomial function of volume, $V(T)$, was fit to the data and differentiated with respect to the temperature (T) for Equation (12). In this study, the CLTE was calculated at $300 \mathrm{~K}\left(27^{\circ} \mathrm{C}\right)$ for each of the crosslinking levels.

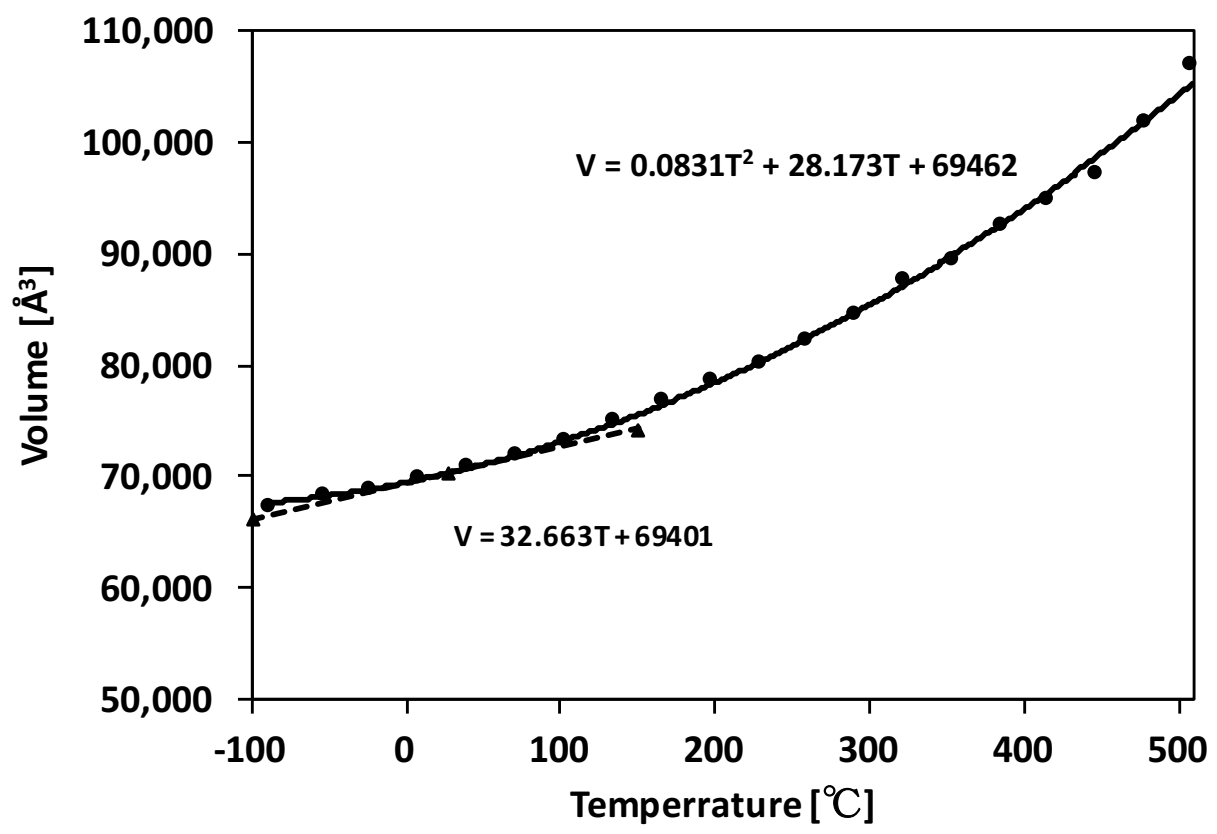

Figure 17 Plot of simulation box volume as a polynomial function of temperature from a representative $80 \%$ crosslinked cubic model with the polynomial trend line (solid line) and tangent line (dashed line) at $27^{\circ} \mathrm{C}$. 


\subsubsection{Thermal Conductivity Models}

The thermal conductivities of the crosslinked models were determined by two different methods. The first method was the Non-Equilibrium MD (NEMD) approach, also known as the direct method based on Fourier's law. The second method was the Equilibrium MD (EMD) approach based on the fluctuation-dissipation theorem, also known as the GreenKubo [70] formalism. Both methods have been previously used to predict the thermal conductivities of Si [71] and EPON862 epoxy [45].

\subsubsection{Non-equilibrium Molecular Dynamics}

The NEMD method requires a steady-state condition, and the thermal conductivity is calculated using

$$
\kappa=-\frac{J}{d T / d z}
$$

where $J$ is the heat flux (rate of heat flow per unit area) and $d T / d z$ is the temperature gradient along the z-direction. A prism-shaped MD simulation box was used to predict the thermal gradient. Figure 18 shows a representative system with an aspect ratio of 6 which this configuration was developed by Varshney et al. [72]. The prism model was divided into 20 equally-sized slabs along the length. The positions of the atoms in the slabs on both ends of the simulation box were fixed. The temperatures of the slabs next to the end slabs were held at 250 and $350 \mathrm{~K}$ (red and blue slabs in Figure 19, respectively) using the NVT ensemble with $5 \mathrm{fs}$ of temperature damping to obtain an overall temperature of $300 \mathrm{~K}$. The energies of the middle slabs were conserved with the NVE ensemble. The heat flux was calculated using

$$
J=\frac{\left(Q_{\text {hot }}-Q_{\text {cold }}\right) / 2}{A t}
$$


The total heat $\left(Q_{\text {hot }}-Q_{\text {cold }}\right)$ was divided by 2 because the heat was transferred to both sides of the simulation box. The NEMD simulation ran for $4 \mathrm{~ns}$ with $1 \mathrm{fs}$ time steps. The first $2 \mathrm{~ns}$ of the simulation established the temperature gradient into a steady state condition. The final $2 \mathrm{~ns}$ of the simulation was used to determine the average temperature in each slab as well as the inlet and outlet energies for calculating the thermal conductivity using Equation (13).

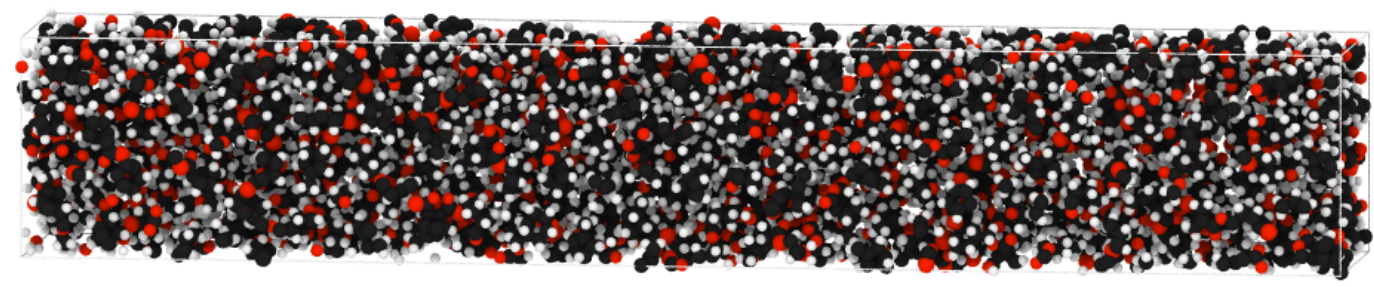

Figure 18 A representative prism-shaped model for determining the thermal conductivity using NEMD.

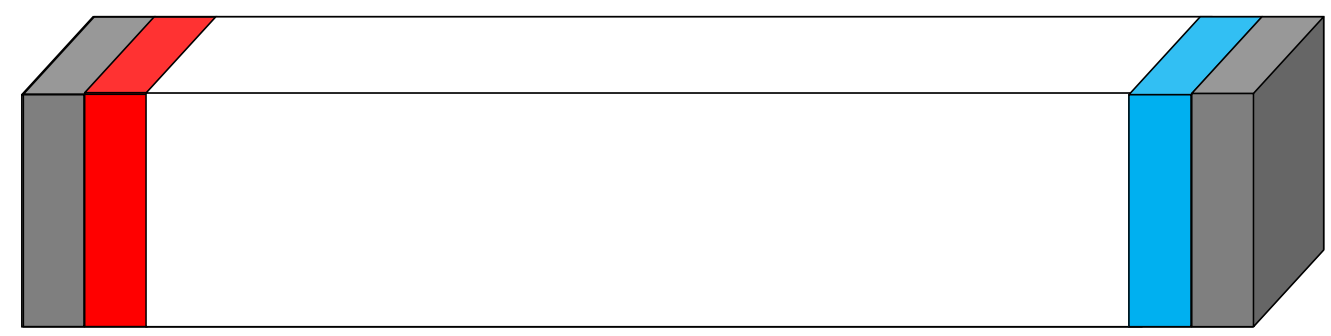

Figure 19 Simulation box including fixed slaps (grey), $350 \mathrm{~K}$ slab (red), $250 \mathrm{~K}$ slab (blue), and middle slabs (white). 


\subsubsection{Equilibrium Molecular Dynamics}

The EMD method is based on Green-Kubo formalism which is capable to calculate several transport coefficients such as viscosity, self-diffusion coefficient, thermal conductivity, etc. For the thermal conductivity, Green-Kubo [70] expression is shown in following equation.

$$
\kappa_{x}=\frac{V}{\mathrm{k}_{\mathrm{B}} \mathrm{T}^{2}} \int_{0}^{\infty}\left\langle\mathrm{J}_{\mathrm{x}}(0) \mathrm{J}_{\mathrm{x}}(\mathrm{t})\right\rangle \mathrm{dt}
$$

where $\mathrm{V}$ is the system volume, $\mathrm{k}_{\mathrm{B}}$ is the Boltzmann constant, $\mathrm{T}$ is the system temperature, and $\left\langle\mathrm{J}_{\mathrm{x}}(0) \mathrm{J}_{\mathrm{x}}(\mathrm{t})\right\rangle$ is the heat current autocorrelation function (HCACF). The EMD method can determine the thermal conductivity of an isotropic material by averaging the properties along the $\mathrm{x}, \mathrm{y}$, and $\mathrm{z}$ axes using

$$
\kappa=\frac{V}{3 k_{B} T^{2}} \int_{0}^{t_{c}}\langle J(0) \cdot J(t)\rangle d t
$$

where the heat flux vector is

$$
\mathbf{J}=\frac{1}{V}\left[\sum_{i} E_{i} \boldsymbol{v}_{i}-\sum_{i} \boldsymbol{S}_{i} \boldsymbol{v}_{i}\right]
$$

where

$$
E_{i}=\frac{1}{2} m_{i} v_{i}^{2}+\frac{1}{2} U_{i}
$$

where the first term is the total kinetic energy; $\boldsymbol{v}_{i}$ and $m_{i}$ are the velocity vector and mass

of atom $i$, respectively; and $U_{i}$ is the total potential energy of atom $i$ which includes the 12 - 6 Lennard-Jones potential for van der Waals interactions. The bonded interactions include the stretching (bond), bending (angle), and torsional (dihedral) energies. $\boldsymbol{S}_{i}$ in Equation (17) is the per-atom stress tensor of atom $i$. 
Cubic MD simulation boxes were used to determine thermal conductivities via the EMD method by initially maintaining the temperature at $300 \mathrm{~K}$ with the NVT ensemble. The simulations ran for 10 ps with 1 fs time steps with a $10 \AA$ Lennard-Jones cutoff. The "compute heat/flux" command in LAMMPS was utilized to compute the heat flux from the potential energy, kinetic energy, and stress values. The HCACF was obtained by using the autocorrelation average with the 'fix ave/correlate' command in LAMMPS.

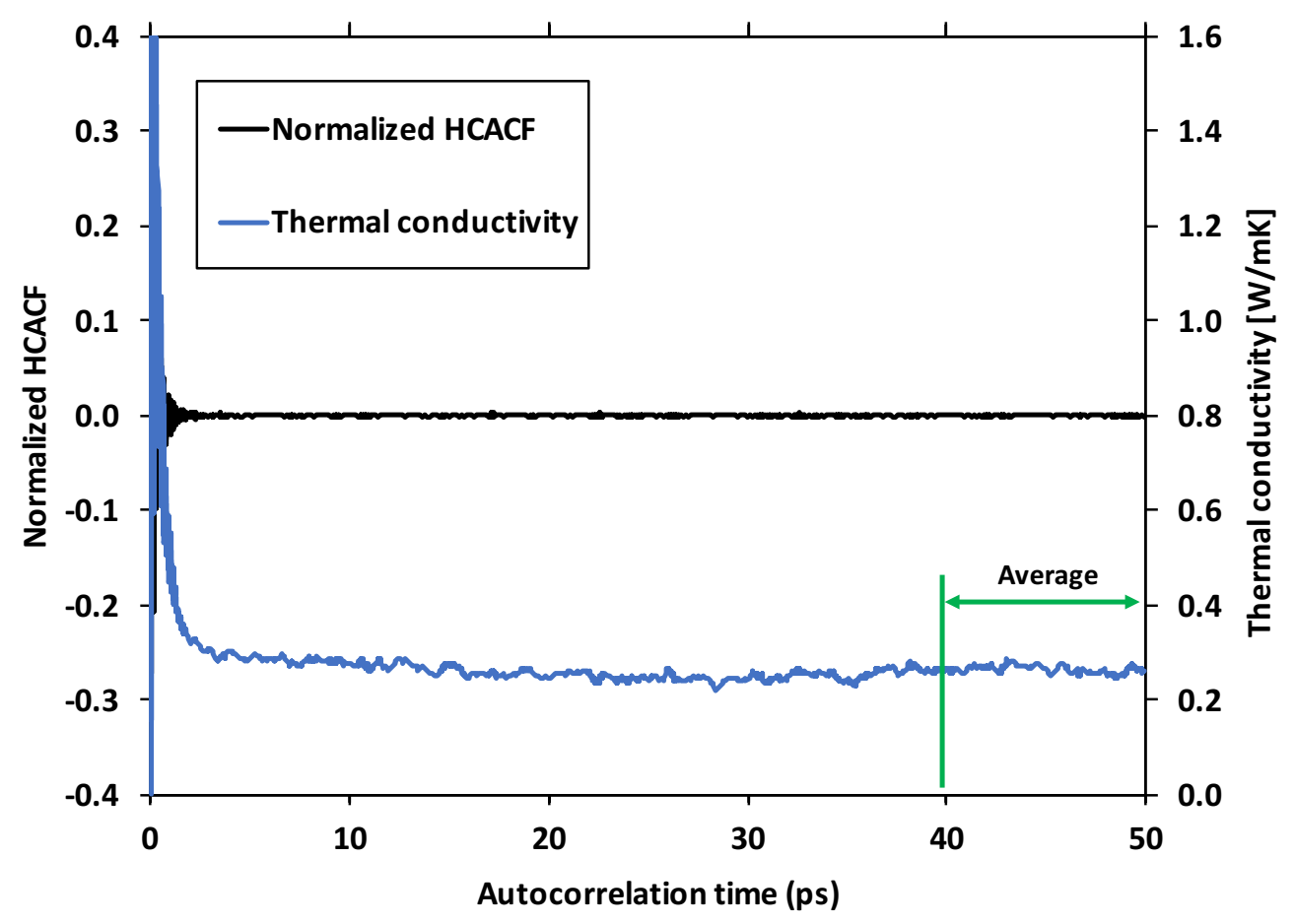

Figure 20 Representative plot of the normalized HCACF and thermal conductivity of the CE system as a function of autocorrelation time $\left(\boldsymbol{t}_{\boldsymbol{c}}\right)$.

The integral term in Equation (16) was calculated using the trapezoidal rule for $50 \mathrm{ps}$ of the correlation time $\left(t_{c}\right)$ with $5 \mathrm{fs}$ time increments. The fluctuation of the HCACF decayed to zero after $4 \mathrm{ps}$, as shown in Figure 20. The data in the figure suggests that the correlation time $\left(t_{c}=50 \mathrm{ps}\right)$ is sufficiently large for this system. The total simulation time used to determine the thermal conductivity was $10 \mathrm{~ns}$ in order to have sufficient statistical averaging. The thermal conductivity of the EEC/ACA system was calculated from equation 
(16) and is shown in Figure 20. The thermal conductivity was averaged from the final 10 ps of the correlation time.

\subsubsection{Micromechanics}

The effective elastic modulus and bulk thermal conductivity of the unidirectional carbon fiber/CE composite was predicted using micromechanics calculations. Specifically, the thermal conductivities of the epoxy matrix were taken from the MD simulations described above and the AS4 carbon fiber properties were taken from the literature $[73,74]$ shown in Table 4. The uncertainties in the elastic properties and thermal conductivities for the neat epoxy represent the standard deviation from the multiple MD predictions. Three different values of the properties of the neat epoxy were used in the micromechanics predictions: the mean value and the mean value \pm one standard deviation. This provided the uncertainty in the properties at the bulk level based on the uncertainty at the molecular level.

The Micromechanics Analysis Code based on the Generalized Method of Cells (MAC/GMC) [75] was used for the micromechanics predictions. A circular fiber approximation (rectagular pack or 'Architecture 13' as implemented in MAC/GMC) was used as a repeating unit cell (RUC). This RUC has $26 \times 26$ subcells in which 312 middle subcells represented the fiber region and the other subcells represented the matrix region. The calculation of the effective elastic modulus and thermal conductivities of the Carbon fiber/CE composite included fiber volume fractions ranging from 5\% to $80 \%$ with $5 \%$ increments.

Table 4 Materials properties of neat CE and carbon fiber.

\begin{tabular}{ccc}
\hline Properties & Neat CE (EEC/ACA) & Carbon Fiber (AS4) \\
\hline$E_{A}(\mathrm{GPa})$ & $2.38 \pm 0.35$ & 235 \\
$E_{T}(\mathrm{GPa})$ & $2.38 \pm 0.35$ & 15 \\
$v_{A}$ & $0.38 \pm 0.03$ & 0.2 \\
$v_{T}$ & $0.38 \pm 0.03$ & 0.07 \\
$G_{A}(\mathrm{GPa})$ & $0.82 \pm 0.15$ & 27 \\
$k_{A}(\mathrm{~W} / \mathrm{mK})$ & $0.24 \pm 0.02$ & 6.50 \\
$k_{T}(\mathrm{~W} / \mathrm{mK})$ & $0.24 \pm 0.02$ & 2.18 \\
\hline
\end{tabular}




\subsection{Experimental Testing}

\subsubsection{Fabrication of neat $\mathrm{CE}^{*}$}

The EEC/ACA neat epoxy [76] was fabricated using a FlackTek SpeedMixer DAC 150.1 FVZ by pouring resin and hardener in a $100 \mathrm{~g}: 138 \mathrm{~g}$ ratio into 5 mixing cups of $90 \mathrm{~g}$ each, for a total batch size of $450 \mathrm{~g}$. Each cup was mixed in the SpeedMixer for 2 minutes at $2500 \mathrm{rpm}$. The cups were degassed at $100{ }^{\circ} \mathrm{C}$ and $29 \mathrm{inHg}$ vacuum until no more bubbles appeared. The mixture was then poured into a pre-heated mold and degassed again under full vacuum. The mold was coated with Mann Ease Release 300. For the neat epoxy, the preheating and final degassing were performed at $100^{\circ} \mathrm{C}$. The curing cycle for all the epoxy specimens was $100{ }^{\circ} \mathrm{C}$ for 1 hour, then heated to $200{ }^{\circ} \mathrm{C}$ at $2^{\circ} \mathrm{C} / \mathrm{min}$, then held at $200{ }^{\circ} \mathrm{C}$ for two hours. The oven was turned off and the cured epoxy was allowed to cool in the oven to room temperature at a $\sim 1{ }^{\circ} \mathrm{C} / \mathrm{min}$ cooling rate. The molds made about 20 rectangular bars (165 mm long by $19 \mathrm{~mm}$ wide by $3.3 \mathrm{~mm}$ thick) and 5 disks $(64 \mathrm{~mm}$ diameter and $3.2 \mathrm{~mm}$ thick), from which the testing specimens were machined. The density of the samples was measured at $23{ }^{\circ} \mathrm{C}$ according to ASTM D792 [77].

\subsubsection{Tensile Testing*}

The tensile properties (at $23{ }^{\circ} \mathrm{C}$, ASTM type I sample geometry) were measured according to ASTM D638 [78] at a crosshead rate of $1 \mathrm{~mm} / \mathrm{min}$. An InstruMet Sintech screw-driven mechanical testing machine was used with an axial extensometer to collect strain values. The tensile strength, strain, and modulus [76] were measured at $23{ }^{\circ} \mathrm{C}$.

\subsubsection{DMA testing for glass-transition temperature*}

Dynamic Mechanical Analysis (DMA) was used to find the glass-transition temperature of the epoxy samples. The tests were conducted with a TA Instruments Q800 DMA using the dual/single cantilever clamp. The single cantilever geometry was used for all testing, with

\footnotetext{
* This section was performed and wrote by Julie M Tomasi.
} 
a set length of $17.5 \mathrm{~mm}$ between clamps. The sample dimensions were $3.3 \mathrm{~mm}$ thick, 12 $\mathrm{mm}$ wide, and $35 \mathrm{~mm}$ long; and were cut from the rectangular bars. Samples were rinsed with isopropyl alcohol before testing. Each sample was loaded into the clamps and tightened down using 8 in-lb of torque. Tweezers were used to keep the sample surfaces free of oil from hands and fingers.

Once the samples were in placed in the clamps, amplitude sweep tests were performed. The test mode was set to "DMA Multi Strain" with the test set to "Strain Sweep". The frequency was kept at $1 \mathrm{~Hz}$ with the isothermal temperature at $35^{\circ} \mathrm{C}$ and a soak time of 5 minutes. The amplitude was swept from $5 \mu \mathrm{m}$ to $50 \mu \mathrm{m}$. Following these tests, graphs of amplitude versus loss modulus were analyzed to determine the amplitude needed for the temperature sweep test.

To determine the storage modulus, loss modulus, and tan delta, the program mode was set to "DMA multi frequency strain" and the test protocol was set to "temp ramp/frequency sweep". The procedure parameters included an amplitude of $30 \mu \mathrm{m}$, an initial temperature of $50{ }^{\circ} \mathrm{C}$ with a soak time of 5 minutes, a temperature ramp rate of $3{ }^{\circ} \mathrm{C} / \mathrm{min}$, and a final temperature of $290{ }^{\circ} \mathrm{C}$ with a hold time of 5 minutes. The frequency was held at $1 \mathrm{~Hz}$ for the entire duration of the tests. Once the tests were completed, the data was analyzed to determine the storage modulus, loss modulus, and tan delta values for the entire temperature range. The temperature at the peak of the tan delta graph provided the glass transition temperature.

\subsubsection{Thermal Conductivity Testing*}

Through-plane thermal conductivity was measured using a Holometrix Model TCA-300 Thermal Conductivity Analyzer, which follows the ASTM F433 [79] guarded heat flow meter method. The though-plane thermal conductivities of the $3.2 \mathrm{~mm}$ thick, $50 \mathrm{~mm}$ diameter disc-shaped test specimens were measured at $55^{\circ} \mathrm{C}$.

\footnotetext{
* This section was performed and wrote by Julie M Tomasi.
} 


\subsection{Results}

\subsubsection{Mass Density}

The average mass densities of the cubic and prism models of cured EEC/ACA system at each crosslink density are shown in Table 3 . The mass density values are in good agreement with the experimentally obtained value of $1.219 \pm 0.001 \mathrm{~g} / \mathrm{cm}^{3}$. In general, the predicted mass densities of both cubic and prism MD models decreased with increases in crosslink density. The mass density reduction is likely a result of the decrease in chain flexibility with increased in crosslink density. At lower crosslink densities, the monomers can be positioned closer together with little constraint. At higher crosslink densities, the additional covalent bonds between monomers increase constraining forces, thus prohibiting full structural densification.

\subsubsection{Mechanical Properties}

For mechanical properties of EEC/ACA system, five crosslinked models are different EEC/ACA models from the models for thermal properties. These models were crosslinked by only 100 cycles of crosslink shown in Schematic 1 . The average crosslink density and mass density of EEC/ACA system for mechanical properties are shown in Table 5. The mass density is good agreement with the experimental value $\left(1.219 \pm 0.001 \mathrm{~g} / \mathrm{cm}^{3}\right)$.

Table 5 The average elastic properties of EEC/ACA from MD.

\begin{tabular}{ll}
\hline Properties & Value \\
\hline Crosslink density & $0.74 \pm 0.06$ \\
Mass density $\left(\mathrm{g} / \mathrm{cm}^{3}\right)$ & $1.208 \pm 0.010$ \\
$\mathrm{E}(\mathrm{MPa})$ & $2328 \pm 351$ \\
$\mathrm{G}(\mathrm{MPa})$ & $816 \pm 152$ \\
$v$ & $0.38 \pm 0.03$ \\
\hline
\end{tabular}


The predicted elastic properties are shown in Table 5, which are averaged over five replicate models in all three Cartesian directions. Figure 21 shows the Young's modulus as a function of crosslink density, as well as the experimental value which the crosslink density is unknown. The Young's modulus of the 0.79 and 0.80 of crosslink-density models, which is about $2700 \mathrm{MPa}$, agrees well with the Young's modulus from experiment.

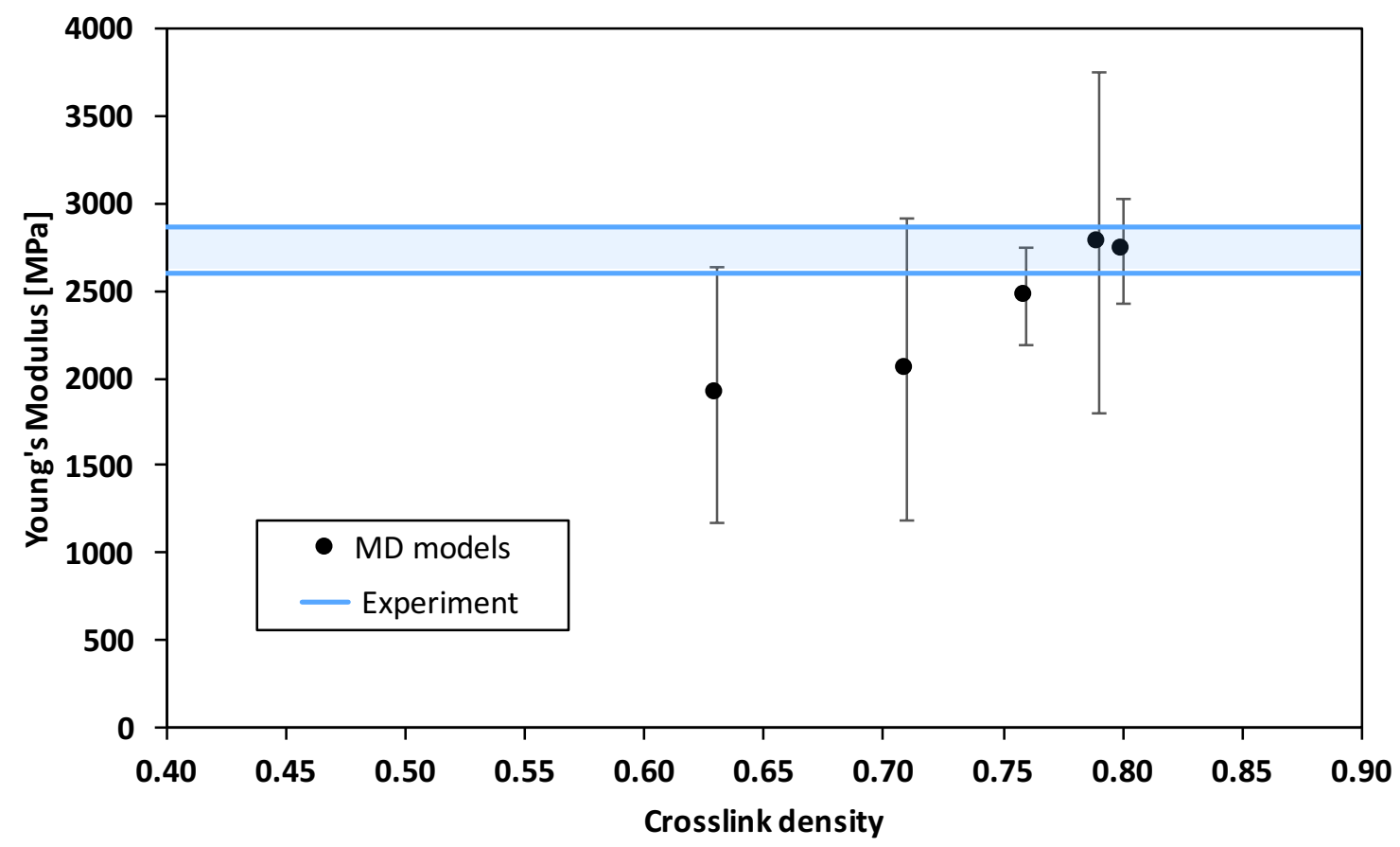

Figure 21 Plot of Young's modulus as a function of crosslink density compared with experiment [76]. 
Figure 22 shows the predicted axial and transverse elastic modulus of carbon fiber/EEC/ACA composites. The standard deviations of axial elastic modulus are relatively small which the error bars are hidden in the data points. For the transverse direction, the standard deviations are shown in the plot. These predicted axial and transverse elastic modulus are in good agreement with the experimental values form Kilmek-McDonald [80] which validate the multiscale computational method modeling for carbon fiber/CE system.

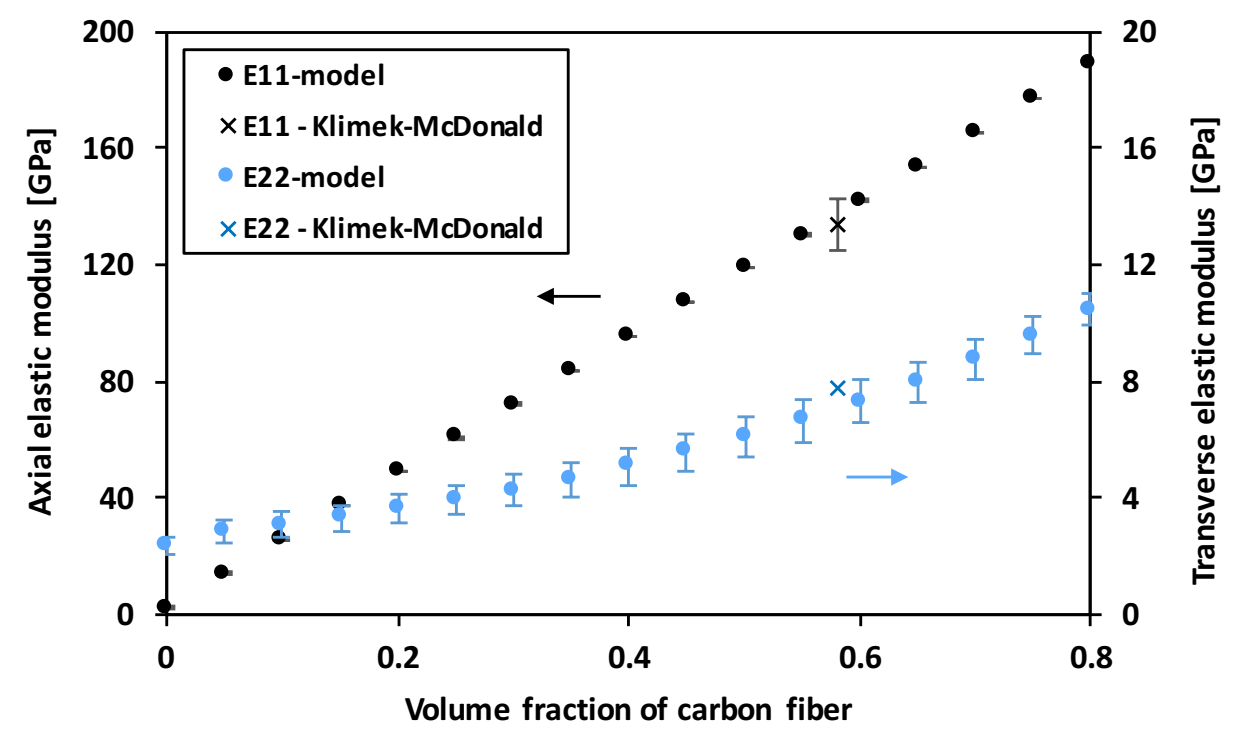

Figure 22 Predicted axial and transverse elastic modulus as the function of carbon fiber volume fraction. Experimental values of carbon fiber/EPON862 composites are obtained from KilmekMcDonald [80]. 


\subsubsection{Thermal Properties}

\subsubsection{Glass-Transition Temperature}

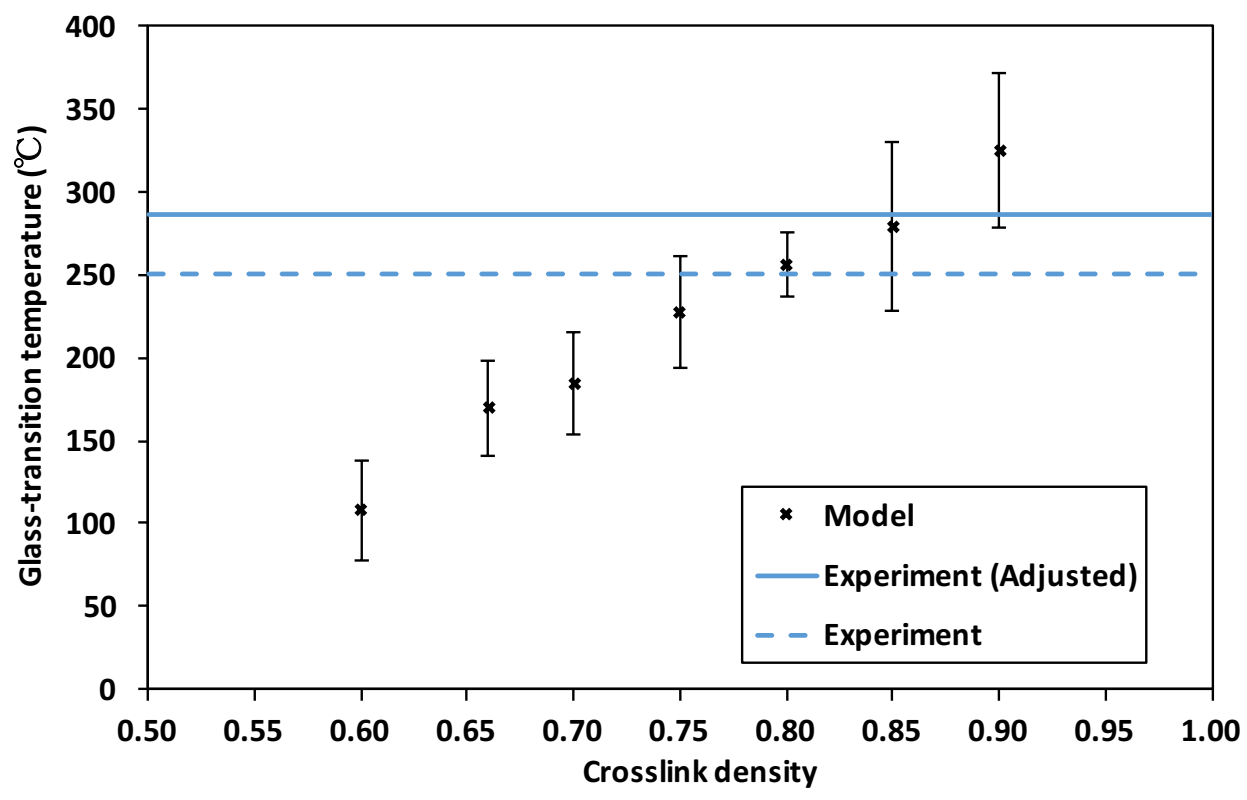

Figure 23 Predicted glass-transition temperatures of cured epoxy models as a function of crosslink density. Experimentally obtained value (with unknown crosslink density) is overlaid on the data.

Figure 23 shows the glass-transition temperature averaged over five different models for each crosslink density. The plot shows that the glass-transition temperature is crosslink density dependent, as was previously observed with the EPON862 system [46]. The experimental glass-transition temperature (dashed line) was $250.58 \pm 1.05{ }^{\circ} \mathrm{C}$. The experimental heating rate is about 12 order of magnitude slower than the heating rate in MD simulation. Due to the heating rate effect, the glass-transition temperature increases 3 ${ }^{\circ} \mathrm{C}$ when the heating rate increases an order of magnitude [47, 81]. The adjusted glasstransition temperature (solid line) is corrected by adding $36{ }^{\circ} \mathrm{C}$ to the experimental (original) value. The predicted glass-transition temperature for 85 and $90 \%$ crosslink densities are in good agreement with the experiment, for which the crosslink density is not known. 


\subsubsection{Coefficient of Linear Thermal Expansion}

Figure 24 shows the predicted CLTE of the epoxy as a function of crosslink density at 300K. A range of experimental CLTE values from the literature [82-86] (with unknown crosslink density) are included in the plot. The plot demonstrates that the predicted CLTE decreases with increasing crosslink density. A similar trend for the CLTE of a CE system was observed by Komarov et al. [59]. From the figure it is also evident that the predicted CLTE values approach the experimental values at increasing levels of crosslink density. Because most epoxies are expected to have relatively high crosslink densities, there is satisfactory agreement between models and literature values.

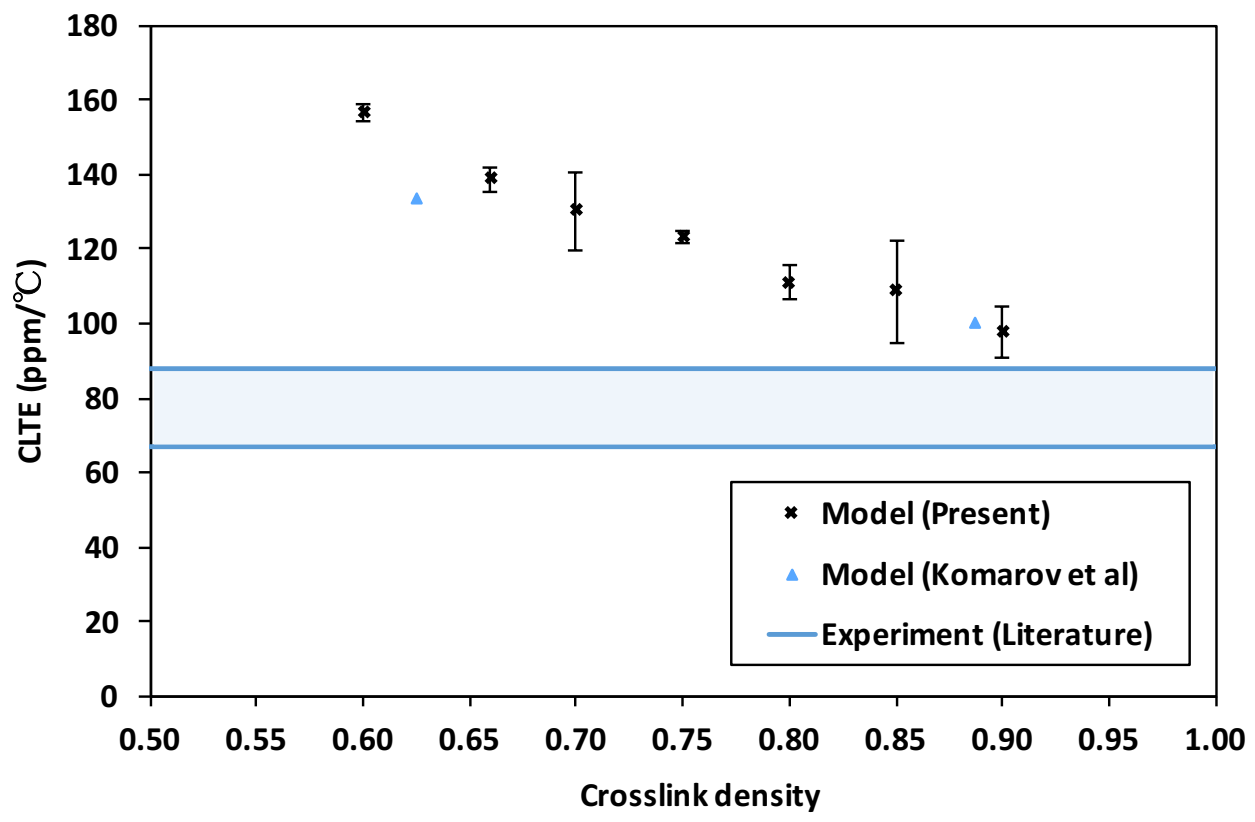

Figure 24 Plot of predicted CLTE as a function of crosslink density at $300 \mathrm{~K}$. Literature (experiment) values of unknown crosslink density are overlaid and predicted values from literature are also included. 


\subsubsection{Thermal Conductivity}

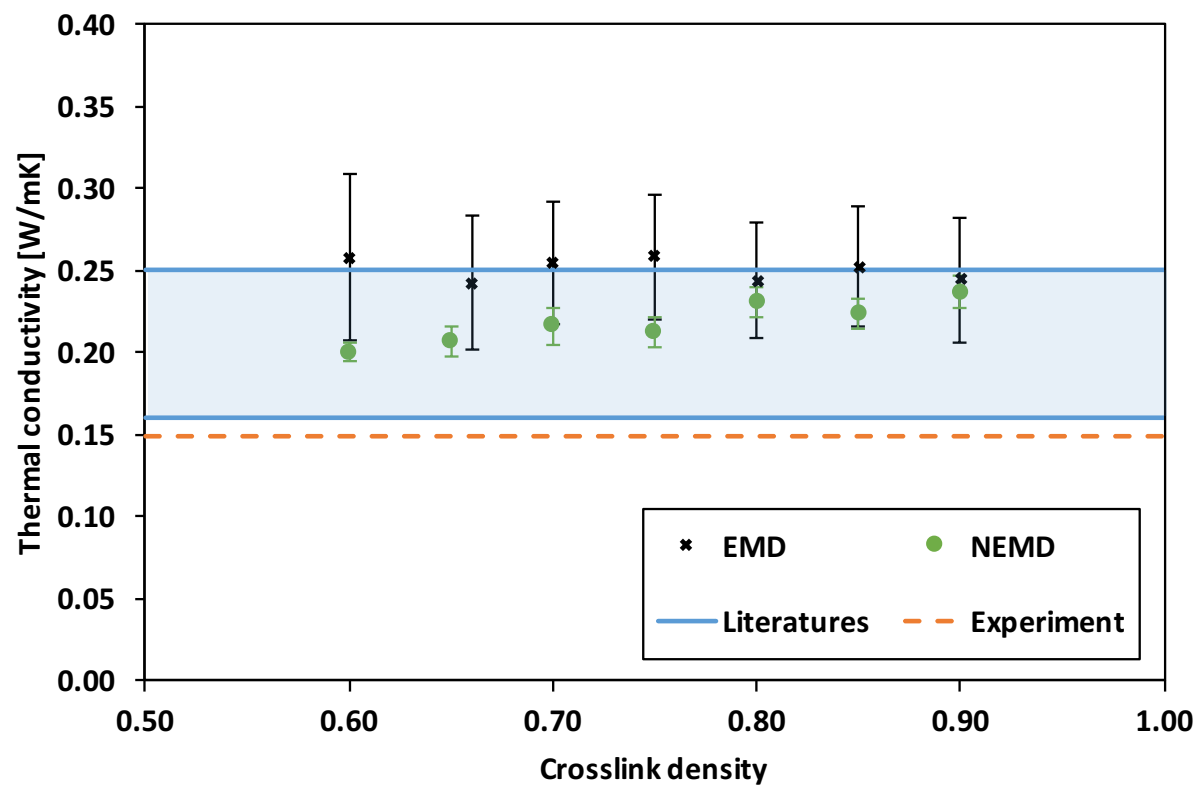

Figure 25 Plot of NEMD and EMD thermal conductivities as a function of crosslink density compared with experiments (Blue region [87-93] and orange line [76]).

Figure 25 provides the predicted average thermal conductivities of the CE epoxy system averaged over the five models using the EMD and NEMD methods. The error bars represent the standard deviations associated with the five models at each crosslink density. Given the uncertainty levels, the results suggest that the two methods are in general agreement. The thermal conductivity from NEMD is dependent on the crosslink density but the EMD cannot capture the crosslinking dependence. This behavior is similar to that observed with MD modeling of the EPON862 system [45]. The results also suggest that the predicted thermal conductivities are within the range of the experimental thermal conductivity values reported in the literature [87-93]. However, the predicted thermal conductivities are significantly higher than those from the experiment [76] described herein. The source of the discrepancy is uncertain, however, it is clear that the agreement with the literature values serves as a validation of the simulation techniques. 


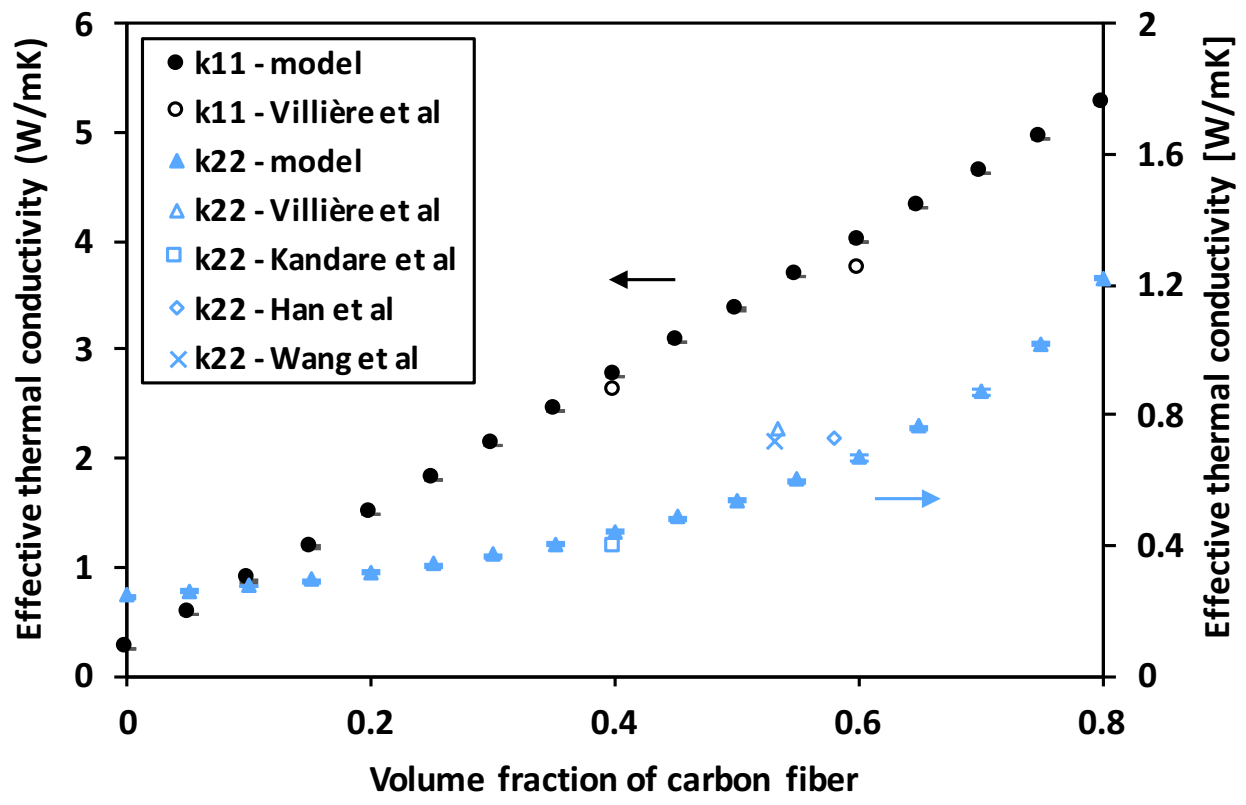

Figure 26 Plot of thermal conductivity as a function of carbon fiber volume fraction parallel (circle) and transverse (triangle) to the fiber direction. Experimental values taken from Villière et al. [94-97].

Figure 26 shows the predicted thermal conductivities of a unidirectional EEC/ACA/carbon fiber composite parallel $\left(\mathrm{k}_{11}\right)$ and transverse $\left(\mathrm{k}_{22}=\mathrm{k}_{33}\right)$ to the fiber direction. The effective axial and transverse thermal conductivities increase linearly and nonlinearly, respectively, with the carbon fiber volume fraction. The standard deviations of these calculations are relatively small and the corresponding error bars are partially hidden by the data points in Figure 26. The predicted thermal conductivities are in good agreement with experimental values from the literature [94-97], thus further validating the multiscale computational modeling method. 


\subsection{Conclusions}

There are two important results from this chapter. First, an all-atom MD modeling method for $\mathrm{CE}$ epoxies was developed and validated with comparison to experimental data for glass transition temperature, CLTE, and thermal conductivity. To the best of the authors' knowledge, this is the first such method developed specifically for CE epoxies. Second, the multiscale modeling approach developed in this study, which uses results of MD and micromechanical modeling, can accurately predict the bulk-level thermal conductivity of CE-based composite materials. The multiscale approach was validated with comparison of predicted thermal conductivity values of epoxy/carbon fiber composites to those found in the literature.

The developed multiscale modeling method can be used to facilitate the development of CE-based epoxy composites and nanocomposite materials for thermal applications. By taking advantage of low-cost simulations to establish preliminary material designs, overall materials development costs can be dramatically reduced and development times can be expedited. This ability to efficiently develop new CE-based composite materials is particularly important for the continued development of ACCC high-transmission power lines. 


\section{CHAPTER 4 MULTISCALE MODELING: THERMAL CONDUCTIVITY OF PRISTINE GRAPHENE NANOPLATELET/CARBON FIBER/CYCLOALIPHATIC EPOXY HYBRID COMPOSITES}

\subsection{Introduction}

Because cycloaliphatic epoxies (CE) have excellent chemical resistance and a high glasstransition temperature relative to other polymers used in composite materials, they are commonly used for applications with aggressive environments. Carbon fiber $(\mathrm{CF}) / \mathrm{CE}$ composites have been used as structural reinforcement in aluminum conductor composite core (ACCC) [98] high-voltage power lines resulting in a six-fold reduction of high temperature sag when compared with the conventional aluminum conductor steelreinforced (ACSR) cable. ACCC lines must be able to endure extreme weather conditions and localized heating due to transmission line faults. However, $\mathrm{CE}$ has very low thermal conductivity. To improve the material resistance to localized heating (or hotspots), we seek to increase the thermal conductivity of the $\mathrm{CE}$ matrix by incorporating conductive nanofillers.

Graphene Nanoplatelet (GNP) reinforcement has been shown to improve the mechanical properties in the transverse direction of GNP/CF/Epoxy hybrid composite layers [33, 36] and increase the thermal conductivity $[11,14,15,22,24,27-31,40]$. This is because of the superior mechanical and thermal properties of pristine graphene. The Young's modulus of graphene is equal to $1 \mathrm{TPa}$ [7], and the in-plane thermal conductivity is about 1,000-5,300 $\mathrm{W} / \mathrm{mK}[5,6,99]$. GNP has capability to enhance the thermal conductivity of the CE system.

However, it is still unclear the degree to which GNP dispersion affects the composite thermal conductivity of CE-based composites. An accurate model can establish what conductivity values are expected given perfect GNP dispersion. Knowing the ideal case 
would enable experimentalists to better assess the dispersion of their samples and determine whether improved dispersion is desirable or if the benefits are likely minimal. Recently, a method was developed by Chinkanjanarot et al. [100] to predict the thermal conductivity of neat $\mathrm{CE}$ resins using molecular dynamics (MD) simulation, specifically, a non-equilibrium MD (NEMD) approach. The NEMD can predict the thermal conductivity of neat CE model good agreement with experiment. Although this method works nicely for isotropic polymer resins, it can only determine the thermal conductivity in one particular direction for a single simulation and typically requires an elongated simulation box. Applying NEMD to anisotropic materials necessitates several separate simulations to determine the directional-dependent conductivity, and multiple elongated boxes having the same GNP mass fraction may be needed. Thus, it is difficult to use NEMD in modeling composite materials. Alternatively, Equilibrium Molecular Dynamics (EMD) can be used to determine the three-dimensional thermal conductivity in a single simulation and requires a standard cubic simulation box. The EMD method has been used to successfully study the thermal conductivity of epoxy systems $[45,57]$.

In this study, the objective is to predict the bulk thermal conductivity of fully cured GNP/CE nanocomposites and GNP/CF/CE hybrid composites using a multiscale modeling approach (including MD, randomization, and micromechanics). The influence of GNP dispersion and GNP concentration on the effective thermal conductivity is analyzed. The predicted thermal conductivities compare well with the literature experimental values. The results show that the dispersion of GNP directly influences the transverse thermal conductivity of the hybrid composites.

\subsection{Computational Details}

The multiscale modeling scheme is shown in Figure 27. The thermal conductivity of GNP/CE nanocomposites was initially determined at the molecular level by MD. The effective nanocomposite thermal conductivity was obtained by randomization and then 
scaled with different amounts of GNP by micromechanics. The effective thermal conductivity of $\mathrm{GNP} / \mathrm{CF} / \mathrm{CE}$ hybrid composites was also determined by using micromechanics.

\subsubsection{Molecular Modeling}

All Molecular Minimization (MM) and MD simulations were performed on Large-scale Atomic/Molecular Massively Parallel Simulator (LAMMPS) [62] with Optimized Potential for Liquid Simulation All Atom (OPLS-AA) [63] force field. All molecular graphics were rendered by using Open Visualization Tool (OVITO) [65].

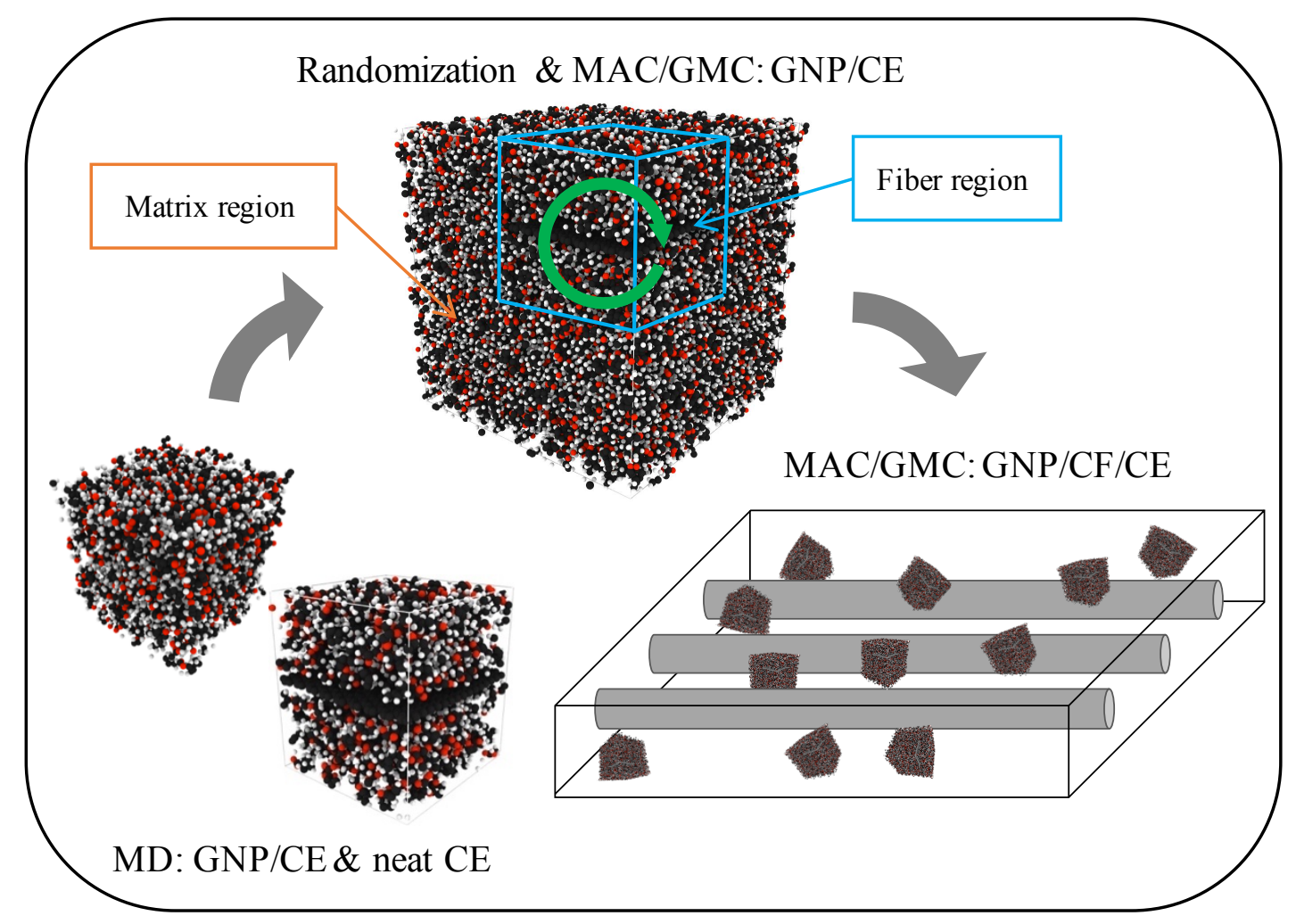

Figure 27. Schematic of multiscale modeling. Red: oxygen, black: carbon, white: hydrogen.

The molecular structures of epoxy cyclohexyl methyl 3,4 epoxy cyclohexyl carboxylate (EEC), which is a CE resin, and the anhydride curing agent (ACA) were created by using a similar procedure described in previous studies $[26,46,50,100]$. The molecular structure 
of Modified ACA [100], which is necessary to initiate curing, was also created using the same method. The molecular structures of these monomers are shown in Figure 28.

\subsubsection{GRAPHENE MD MODEL}

Graphene was created by using the lattice command in LAMMPS. The lattice vectors were $a_{1}(1,0,0), a_{3}(0,1.732,0), a_{3}(0,0,3.350)$ with $2.46 \AA$ lattice length [101]. The basis of each carbon atom is given in Table 7. Graphene models of 1,2,3, and 4 layer(s) of graphene were built with 576 atoms per layer. The molecular structure of double-layer GNP is shown in Figure 28. Bonds, angles, and dihedrals were determined through the periodic boundary. All graphene models were equilibrated with NVT at $300 \mathrm{~K}$. A cutoff radius of $10 \AA$ was selected for pairwise interactions. The equilibration was run for $1 \mathrm{~ns}$ with 1 fs time steps.

\subsubsection{NEAT CE AND GNP/CE MODELS}

The CE system was combined with graphene as shown in Figure 28. In the CE system, the molecular ratio of the EEC, ACA, and modified ACA molecules was $7: 13: 1$ representing the stoichiometry between $\mathrm{CE}$ and anhydride molecule which is $1: 2$, respectively. Since all models in this research were set to be cubic, the ratio of atoms between GNP and CE depended on the number layers of GNP as listed in Table 7. Prior to forming the cubic model, the CE and GNP were placed into a long box. For the neat CE model, all EEC, ACA, and modified ACA were combined without GNP. 
Table 6 The factional coordinates of carbon atom in graphene unit cell.

\begin{tabular}{cc}
\hline No. of basis atom & Fractional coordinates \\
\hline 1 & $(0,0,0)$ \\
2 & $(0.5,1.667,0)$ \\
3 & $(0.5,0.5,0)$ \\
4 & $(0,0.667,0)$ \\
5 & $(0,0.333,0.5)$ \\
6 & $(0.5,0.5,0.5)$ \\
7 & $(0.5,0.833,0.5)$ \\
8 & $(0,0,0.5)$ \\
\hline
\end{tabular}

Table 7 The numbers of total atoms and GNP mass fraction of each system.

\begin{tabular}{ccccc}
\hline System & GNP atoms & CE atoms & Total atoms & GNP mass fraction \\
\hline Neat CE & 0 & 7,164 & 7,164 & 0.000 \\
$1 \mathrm{GNP} / \mathrm{CE}$ & 576 & 5,373 & 5,949 & 0.151 \\
2GNP/CE & 1,152 & 4,776 & 5,928 & 0.287 \\
3GNP/CE & 1,728 & 4,179 & 5,907 & 0.408 \\
4GNP/CE & 2,304 & 3,582 & 5,886 & 0.517 \\
\hline
\end{tabular}

The long simulation box was alternately compressed between energy minimization and box compressing by "fix deform" command along with NVT ensemble. The final lengths of pre-crosslinked simulation box for each system are shown in Table 8. The densification performed with four different compressing steps shown in Table 9 which ran for $5.2 \mathrm{~ns}$ with 1 fs time steps. The stepwise technique prevented the residual stress of the model during the densification. Five different model of uncrosslinked GNP/CE were obtained for each system.

Table 8 The length of the simulation boxes after the densification.

\begin{tabular}{cccc}
\hline \multirow{2}{*}{ System } & \multicolumn{3}{c}{ Final length of densified box $(\AA)$} \\
\cline { 2 - 4 } & $\mathrm{x}_{\mathrm{f}}$ & $\mathrm{y}_{\mathrm{f}}$ & $\mathrm{z}_{\mathrm{f}}$ \\
\hline 1GNP/CE & 38.69 & 37.70 & 36.18 \\
2GNP/CE & 38.69 & 37.70 & 36.05 \\
3GNP/CE & 38.69 & 37.70 & 35.92 \\
4GNP/CE & 38.69 & 37.70 & 35.79 \\
\hline
\end{tabular}


Table 9 The length ratio of the cubic and prism-shape simulation boxes during the densification.

\begin{tabular}{ccccc}
\hline \multirow{2}{*}{ Step } & \multicolumn{3}{c}{ Length ratio } & Time (ns) \\
\cline { 2 - 4 } & $\mathrm{x}$ & $\mathrm{y}$ & $\mathrm{z}$ & 1.2 \\
\hline 1 & $4 \mathrm{x}_{\mathrm{f}}$ & $4 \mathrm{y}_{\mathrm{f}}$ & $4 \mathrm{z}_{\mathrm{f}}$ & 2 \\
2 & $1.15 \mathrm{x}_{\mathrm{f}}$ & $1.15 \mathrm{y}_{\mathrm{f}}$ & $1.15 \mathrm{z}_{\mathrm{f}}$ & 1 \\
3 & $1.05 \mathrm{x}_{\mathrm{f}}$ & $1.05 \mathrm{y}_{\mathrm{f}}$ & $1.05 \mathrm{z}_{\mathrm{f}}$ & 1 \\
4 & $\mathrm{x}_{\mathrm{f}}$ & $\mathrm{y}_{\mathrm{f}}$ & $\mathrm{z}_{\mathrm{f}}$ & 1 \\
\hline
\end{tabular}
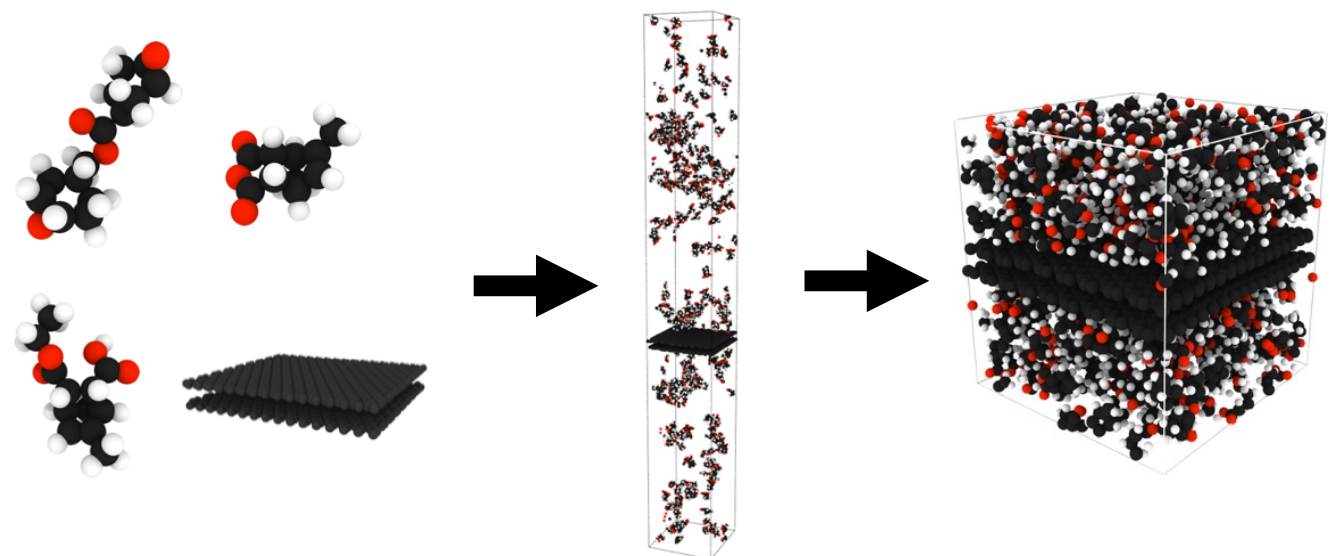

Figure 28. Combining of EEC, ACA, modified ACA and GNP into a long simulation box. Then, compressing to the densified model. Black, red, and white color spheres represent carbon, oxygen, hydrogen atoms, respectively.

The crosslinking process consisted of two main reactions as described in our previous work [100] but was executed until the crosslink conversion reached 80 percent or above. During the crosslinking process, every one hundred cycles, the crosslinked model was equilibrated with the NPT ensemble using isotropic pressure of 1 ATM. This equilibration step was carried out for $1 \mathrm{~ns}$ with $1 \mathrm{fs}$ time steps. After the crosslinking process, the model was fully equilibrated for $2 \mathrm{~ns}$ with the NPT ensemble using anisotropic pressure of 1 atmosphere. As before, $1 \mathrm{fs}$ time steps were used. The mass density was calculated every 1,000 time steps and the last ten mass densities were averaged to obtain the densities listed in Table 10. The crosslinked and equilibrated models shown in Figure 29 were used to determine the thermal conductivities as described below. Five different models of each system were 
modeled for good statistical results. All crosslink percentages and mass densities are show in Table 10.

Table 10 Crosslink percentage, mass density, and thermal conductivity of each system.

\begin{tabular}{ccccc}
\hline System & $\begin{array}{c}\text { Crosslink } \\
\text { percentage }(\%)\end{array}$ & $\begin{array}{c}\text { Mass density } \\
\left(\mathrm{g} / \mathrm{cm}^{3}\right)\end{array}$ & \multicolumn{2}{c}{ Thermal conductivity $(\mathrm{W} / \mathrm{mK})$} \\
\cline { 4 - 5 } Neat CE & $80.0 \pm 0.0$ & $1.199 \pm 0.006$ & In-plane $\left(\kappa_{x}^{M D}, \kappa_{y}^{M D}\right)$ & Out-of-plane $\left(\kappa_{z}^{M D}\right)$ \\
1GNP/CE & $79.8 \pm 0.4$ & $1.243 \pm 0.025$ & $98.0 \pm 11.6$ & $0.237 \pm 0.016$ \\
2GNP/CE & $80.0 \pm 0.0$ & $1.330 \pm 0.008$ & $215.4 \pm 12.5$ & $0.192 \pm 0.026$ \\
3GNP/CE & $80.2 \pm 0.4$ & $1.401 \pm 0.007$ & $317.3 \pm 17.3$ & $0.171 \pm 0.027$ \\
4GNP/CE & $80.2 \pm 0.4$ & $1.498 \pm 0.029$ & $464.4 \pm 37.8$ & $0.151 \pm 0.026$ \\
\hline
\end{tabular}
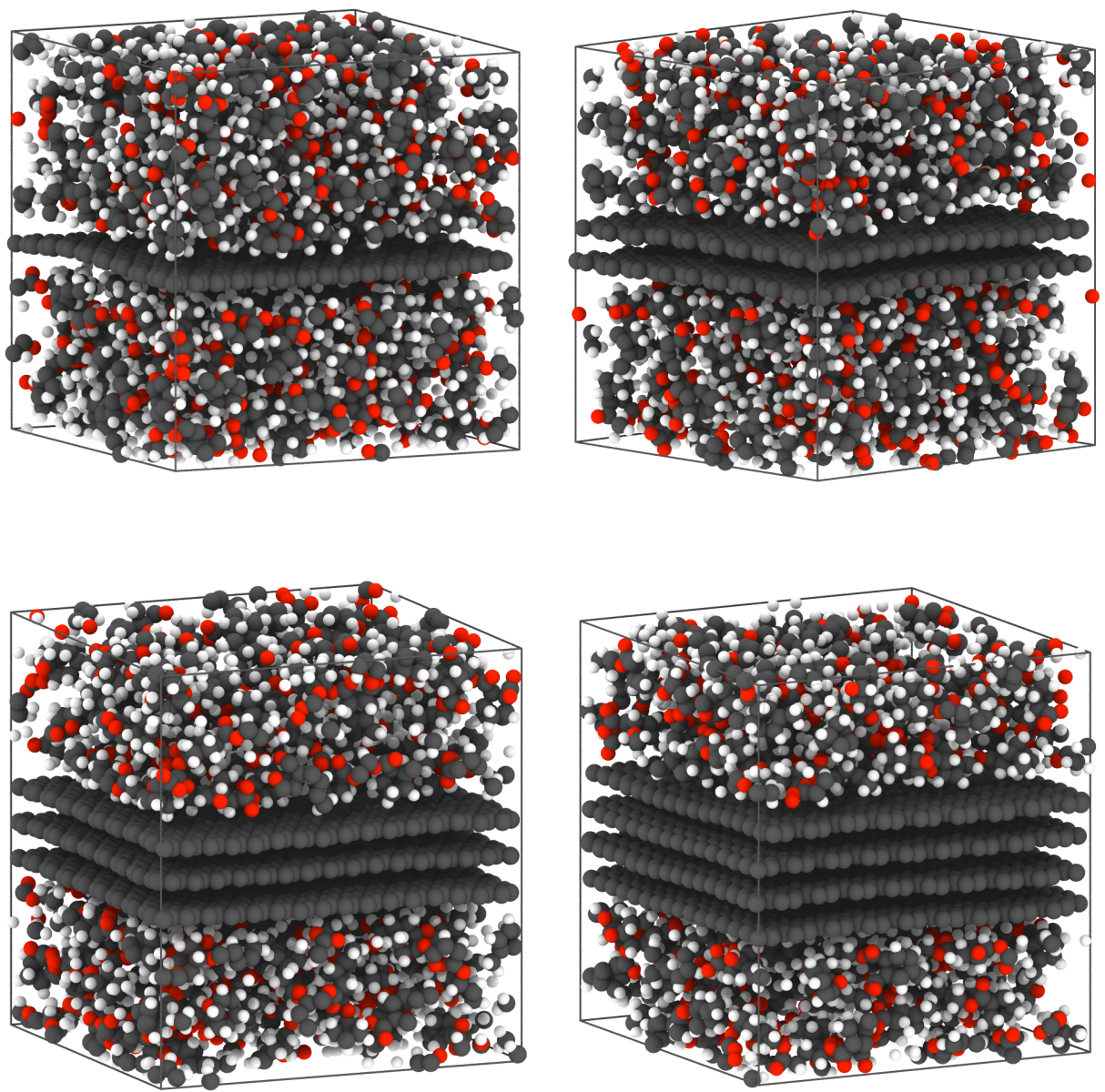

Figure 29 Representative crosslinked models of 1, 2, 3, and 4-layer GNP/CE after equilibration. 


\subsubsection{Thermal conductivity by EMD}

All thermal conductivities were determined from five independent models of each system by using the EMD method. The thermal conductivity was calculated by the Green-Kubo approach

$$
\kappa_{x}=\frac{1}{k_{B} T^{2} V} \int_{0}^{\infty}\left\langle J_{x}(0) J_{x}(t)\right\rangle d t
$$

where $k_{B}$ is Boltzmann constant, $T$ and $V$ is temperature and volume of the system, respectively, \langle\rangle denotes time average and $J_{x}(t)$ is heat flux in $\mathrm{x}$ direction at time $t$. The heat flux was calculated using the "compute heat/flux" command. Then, $\left\langle J_{x}(0) J_{x}(t)\right\rangle$, the heat flux autocorrelation function (HFACF) was obtained using the "fix ave/correlate" command. The HFACF was sampled every 5 time steps with $1 \mathrm{fs}$ time steps for a total of 10,000 samples to let the HFACF decayed to zero. From Figure 30 to Figure 33 show the HFACF of 1, 2, 3, and 4-layer GNP/CE system, respectively.

Then, the HFAFC was approximately integrated using the trapezoidal rule. The thermal conductivity ( $\kappa$ ) was determined every $50,000 \mathrm{fs}$, and the simulation ran for $10 \mathrm{~ns}$. The NVT ensemble was applied to maintain a temperature of $300 \mathrm{~K}$, and the cut-off radius of

pairwise interactions was specified at $10 \AA$. The last ten thermal conductivities for each direction were averaged to be used in the micromechanics calculations described in the follow section. 


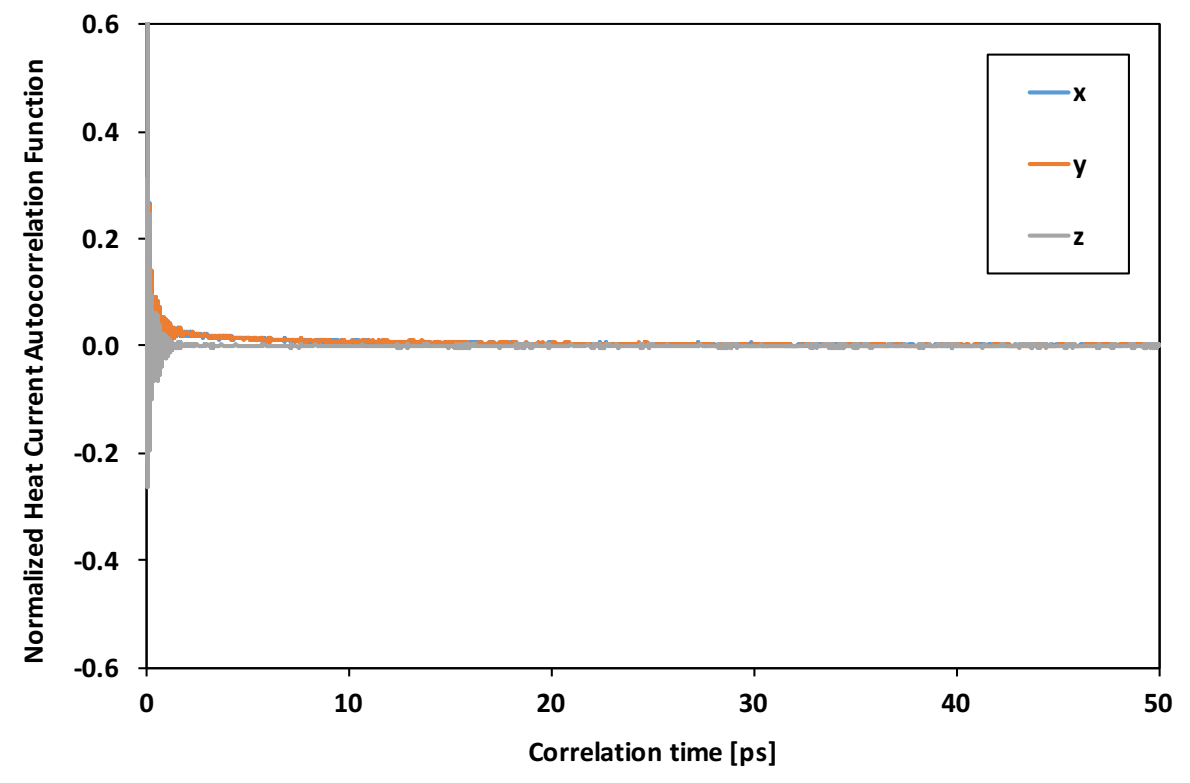

Figure 30 Normalized heat flux autocorrelation function for 1-layer GNP/CE system.

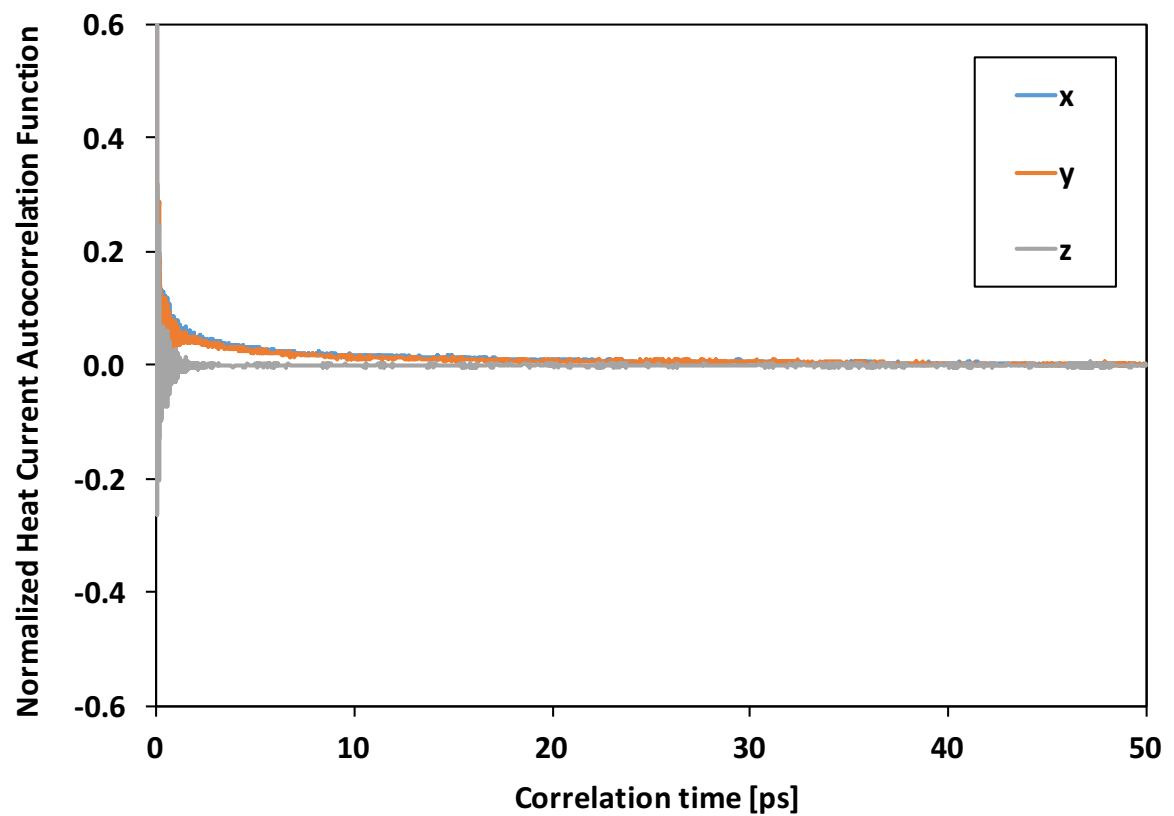

Figure 31. Normalized heat flux autocorrelation function for 2-layer GNP/CE system. 


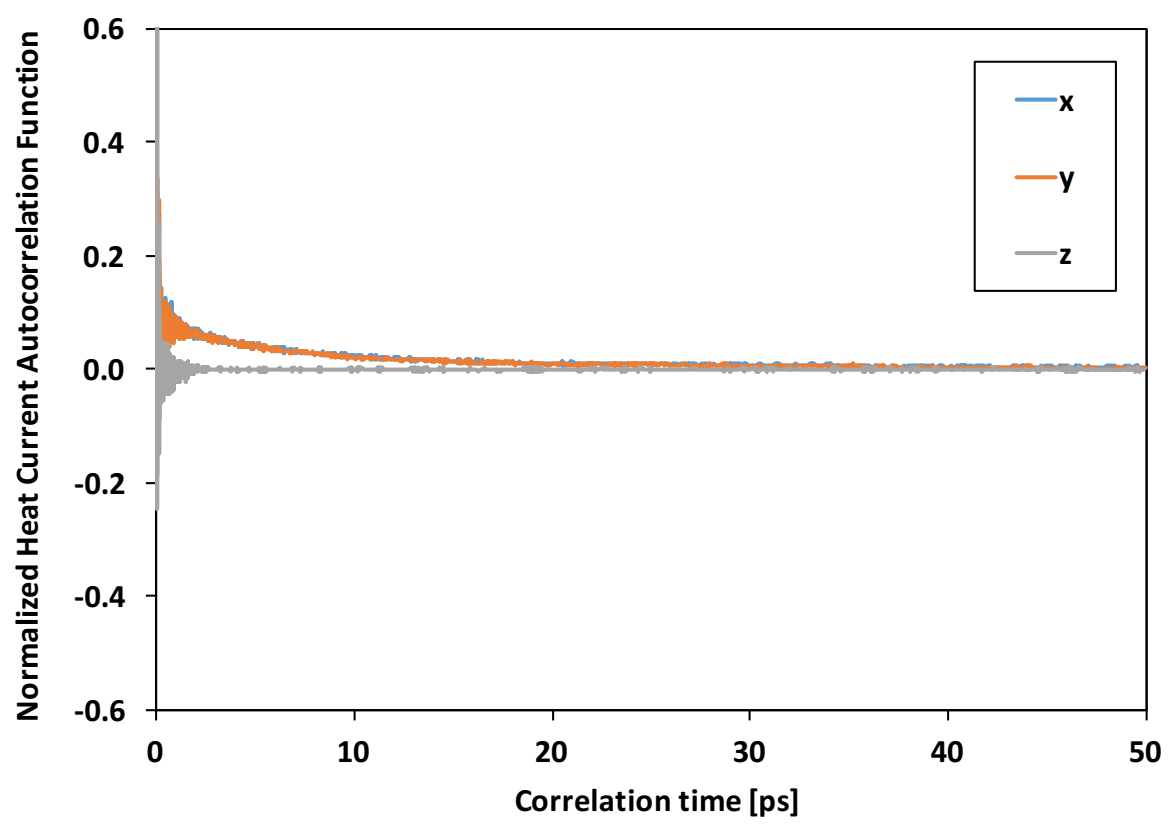

Figure 32. Normalized heat flux autocorrelation function for 3-layer GNP/CE system.

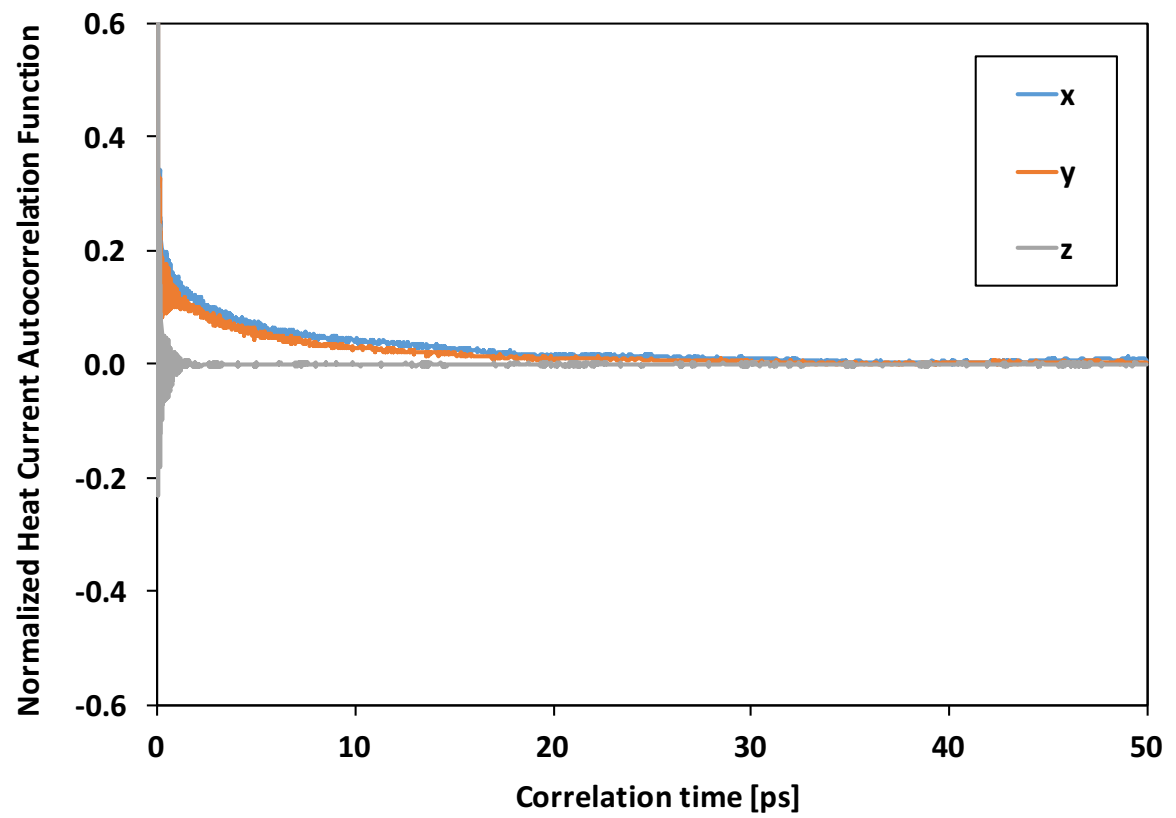

Figure 33. Normalized heat flux autocorrelation function for 4-layer GNP/CE system. 


\subsubsection{Micromechanics}

Micromechanics analysis code based on the generalized method of cells (MAC/GMC) was implemented to determine the effective thermal conductivity of GNP/CE nanocomposites and $\mathrm{GNP} / \mathrm{CF} / \mathrm{CE}$ hybrid composites.

To determine the effective thermal conductivity of randomly oriented GNP/CE nanocomposites, all MD thermal conductivities $\left(\kappa_{x}^{M D}, \kappa_{y}^{M D}\right.$, and $\left.\kappa_{z}^{M D}\right)$ were randomized by arithmetic averaging $[102,103]$ as shown in equation $(20)$. MAC/GMC was then used to determine the randomized thermal conductivity $\left(\kappa_{\text {random }}^{M D}\right)$ of GNP/CE nanocomposites of varying overall GNP concentrations ( $w_{G N P}^{R U C}$ ) from 1 to $8 \mathrm{wt} \%$ by $1 \mathrm{wt} \%$ increments. A triply periodic repeating unit cell (RUC) with finite filler in a square packing array $(A R C H I D=1)$ was applied by assigning the bulk thermal conductivity of neat $\mathrm{CE}$ as the matrix region and assigning the effective thermal conductivity of randomized GNP/CE as the filler region. The RUC is shown in Figure 27.

$$
\begin{gathered}
\kappa_{\text {random }}^{M D}=\frac{\kappa_{x}^{M D}+\kappa_{y}^{M D}+\kappa_{z}^{M D}}{3} \\
w_{M D}^{R U C}=\frac{w_{G N P}^{R U C}}{w_{G N P}^{M D}} \\
v_{M D}^{R U C}=\frac{\rho_{C E} w_{M D}^{R U C}}{\rho_{G N P / C E}+w_{M D}^{R U C}\left(\rho_{C E}-\rho_{G N P / C E}\right)}
\end{gathered}
$$

$\mathrm{MAC} / \mathrm{GMC}$ requires the volume fraction of the filler region which is given by the volume fraction of the GNP/CE MD cell in the RUC $\left(v_{M D}^{R U C}\right)$. The mass fraction of GNP in the RUC $\left(w_{G N P}^{R U C}\right)$ determines the mass fraction of the GNP/CE MD cell in the RUC $\left(w_{M D}^{R U C}\right)$ and can be calculated by using equation (21). The mass fraction of GNP in each MD system is a 
property of the MD model and is given in Table 10 . Then, $w_{G N P}^{R U C}$ was converted to $v_{M D}^{R U C}$ by using equation (22). The mass densities of neat $\mathrm{CE}\left(\rho_{C E}\right)$ and $\mathrm{GNP} / \mathrm{CE}\left(\rho_{G N P / C E}\right)$ systems are shown in Table 10. The aspect ratio of the filler region was set to 1 so that the effective thermal conductivity of GNP/CE was isotropic.

The effective thermal conductivities of GNP/CF/CE hybrid composites were also calculated by using MAC/GMC. This calculation used a circular fiber approximation RUC (rectagular pack or 'Architecture 13' as implemented in MAC/GMC). This RUC has $26 \times$ 26 subcells where the 312 middle subcells were assigned to fiber region and the other subcells were the matrix region. AS4 carbon fiber properties were used in the fiber region, possessing axial and transverse thermal conductivities of 6.50 and $2.18 \mathrm{~W} / \mathrm{mK}$, respectively [73]. The matrix region was applied the thermal conductivity of randomized GNP/CE (from the preceding MAC/GMC calculation) as the matrix of the hybrid composite. The volume fraction of the carbon fiber was 0.56 for all calculations. The aspect ratio $(\mathrm{R})$ of the $\mathrm{RUC}$, that is, the ratio between the $\mathrm{x}_{2}$ and $\mathrm{x}_{3}$ directions (see Figure 27), was set to 1 . Note that the mass fraction of GNP in the hybrid composites represented the amount of GNP in the matrix region only. The hybrid composite effective properties were determined in the axial (along the fiber) and transverse directions.

\subsection{Experimental fabrication and testing*}

For Neat CE samples [26]: A multi-part mold was coated with Mann Ease Release 300, then assembled and pre-heated in a vacuum oven at $100{ }^{\circ} \mathrm{C}$. The amount of Resin needed was weighed into a beaker, the corresponding amount of Hardener was weighed into the same beaker. The beaker was then placed under a Ross High Sear Mixer (HSM-100 LSKI) with 2" dispersion blade. The mixture was mixed at $1000 \mathrm{rpm}$ for $10 \mathrm{~min}$, until the two parts were thoroughly mixed. The mixture was then degassed at $100{ }^{\circ} \mathrm{C}$ and $29 \mathrm{in} \mathrm{Hg}$, alternating vacuum to avoid overflow, until no more bubbles appeared. The mixture was then poured into the pre-heated mold and degassed once more. The curing cycle used was

* This section was performed and wrote by Julie M Tomasi. 
$100{ }^{\circ} \mathrm{C}$ for 1 hour, then heated to $200{ }^{\circ} \mathrm{C}$ at $2^{\circ} \mathrm{C} / \mathrm{min}$ then held at $200{ }^{\circ} \mathrm{C}$ for two hours. The oven was then turned off and the cured epoxy was allowed to cool in the oven to room temperature, $\sim 1{ }^{\circ} \mathrm{C} / \mathrm{min}$ cooling rate.

For 4 wt\% GNP (Asbury Carbon TC307) in CE samples: A multi-part mold was coated with Mann Ease Release 300, then assembled and pre-heated in a vacuum oven at $100{ }^{\circ} \mathrm{C}$. The amount of TC307 GNP needed was weighed into a beaker, the corresponding amount of Hardener was weighed into the same beaker. The beaker was then placed under a Ross High Sear Mixer (HSM-100 LSKI) with 2" dispersion blade. The mixture was mixed at $3000 \mathrm{rpm}$ for 1 hour, then the mixture was placed in a Branson Sonicator CPX2800H operating at $40 \mathrm{kHz}$ and sonicated for 1 hour. The needed amount of Resin (mixed Resin and Hardener) was added to the mixture, then mixed at $1000 \mathrm{rpm}$ for $10 \mathrm{~min}$, until well mixed. The mixture was then degassed at $100{ }^{\circ} \mathrm{C}$ and 29 in $\mathrm{Hg}$, alternating vacuum to avoid overflow, until no more bubbles appeared. The mixture was then poured into the pre-heated mold and degassed once more. The curing cycle used was $100{ }^{\circ} \mathrm{C}$ for 1 hour, then heated to $200{ }^{\circ} \mathrm{C}$ at $2{ }^{\circ} \mathrm{C} / \mathrm{min}$ then held at $200{ }^{\circ} \mathrm{C}$ for two hours. The oven was then turned off and the cured epoxy was allowed to cool in the oven to room temperature, $\sim 1$ ${ }^{\circ} \mathrm{C} / \mathrm{min}$ cooling rate.

Through-plane thermal conductivity was measured using a Holometrix Model TCA-300 Thermal Conductivity Analyzer, which follows ASTM F433 [79] guarded heat flow meter method. The though-plane thermal conductivity of a $3.2 \mathrm{~mm}$ thick, $5 \mathrm{~cm}$ diameter discshaped test specimen was measured at $55^{\circ} \mathrm{C}$. Table 11 shows the through-plane thermal conductivity for neat $\mathrm{CE}$ and $4 \mathrm{wt} \% \mathrm{GNP} / \mathrm{CE}$ at $55^{\circ} \mathrm{C}$. The thermal conductivity increases slightly for the $4 \mathrm{wt} \% \mathrm{GNP} / \mathrm{CE}$ as compared to the neat $\mathrm{CE}$.

Table 11 Thermal conductivity of neat ce and $4 \mathrm{wt} \%$ gnp/ce from experiment.

\begin{tabular}{ccc}
\hline Samples & Number of Samples & Thermal Conductivity $(\mathrm{W} / \mathrm{mK})$ \\
\hline Neat CE & 4 & $0.150 \pm 0.002$ \\
$4 \mathrm{wt} \% \mathrm{GNP} / \mathrm{CE}$ & 5 & $0.178 \pm 0.002$ \\
\hline
\end{tabular}




\subsection{Results}

All GNP/CE molecular models have consistent density profiles along the z-direction (perpendicular to GNP) as shown from Figure 34 to Figure 37. The middle peaks represent the GNP layers and the plateaus on both sides indicate the cured CE regions. The crosslink percentage of the matrix in all nanocomposite models is about $80 \%$. The vertical dashed lines were drawn as boundaries to distinguish the GNP and matrix regions. These boundaries lines were determined by the van der Waals's radius of the carbon atoms in GNP which is $3.55 \AA$ according to the OPLS-AA force field. The GNP volume fraction of the MD system can be calculated from the ratio of the length of the GNP region to the overall length of the simulation box in the z-direction, since the $x-y$ area is the same throughout the simulation box. The mass densities of all MD models are shown in Table 10. The neat CE models have an average mass density equal to $1.199 \mathrm{~g} / \mathrm{cm}^{3}$. For the GNP/CE models, the average mass densities are equal to $1.243,1.330,1.401$, and 1.498 $\mathrm{g} / \mathrm{cm}^{3}$ for 1, 2, 3, and 4 layer(s) of GNP nanocomposite models, respectively.

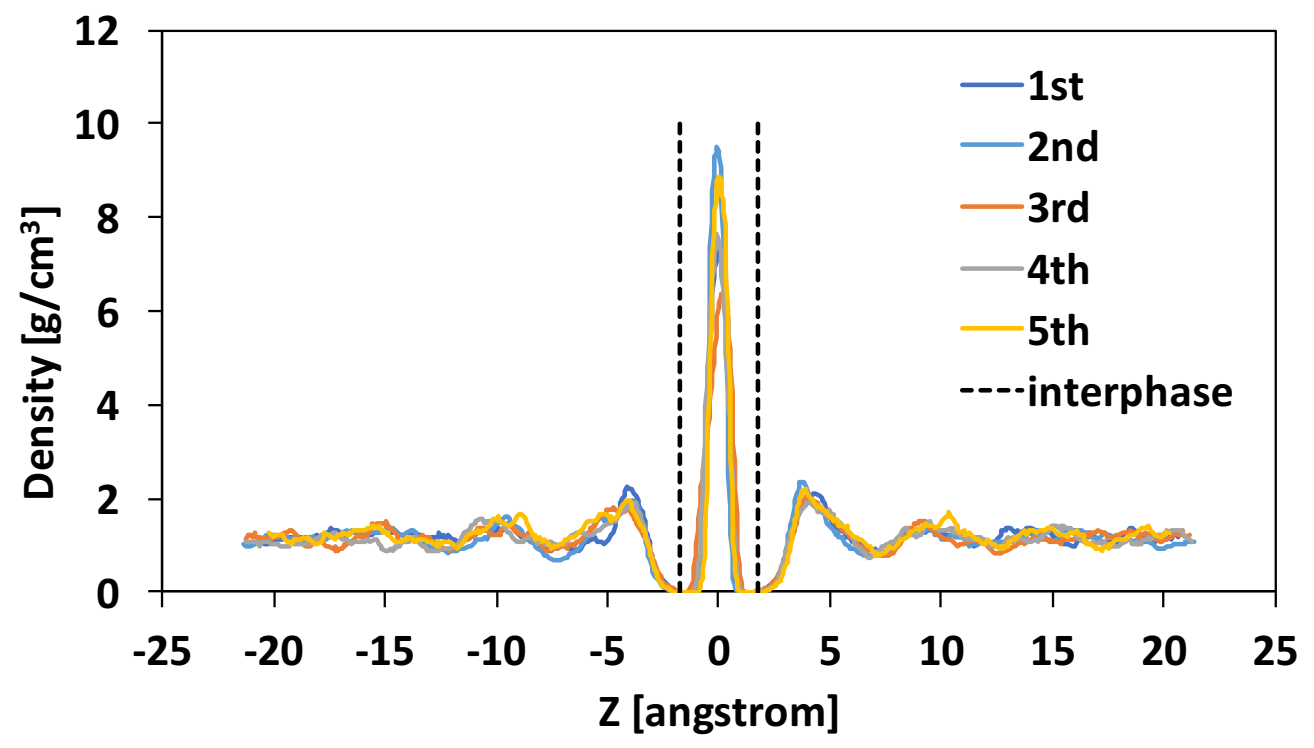

Figure 34. Plot of density profiles of the equilibrated models of 1-layer GNP/CE system. 


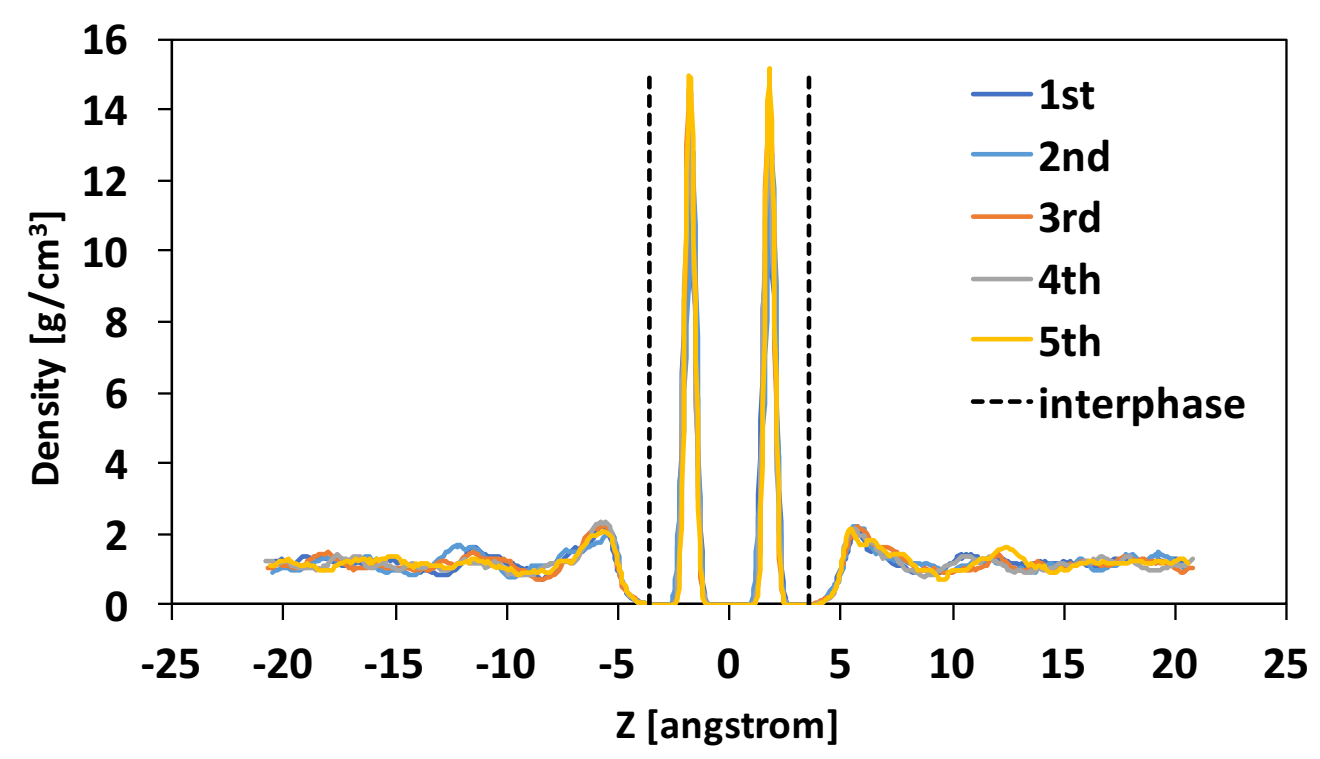

Figure 35. Plot of density profiles of the equilibrated models of 2-layer GPN/CE system.

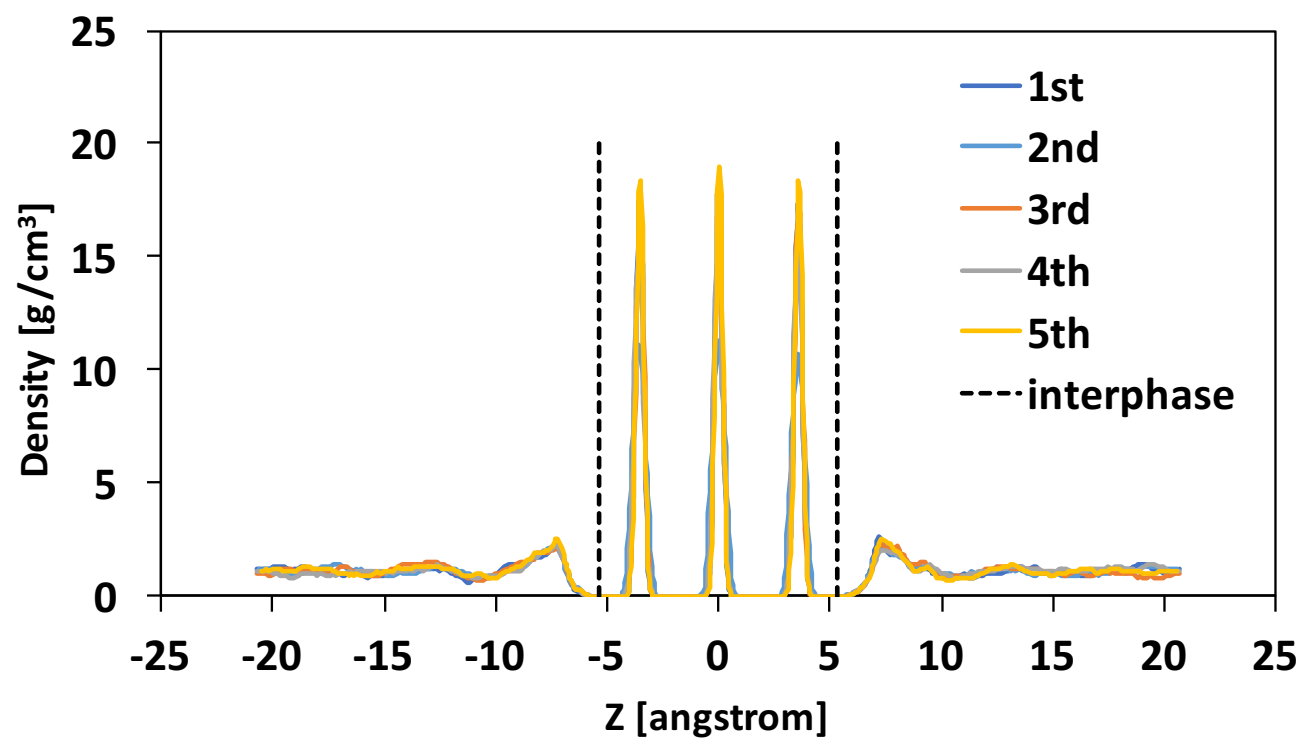

Figure 36. Plot of density profiles of the equilibrated models of 3-layer GNP/CE system. 


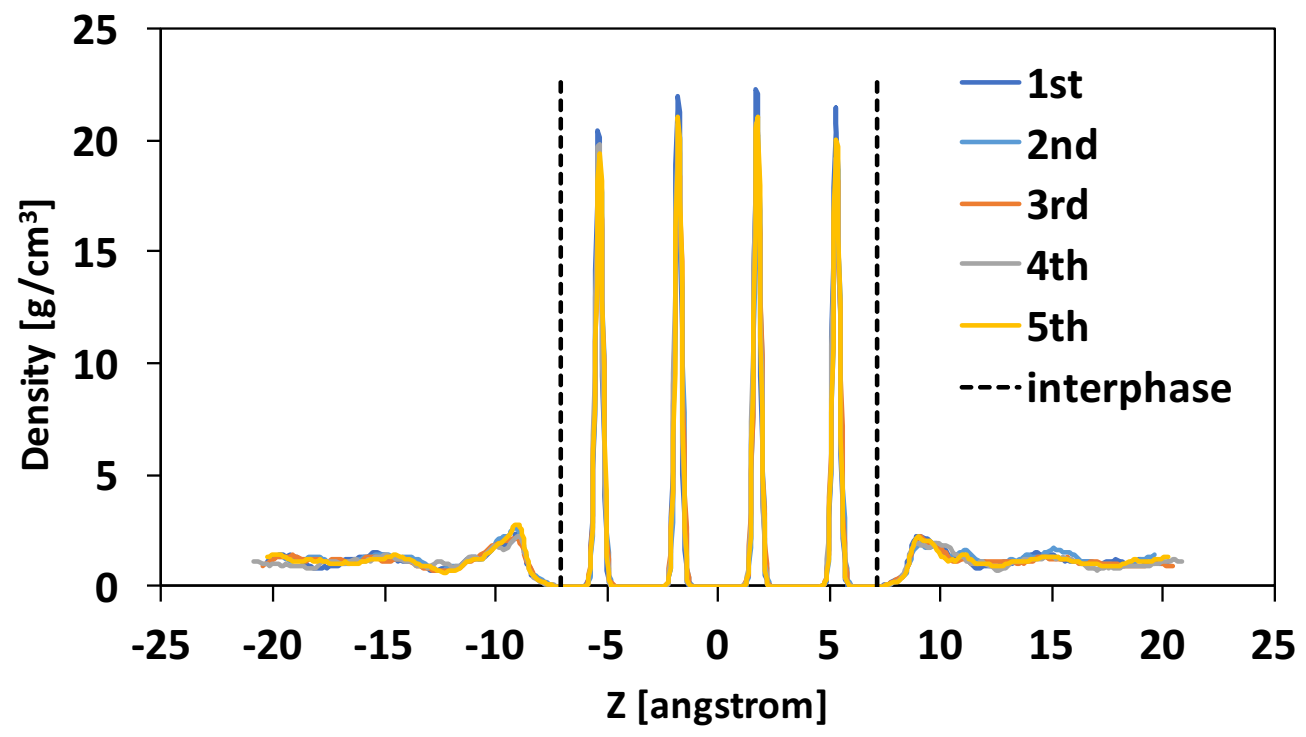

Figure 37. Plot of density profiles of the equilibrated models of 4-layer GNP/CE system.

From Table 10, the in-plane (parallel) thermal conductivities (prior to randomization) obtained from MD of CE nanocomposites with 1, 2, 3 and 4-layer GNP are equal to 98.0, $215.4,317.3$, and $464.4 \mathrm{~W} / \mathrm{mK}$ with $0.151,0.287,0.408$, and 0.517 of GNP mass fraction, respectively. The GNP volume fractions of these four systems are equal to $0.085,0.172$, 0.258 , and 0.349 , respectively. The out-of-plane (perpendicular) thermal conductivities are equal to $0.192,0.184,0.171$, and $0.151 \mathrm{~W} / \mathrm{mK}$, respectively, which decrease with more layers of GNP. However, the overall thermal conductivities will primarily be affected by the in-plane conductivities.

In this chapter, the normalized thermal conductivity was calculated as the ratio between the effective thermal conductivity of the models with and without GNP. The normalized thermal conductivity was used to determine how GNPs improve the thermal conductivity of composite materials. In Figure 38, the normalized thermal conductivity of randomized $1 \mathrm{GNP} / \mathrm{CE}$ (blue line) is the highest compared to the other systems at the same GNP concentrations. It is clear that better GNP dispersion gives higher thermal conductivity. At $1 \mathrm{wt} \% \mathrm{GNP}$ of $1 \mathrm{GNP} / \mathrm{CE}$, the normalized thermal conductivity of our prediction and the experimental measurement of GNP/Epoxy from Kostagiannakopoulou et al. [17] are equal 
to 1.11 , and 1.10, respectively, which shows good agreement. The model predicts a lower value when compared with the GNP/Epoxy nanocomposite specimen from Yang et al. [28] which resulted in a normalized conductivity of 1.24 for $1 \mathrm{wt} \%$ GNP. The normalized thermal conductivity of the $3 \mathrm{wt} \%$ GNP specimen of Kostagiannakopoulou et al. [17] is equal to 1.41 , comparing well with the model (1.46). However, the $5 \mathrm{wt} \% 1 \mathrm{GNP} / \mathrm{CE}$ model is $20 \%$ higher than the experimental specimen. While the $1 \mathrm{GNP} / \mathrm{CE}$ model and Kostagiannakopoulou et al. data agree well for low GNP concentration, the model diverges from the experimental measurement for high $w t \%$ GNP. The observed discrepancy is likely due to the increased difficulty of achieving good GNP dispersion at greater loadings. Therefore, we estimate that the specimen from Kostagiannakopoulou et al. can theoretically be further increased in conductivity by $20 \%$ with improved dispersion.

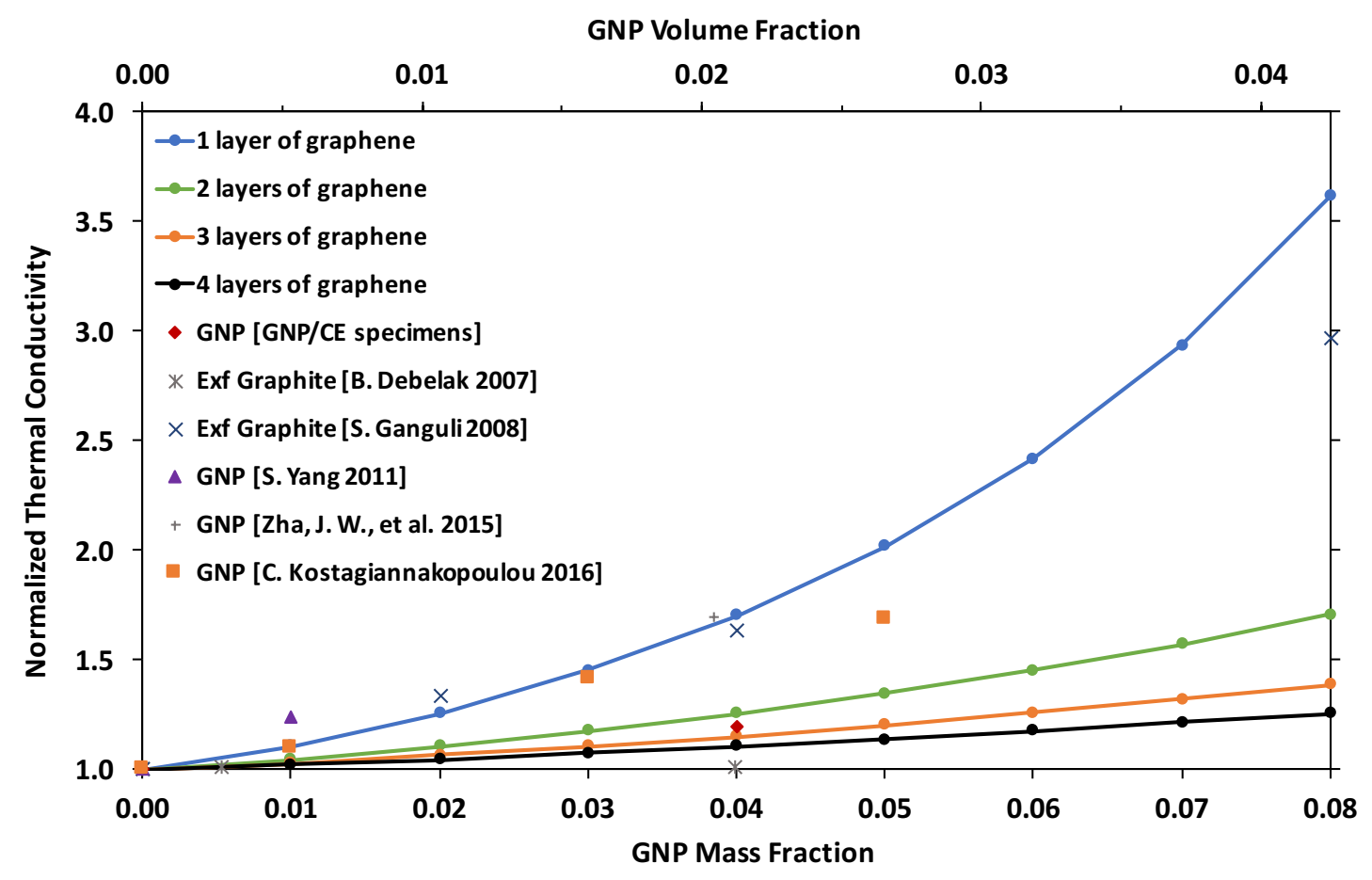

Figure 38. Plot of normalized thermal conductivity of randomized GNP/CE models as function of GNP mass fraction compared with experiments [11, 14, 17, 28]. 
Similar behavior is also observed when comparing the 1GNP/CE model with the values of GNP/Epoxy obtained by Ganguli et al. The normalized thermal conductivities of 1CE/GNP at $2 \mathrm{wt} \%$ and $4 \mathrm{wt} \%$ GNP are 1.26 and 1.71, respectively, and are in good agreement with exfoliated graphite specimens from Ganguli et al. [14] yielding values of 1.33 and 1.64, respectively. At $8 \mathrm{wt} \% \mathrm{GNP}, 1 \mathrm{GNP} / \mathrm{CE}$ has a higher normalized thermal conductivity than the same GNP mass fraction specimen of Ganguli et al. [14]. For this loading, the predicted and experimental values are 3.63and 2.96, respectively. This shows that the experimental specimen likely has GNP agglomeration for high GNP concentrations. Ganguli et al. also observed that there was the optimum GNP concentration before the GPN re-agglomerated when having the higher GNP concentration. In this research, we experimentally measured the normalized thermal conductivity of $4 \mathrm{wt} \% \mathrm{GNP} / \mathrm{CE}$ to be 1.19 . Our experimental GNP/CE specimen falls between the double-layer GNP and triple-layer GNP models. While there is probably significant variation in the number of GNP layers in the experimental specimen, our multiscale model suggests that the GNP is effectively two-tothree layers thick. The exfoliated GNP/Epoxy specimens from Debelak and Lafdi resulted in normalized thermal conductivities lower than the $4 \mathrm{GNP} / \mathrm{CE}$ model for both $0.5 \%$ and 4 wt $\%$ GNP, meaning that the specimens could have more than four layers of exfoliated graphite.

The effective thermal conductivities of $\mathrm{GNP} / \mathrm{CF} / \mathrm{CE}$ hybrid composite were also normalized with the $\mathrm{CF} / \mathrm{CE}$ composite (without GNP) for both the axial and transverse directions (shown in Figure 39 and Figure 40). Both plots show that improving the GNP dispersion results in higher thermal conductivity. The axial thermal conductivity of the $\mathrm{GNP} / \mathrm{CF} / \mathrm{CE}$ hybrid composite increases less than $8 \%$ for $8 \mathrm{wt} \%$ of single layer GNP and less than $2 \%$ for $8 \mathrm{wt} \%$ of non-single layer GNP. By doping GNP into the composites, the thermal conductivity hardly increases in the fiber direction because the axial thermal conductivity of the carbon fiber dominates this property. 


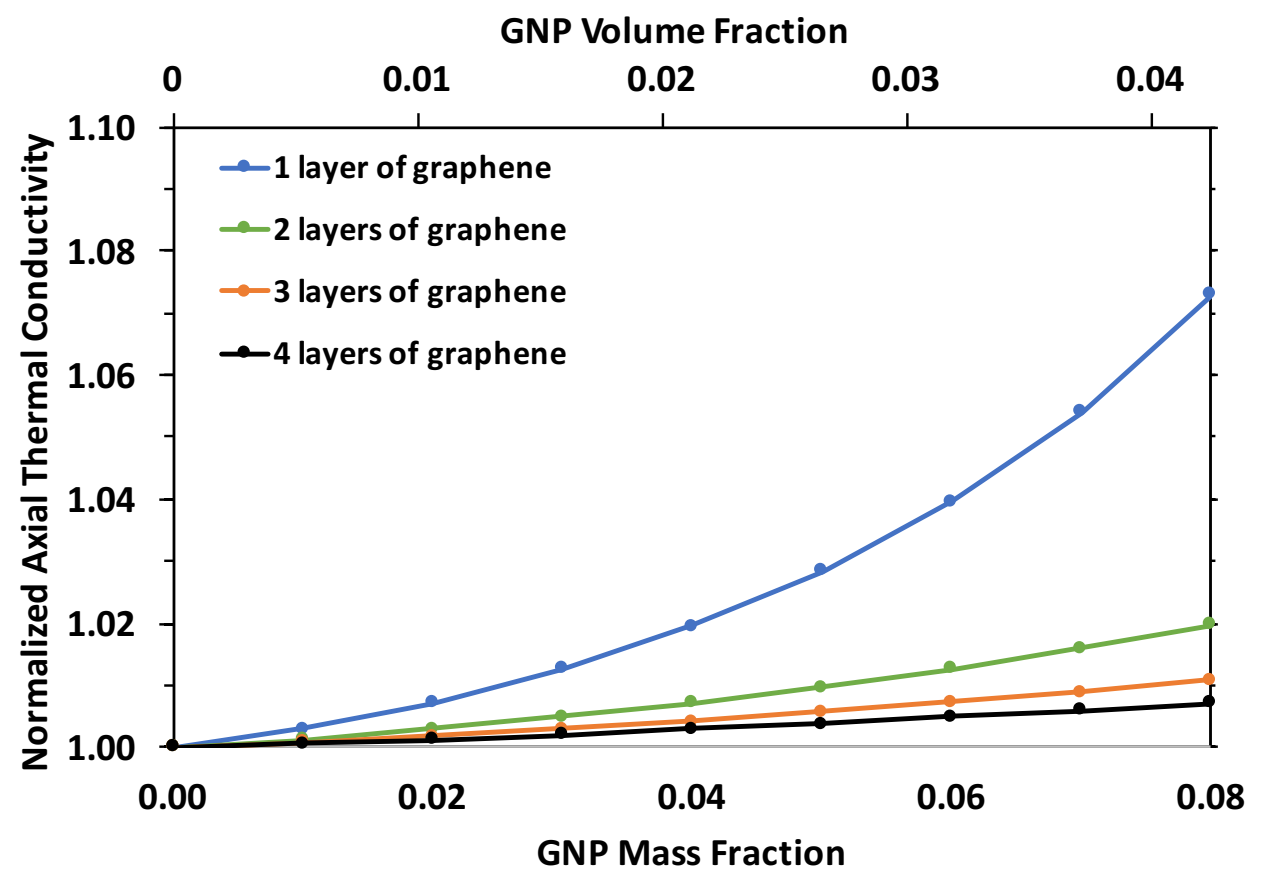

Figure 39. Plot of normalized axial thermal conductivity of GNP/CF/CE hybrid composites models.

The transverse thermal conductivity of the GNP/CF/CE hybrid composites model is greatly improved by doping GNP. At $8 \mathrm{wt} \%$ single-layer GNP, the transverse thermal conductivity is predicted to increase by $132 \%$ when compared with no GNP reinforcement. For the same GNP mass fraction of 2, 3, and 4-layer GNP, the hybrid composite transverse thermal conductivities can be raised up to $47 \%, 27 \%$, and $18 \%$ when compared with the absence of GNP.

In Figure 40, the results from the multiscale model of $\mathrm{GNP} / \mathrm{CF} / \mathrm{CE}$ are compared with the experimental values of GNP/CF/Epoxy. The normalized thermal conductivity through the composite plane (transverse) measured by Zhang et al. [31] are equal to 1.11 and 1.45 for $0.5 \%$ and $4 \mathrm{wt} \%$, respectively, of pristine graphene (to the epoxy matrix). For $0.5 \mathrm{wt} \%$ single-layer GNP in the GNP/CF/CE hybrid composites, the model underestimates the conductivity, but the $4 \mathrm{wt} \%$ single-layer GNP model estimated the normalized transverse thermal conductivity to be 1.47 , which is very close to the experiment value. These specimens are conjectured to have well-dispersed pristine graphene. 


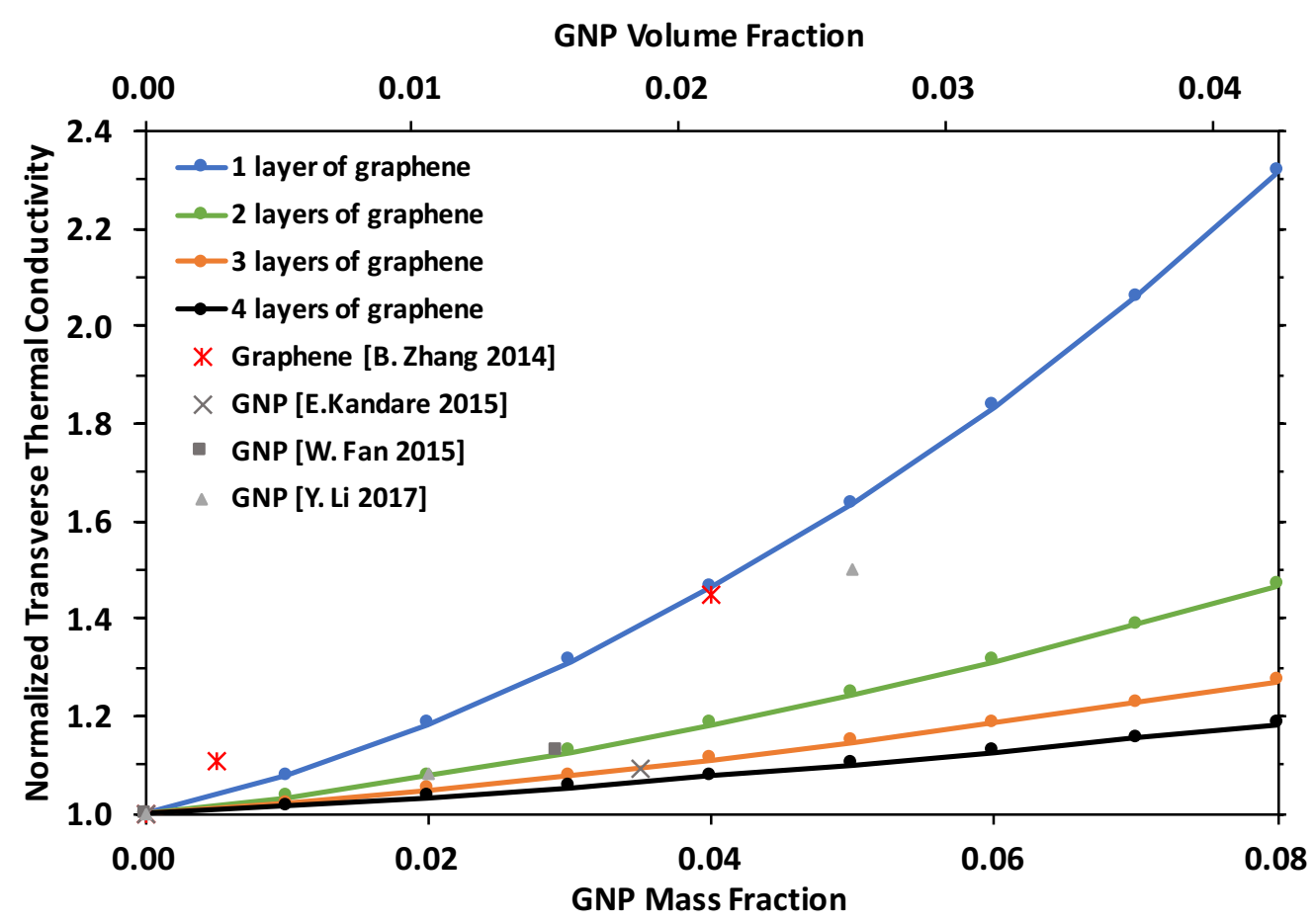

Figure 40. Plot of normalized transverse thermal conductivity of GNP/CF/CE hybrid composites models compared with experiments $[13,16,18,31]$.

Fan et al. [13] examined the transverse thermal conductivity of GNP/CF/Epoxy hybrid composites with $1 \mathrm{wt} \%$ of GNP (to the overall composite with $55 \%$ of carbon fiber or 2.9 wt\% GNP relative to matrix) equal to 1.13 times the composites without GNP. By comparison with our multiscale model, this experimental specimen could have effectively double-layered GNP. Additional GNP/CF/epoxy hybrid composites specimens were investigated by Kandare et al. [16] with 1 vol\% of GNP (to the overall composite with 45 vol\% of carbon fiber). The hybrid composite specimen resulted in an increase of $9 \%$ in the transverse thermal conductivity when compared with no GNP reinforcement. By comparison with our model, this hybrid composite could have effectively triple layers of GNP. Another experimental study was conducted by Li et al. [18] where GNP/CF/epoxy hybrid composites were created with $2 \%$ and $5 \mathrm{wt} \%$ of GNP (to the epoxy matrix). Their transverse thermal conductivities improved $8 \%$ and $50 \%$ compared to the carbon fiber reinforced plastic without GNP. The $2 \mathrm{wt} \%$ GNP specimen appears to possibly be double- 
layered GNP, and the $5 \mathrm{wt} \%$ GNP specimen falls between our single- and double-layered GNP models.

The GNP can improve the thermal conductivity in the transverse direction because the transverse thermal conductivity of carbon fiber is lower than the axial direction and the matrix usually plays the dominant role in the transverse properties. Consequentially, if the thermal conductivity of the matrix increases, the effective thermal conductivity of hybrid composite also improves. Moreover, the improvement of thermal conductivity in transverse direction actually improves on two axes in Cartesian coordinate system.

\subsection{Conclusions}

A multiscale modeling approach was developed to predict the thermal conductivity of CEmatrix hybrid composites using EMD with OPLS-AA force field and micromechanics. Our method provides realistic results for the thermal conductivity of GNP/CE nanocomposites and $\mathrm{GNP} / \mathrm{CF} / \mathrm{CE}$ hybrid composites when compared with experiments. The perfectly dispersed GNP model gives the highest thermal conductivity of our GNP/CE systems. For low GNP concentrations, several experimental results closely match the perfectly dispersed model, but none of these experimental specimens were observed to reach the ideal model for $5 \mathrm{wt} \% \mathrm{GNP}$ or greater. Thus, we suspect that some agglomeration occurs for these highly loaded specimens and improvements in thermal conductivity can theoretically be achieved by pursuing even better dispersion. Our results provide a guide for other researchers to assess the effective dispersion of $\mathrm{GNP} / \mathrm{CE}$ and $\mathrm{GNP} / \mathrm{CF} / \mathrm{CE}$ experimental samples as we have demonstrated here. For GNP/CF/CE hybrid composites, the transverse thermal conductivity is predicted to increase up to $132 \%$ for $8 \mathrm{wt} \%$ GNP (compared to no GNP) given ideal dispersion. 


\section{CHAPTER 5 MULTISCALE MODELING: THERMAL CONDUCTIVITY OF CARBOXYL ( $-\mathrm{COOH}$ ) FUNCTIONALIZED GRAPHENE NANOPLATELET/CYCLOALIPHATIC EPOXY NANOCOMPOSITE}

\subsubsection{Introduction}

The thermal transport is extremely important to the advance applications such as the electronic industry, spacecraft, and aircraft. This is because the heat is always generated during the operation so the heat dissipation from the heat source is required to let those applications work effectively and safely. As the superior thermal conductivity of pristine graphene, the experimental value can be above $5,000 \mathrm{~W} / \mathrm{mK}$ at the room temperature $[5$, 6]. The graphene nanoplatelet (GNP) with perfect dispersion which can improve the thermal conductivity of epoxy is desired. One of the technique that can facilitate the dispersion of GNP is the functionalization [104].

In this chapter, Cycloaliphatic Epoxy (CE) and Anhydride Curing Agent (ACA) are both used to study the effect of the functionalized GNP. Since curing mechanism between CE and ACA has a carboxyl and hydroxyl groups as reactive groups, either one of these groups is selected to be the functionalized group. Recently, MD work [105] shows that the functionalized group can reduce the interfacial resistance between graphene and paraffin. The out-of-plane thermal conductivity can be potentially increased by adding the functionalized groups to GNP. Moreover, this study also shows that the carboxyl group is better than the hydroxyl in term of the interfacial resistant reduction. Therefore, the carboxyl group is focused to study the effect of GNP functionalization (fGNP) on thermal conductivity of fGNP/CE composites. 
The objective of this study is to understand how the covalently functionalization between GNP and epoxy affect the thermal conductivity. A single-layer GNP is focused in this study and functionalized with carboxyl group. Multiscale modeling is used to study the fGNP/CF/CE composites model including Molecular Dynamics (MD) modeling and micromechanics. The thermal conductivity of fGNP/crosslinked CE nanocomposites is determined by Equilibrium MD with different amount of functionalization. The thermal conductivities are homogenized by arithmetic average and scaled the GNP mass fraction by micromechanics.

\subsection{Computational Details}

The carboxyl fGNP models were prepared by using MD. Then, each fGNP model was combined with neat $\mathrm{CE}$ system to determine the thermal conductivity of $\mathrm{fGNP} / \mathrm{CE}$ nanocomposites by using EMD. At microscale, the thermal conductivity of nanocomposite was randomized by arithmetic average and scaled the GNP mass fraction by using micromechanical modeling. All details of each step are given as following.

\subsubsection{MD modeling}

\subsubsection{Functionalized Graphene MD Model}

The single-layer graphene with 576 carbon atoms was prepared as shown in Figure 41 by using "lattice" command in LAMMPS. For the functional group, the carboxyl groups were separately created with two different formations as shown in Figure 42. These representative carboxyl groups were used to randomly add above and below the GNP layer by using Python script. The script generated 100 small boxes spanned on the top and bottom of GNP simulation box by offsetting $4 \AA$ from the edge of GNP simulation box. Then, each carboxyl group was put into the small box with the same number of groups on each side. There were two different amount of carboxyl groups: 5 and 10 carboxyl groups on each side which were called 5-5fGNP and 10-10fGNP, respectively as shown in Figure 43. 


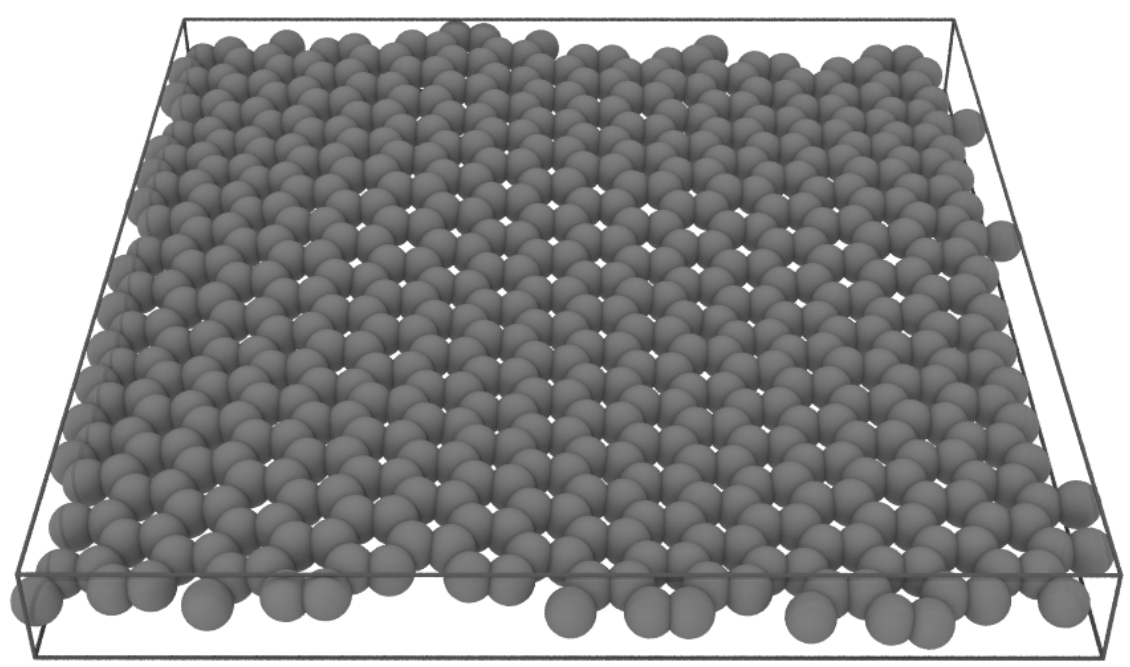

Figure 41 A single-layer GNP model for preparing fGNP model.
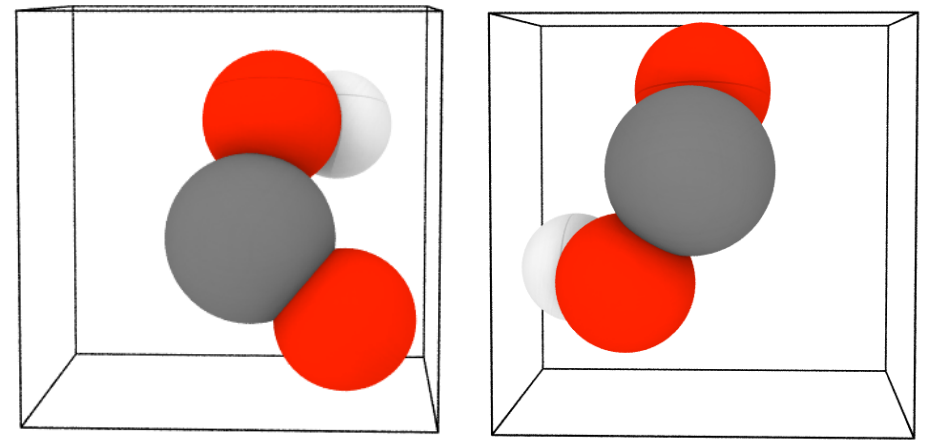

Figure 42 The molecular structures of carboxyl groups for placing above (left) and below (right) GNP, respectively. Red: oxygen, gray: carbon, white: hydrogen.
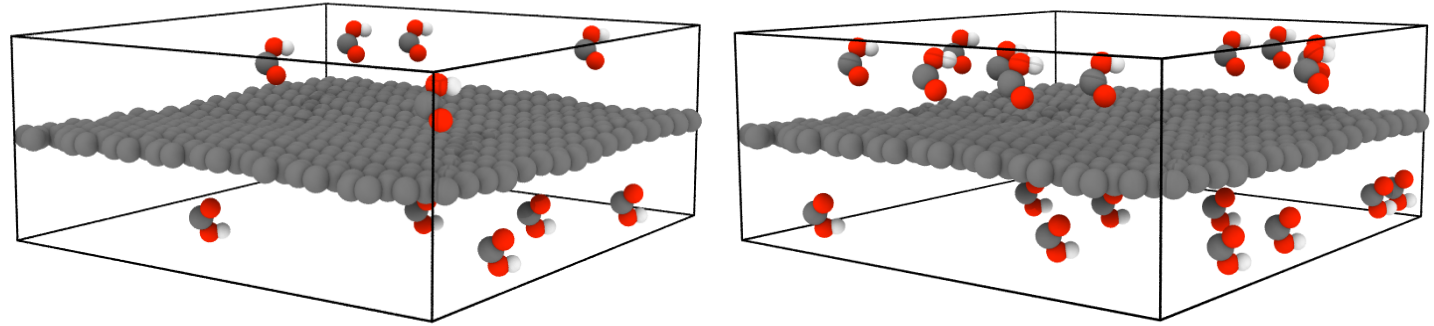

Figure 43 Representative pre-functionalized GNP and carboxyl groups models for 5-5fGNP/CE (left) and $10-10 \mathrm{GNP} / \mathrm{CE}$ (right) systems. 
The combined fGNP model was created the bond between carbon atoms from a carboxyl and GNP by using "fix bond/create" command on LAMMPS. The atom type of functionalized carbon on GNP was changed from $\mathrm{sp}^{2}$ to be $\mathrm{sp}^{3}$ type. All angles and dihedrals were updated by using Python script. The bonded fGNP models were minimized energy using "minimize" command with conjugate gradient (CG) algorithm by setting 10e4 energy tolerance and then dynamically equilibrated using NPT ensemble for 300 ps with 1 fs time steps at 300K. Figure 44 and Figure 45 shows the molecular structure of equilibrated 5-5fGNP and 10-10fGNP models, respectively.

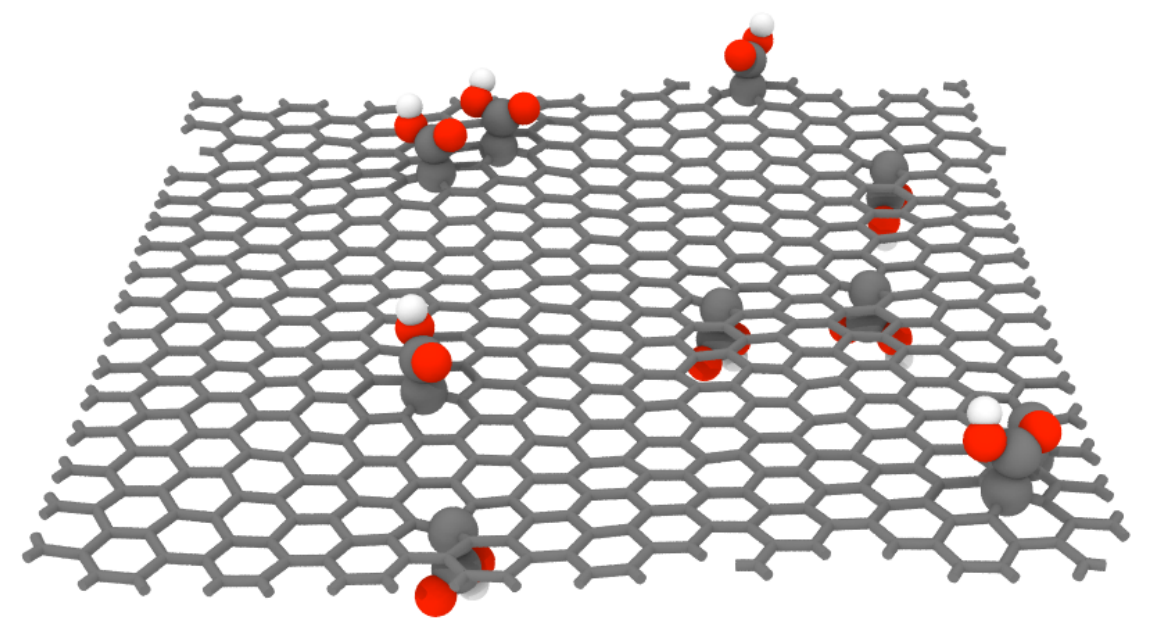

Figure 44 Molecular structure of 5-5fGNP.

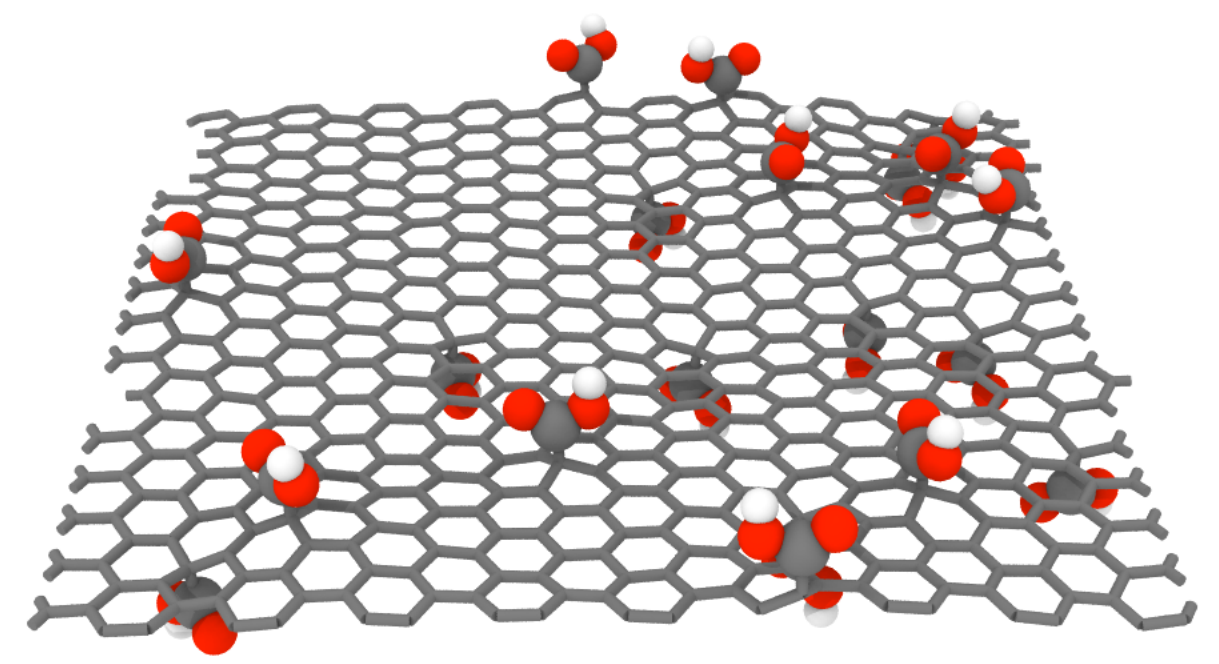

Figure 45 Molecular structure of 10-10fGNP. 
Figure 46 The ratio between defected carbon $\left(\mathrm{SP}^{3}\right)$ and GNP carbon $\left(\mathrm{SP}^{2}\right)$ on fGNP

\begin{tabular}{cc}
\hline Model & $\mathrm{SP}^{3} / \mathrm{SP}^{2}$ of carbon (\%) \\
\hline $5-5 \mathrm{fGNP} / \mathrm{CE}$ & 1.74 \\
$10-10 \mathrm{fGNP} / \mathrm{CE}$ & 3.47 \\
\hline
\end{tabular}

\subsubsection{2 fGNP/CE nanocomposites MD Model}

The nanocomposite model of fGNP/CE includes fGNP model as the nanoparticle shown in Figure 44 and Figure 45 and CE system as the matrix. Epoxy Cyclohexyl Methyl 3,4 Epoxy Cyclohexyl Carboxylate (EEC) and 4, 7-Methanoisobenzofuran-1, 3-dione, 3a, 7, 7a-tetrahydromethyl or Anhydride Curing Agent (ACA) are the resin and hardener of CE matrix, respectively. In this MD model, the modified ACA also included to initiate the curing mechanism. Molar ratio of EEC : ACA : modified ACA was equal to $7: 13: 1$ with total 5,373 CE atoms.

All fGNP, EEC, ACA, and modified ACA molecules were combined into a long box which normal direction of fGNP was in $\mathrm{z}$ direction and the plane stayed in middle of simulation box. The box was compressed by using "fix deform" command in LAMMPS and NVT ensemble was used to control the temperature at $300 \mathrm{~K}$. The final dimensions were 38.69, $37.70,36.18 \AA$ in $\mathrm{x}, \mathrm{y}, \mathrm{z}$ direction, respectively. This densification ran for total $5.2 \mathrm{~ns}$ with $1 \mathrm{fs}$ time steps using the stepwise approach shown in Table 9. Lennard-Jones cutoff radius was $10 \AA$. Density profile on each direction was determine at the end of simulation to ensure that the density profiles of system were uniform. Five models of 5-5fGNP/CE and 10-10fGNP/CE models were created to obtain the good-statistical results.

The crosslinking process had two part. The first part was to establish the crosslinked bond between carboxyl group on fGNP and epoxide group on EEC similar to the esterification reaction. The initial bond between oxygen atom on carboxyl group and carbon atom on epoxide group was created by using "fix bond/create" command with $7 \AA$ as minimum 
distance in LAMMPS. The simulation performed by NVT ensemble for $200 \mathrm{fs}$ with $0.1 \mathrm{fs}$ time steps at $300 \mathrm{~K}$. Non-bonded interaction was Lennard-Jones with $15 \AA$ cutoff radius to prevent any high energy atom moves away. Then, the related bonds were updated by using Python script. All angle and dihedral were also reassigned by using general angle-dihedral Python script. The hydroxyl groups were appeared after the reaction.

The second part was the crosslinking process in CE matrix. There were two reactions that were esterification and anhydride ring opening reaction. For the esterification, the carboxyl groups on modified ACA molecules were first reacted with the epoxide groups on EEC molecules by using "fix bond create" command with similar control as mentioned above. The relative bond and VdW configuration was updated by Python script giving hydroxyl groups. Their angle and dihedral information was reassigned by general angle-dihedral Python script. The hydroxyl groups from the functionalization and the esterification were consumed to open anhydride ring on ACA molecules.

For the anhydride ring opening reaction, the initial bonds between hydrogen atom and oxygen atom were created by using "fix bond/create" command which those atoms were from hydroxyl group and anhydride ring, respectively. The bonds were built with the same previous bond-created setup. Another bonds related to the initial bond were generated by running Python script. The relative bonds on the carboxyl groups and the anhydride rings were assigned to be deleted within the same Python script. For relative angle and dihedral, the general angle-dihedral Python script updated according to the new bonds. This reaction exposed the carboxyl groups which were continuously used in the esterification. Both reactions alternately performed till $80 \%$ of the crosslink percentage was achieved.

After the crosslinking process, the crosslinked models were equilibrated by using NPT ensemble with at $1 \mathrm{~atm}$ and $300 \mathrm{~K}$. The barostat was chosen to be "aniso" type. LennardJones potential was used as non-bonded interaction with $10 \AA$ cutoff radius. The mass density of fGNP/CE model was averaged for last 12 ps of simulation. The density profile along out-of-plane direction of fGNP was determined with 1,000 flattened slabs using "fix ave/chunk" command. The density profile was determined by averaging the mass density 
each slab every 1 ps using 10 input with interval of 100 time steps. The simulations were performed along $2 \mathrm{~ns}$ with 1 fs time steps. The average mass densities and GNP volume fractions of both systems are shown in Table 12. The equilibrated 5-5fGNP/CE and 1010fGNP/CE models shown as Figure 47 and Figure 48 were ready to determine the thermal conductivity.

Table 12 Density, GNP volume fraction, and thermal conductivity of fGNP/CE, GNP/CE and neat CE at $300 \mathrm{~K}$.

\begin{tabular}{|c|c|c|c|c|}
\hline \multirow{2}{*}{ Model } & \multirow{2}{*}{$\begin{array}{l}\text { GNP volume } \\
\text { fraction }\end{array}$} & \multirow{2}{*}{ Density $\left(\mathrm{g} / \mathrm{cm}^{3}\right)$} & \multicolumn{2}{|c|}{ Thermal Conductivity (W/mK) } \\
\hline & & & In-plane & Out-of-plane \\
\hline neat $\mathrm{CE}$ & 0.000 & $1.199 \pm 0.006$ & \multicolumn{2}{|c|}{$0.237 \pm 0.016$} \\
\hline $1 \mathrm{GNP} / \mathrm{CE}$ & 0.085 & $1.243 \pm 0.025$ & $98.019 \pm 11.560$ & $0.192 \pm 0.026$ \\
\hline 5-5fGNP/CE & 0.086 & $1.282 \pm 0.009$ & $6.662 \pm 0.938$ & $0.221 \pm 0.031$ \\
\hline 10-10fGNP/CE & 0.086 & $1.293 \pm 0.017$ & $4.574 \pm 1.041$ & $0.218 \pm 0.020$ \\
\hline
\end{tabular}

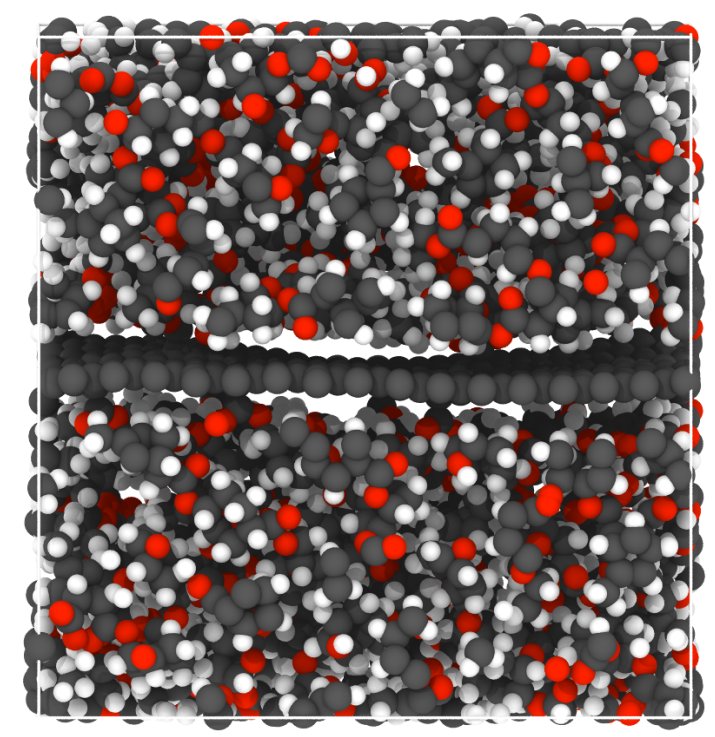

Figure 47 Representative crosslinked model of 5-5fGNP/CE after equilibration. 


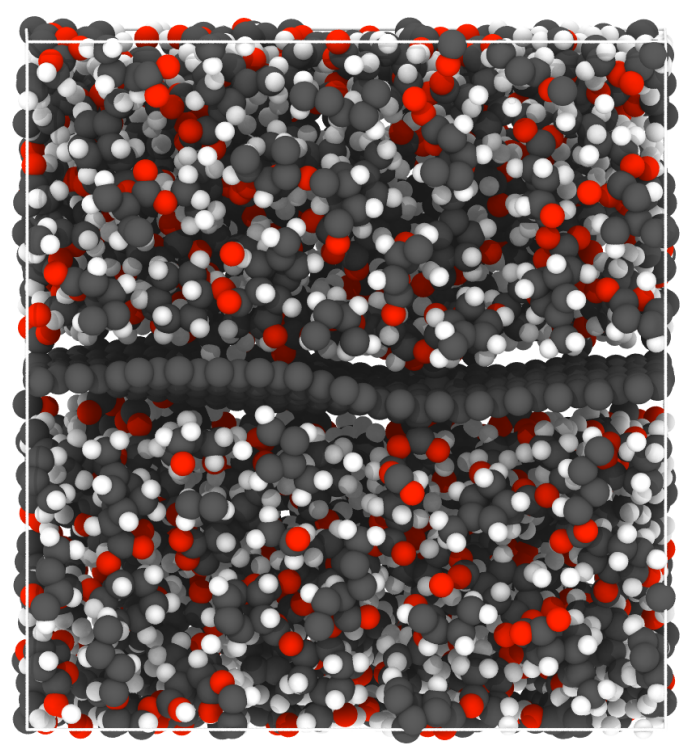

Figure 48 Representative crosslinked model of 10-10fGNP/CE after equilibration.

\subsubsection{Thermal conductivity by EMD}

EMD method based on Green-Kubo approach was used to find the thermal conductivity of fGNP/CE nanocomposites. NVT ensemble perform for $10 \mathrm{ps}$ with $1 \mathrm{fs}$ time steps to obtain system temperature at $300 \mathrm{~K}$. The heat flux was then computed from kinetic energy, potential energy, and stress by using "compute stress/atom".

$$
\kappa_{x}=\frac{1}{\mathrm{k}_{\mathrm{B}} \mathrm{T}^{2} V} \int_{0}^{\infty}\left\langle\mathrm{J}_{\mathrm{x}}(0) \mathrm{J}_{\mathrm{x}}(\mathrm{t})\right\rangle \mathrm{dt}
$$

The heat flux (J) was identified main signal by using autocorrelation function to conceal the noise in heat flux data. The autocorrelation function was performed by using "fix ave/correlate" command with "auto" option. This command auto correlated the heat flux total 10,000 inputs with interval of 5 time steps and total correlation time was $50 \mathrm{ps}$. This heat flux autocorrelation function (HFACF) was integrated by using trapezoidal rule. The thermal conductivity was calculated form equation (23) which the integral term was divided by Boltzmann constant, Square of temperature, and system volume. The thermal conductivities of 5-5fGNP/CE and 10-10fGNP/CE models are shown in Table 12. 


\subsubsection{Micromechanics}

Micromechanics method of fGNP/CE composites was similar to the micromechanics calculation of GNP/CE composites in Chapter 4 . A $2 \times 2 \times 2$ Repeating Unit Cell (RUC) was used in the same manner. A subcell was applied the randomization fGNP/CE's thermal conductivity which averaged the thermal conductivities in all axes from MD models shown in Table 12. The thermal conductivity of neat CE was added into the others seven subcells in the RUC. The aspect ratio of 1 was chosen in this calculation.

\subsection{Results}

Table 13 shows the results of the covalently functionalization between fGNP with carboxyl group and CE matrix for two levels of functionalization. The 5-5fGNP/CE and 10$10 \mathrm{fGNP} / \mathrm{CE}$ systems can potentially have 1.7 and $3.5 \%$ of functionalization, respectively. During the functionalization process between carboxyl groups on fGNP and epoxide groups on EEC, the 5-5fGNP/CE models are $100 \%$ of potential functionalization but the 10-10 fGNP/CE models are about $90 \%$ of potential functionalization. Therefore, the actual functionalization of 5-5fGNP/CE and $10-10 \mathrm{fGNP} / \mathrm{CE}$ is 1.7 and $3.1 \%$ of functionalization.

Table 13 Functionality and crosslink density of fGNP/CE nanocomposites models.

\begin{tabular}{ccccc}
\hline \multirow{3}{*}{ Model } & \multicolumn{3}{c}{ fGNP/CE } & Crosslink density \\
& $\begin{array}{c}\text { Potential } \\
\text { functionality } \\
(\%)\end{array}$ & $\begin{array}{c}\text { Percentage of covalent bond } \\
\text { between fGNP and CE } \\
(\%)\end{array}$ & $\begin{array}{c}\text { Actual } \\
\text { functionality } \\
(\%)\end{array}$ & $\begin{array}{c}\text { in matrix } \\
(\%)\end{array}$ \\
\hline 5-5fGNP/CE & 1.7 & 100 & 1.7 & 72 \\
$10-10 \mathrm{fGNP} / \mathrm{CE}$ & 3.5 & 90 & 3.1 & 67 \\
\hline
\end{tabular}

During the crosslink process, some of epoxide groups were already consumed by the functionalization. The crosslink densities of CE matrix in 5-5fGNP/CE and 10-10fGNP/CE systems are shown in Table 13 which are lower than the crosslink of the CE matrix in 
$1 \mathrm{GNP} / \mathrm{CE}$ and neat CE models. Figure 25 shows that the thermal conductivity by the EMD method has similar values for the systems with $60-90 \%$ crosslink.

Figure 49 shows that the density profiles of 5-5fGNP/CE models have more fluctuation of the $\mathrm{CE}$ matrix regions compared to the density profiles of $1 \mathrm{GNP} / \mathrm{CE}$ models shown in Figure 34. This is because the fGNP surfaces are bumpy unlike the pristine graphene surface due to the inclusion of functionalized groups. Figure 49 also shows the planes of fGNP are still flat and the density of fGNP is relatively uniform when comparing each model.

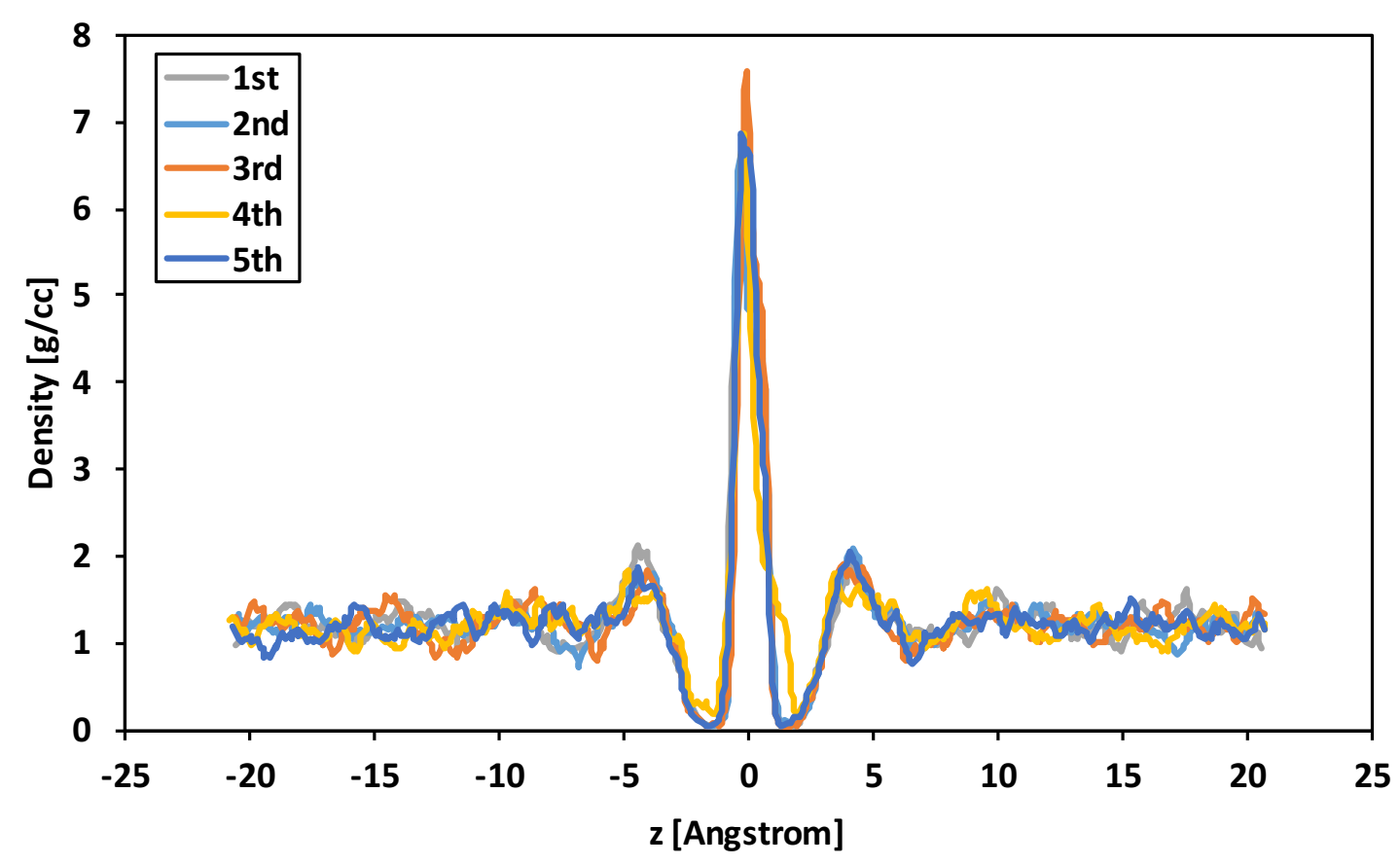

Figure 49 The plot of density profiles of 5-5fGNP/CE models along $\mathrm{z}$ axis.

For the 10-10fGNP/CE system, the density fluctuations are also found in the CE matrix regions shown in Figure 50. The density at interphase regions of this system have more oscillated than the 5-5fGNP/CE system because 10-10fGNP/CE system has more covalently bonded at the interphase region. Moreover, Figure 50 also shows that each density profile of fGNP is different from the others. The more covalently bonded functionalization can affect the plane of fGNP. The $3^{\text {rd }}$ model of 10-10fGNP/CE has two peaks of density profile in the fGNP region which is so different from the other model. 


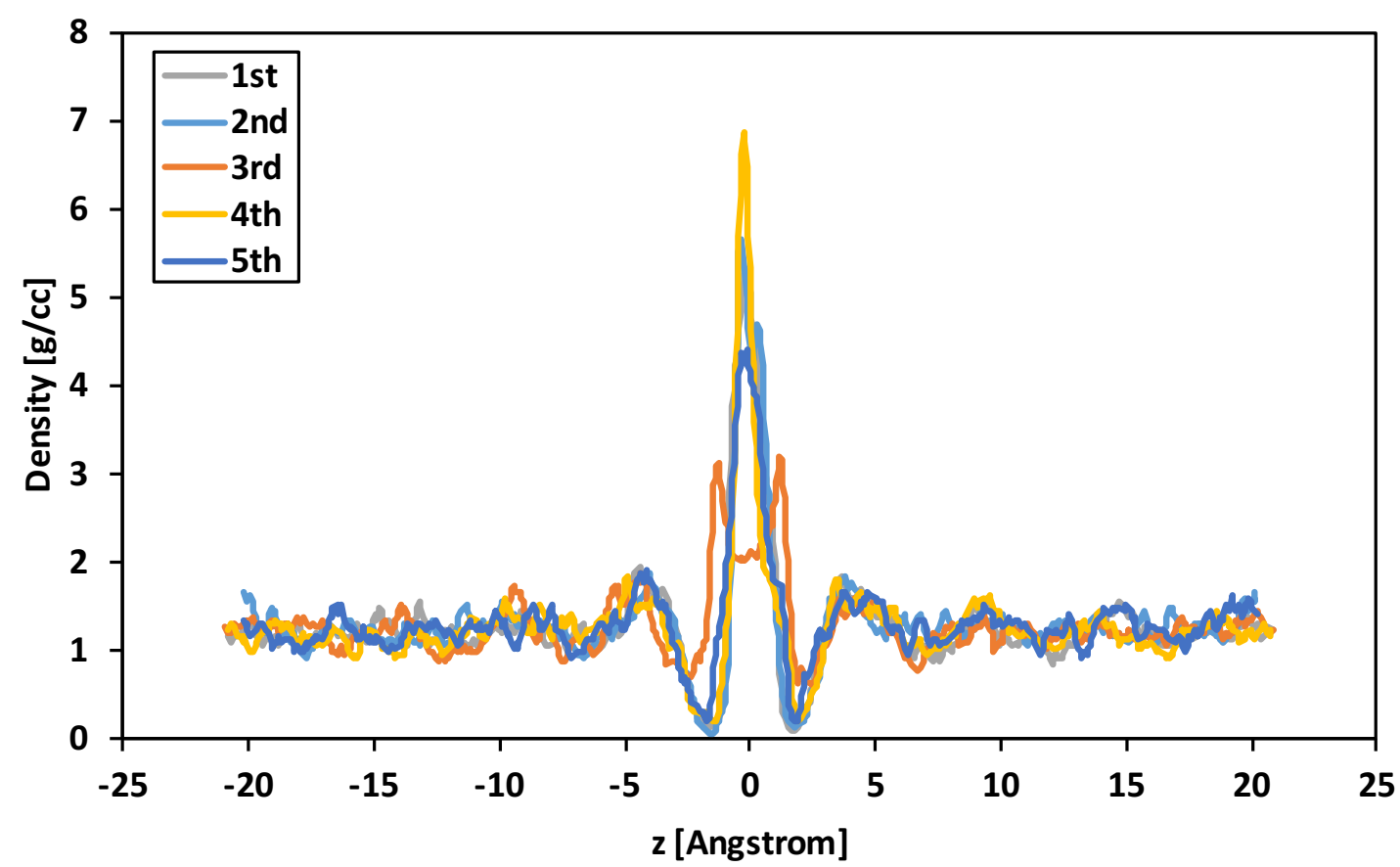

Figure 50 The plot of density profiles of 10-10fGNP/CE models along $\mathrm{z}$ axis.

The front view of the $3^{\text {rd }}$ fGNP/CE model is shown in Figure 51 which the fGNP is bent by the functionalized bonds. A particular cluster of carboxyl groups (red circle) on the bottom surface of fGNP shown in Figure 52 has effect on the shape of fGNP. On the top surface, the carboxyl group are located as surrounding the cluster. After the equilibration, these carboxyl groups which covalently bonded to the CE matrix are pulled by the expanding of CE matrix. However, the thermal conductivity of this particular model is relatively close to the other models. 


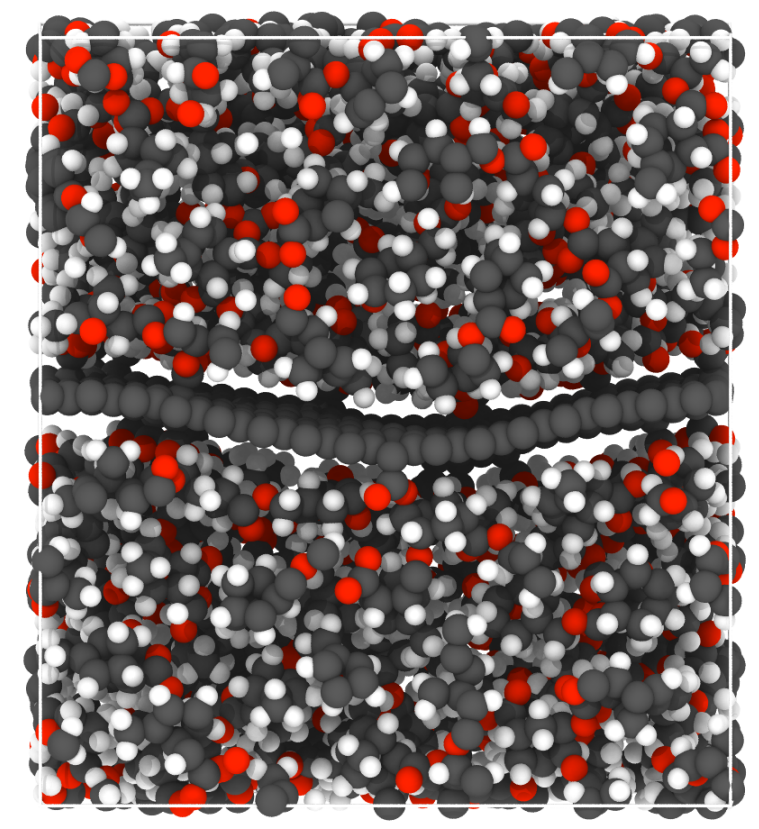

Figure 51 The $3^{\text {rd }}$ crosslinked model of $10-10 \mathrm{fGNP} / \mathrm{CE}$.
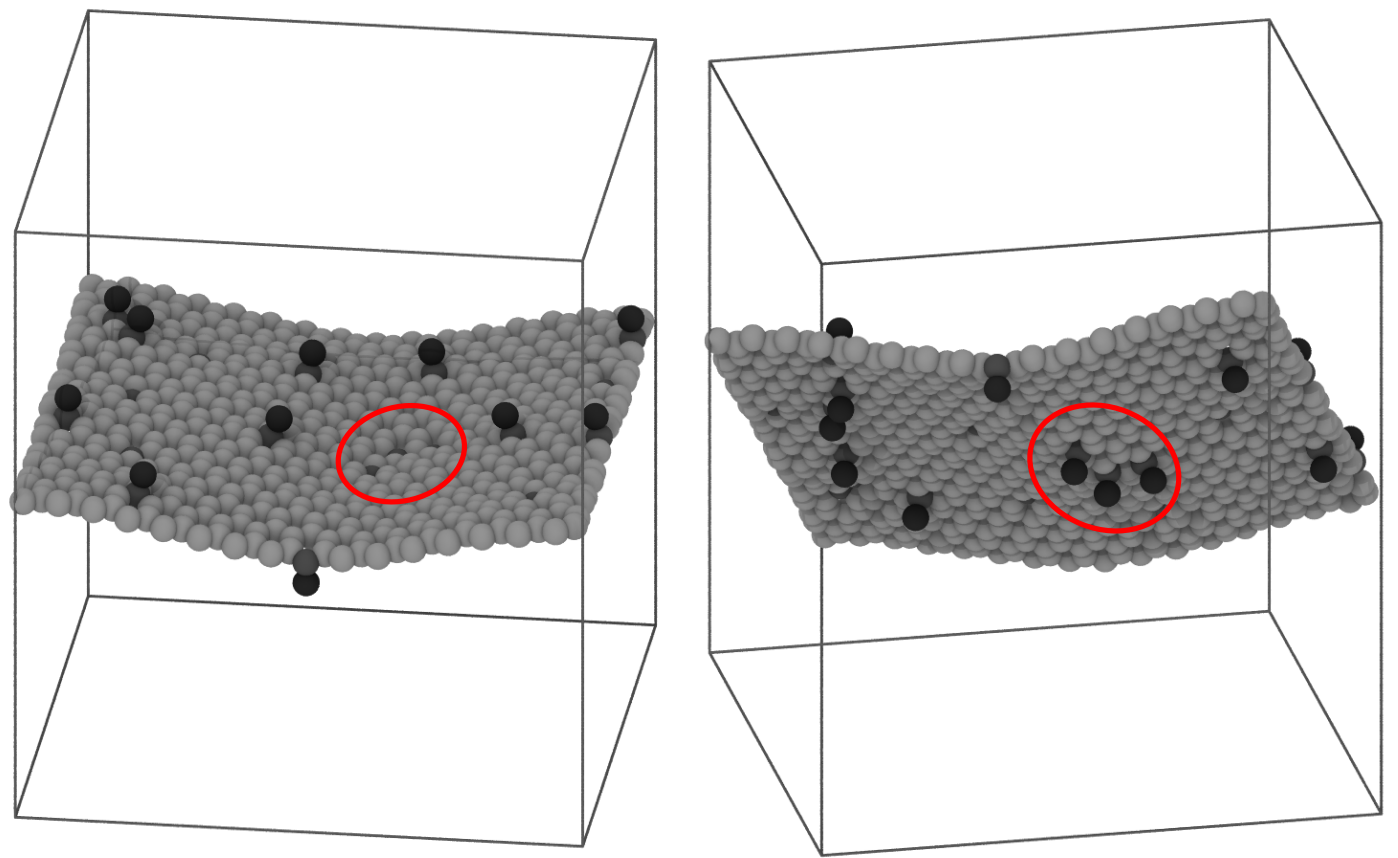

Figure 52 The cluster of functional groups shown on the top (left) and bottom (right) surfaces of fGNP of the $3^{\text {rd }} 10-10 \mathrm{fGNP} / \mathrm{CE}$ model. 
The thermal conductivities of 5-5fGNP/CE and 10-10fGNP/CE models are shown in Table 12. The in-plane thermal conductivities of these two systems are significant lower than the thermal conductivity of $1 \mathrm{GNP} / \mathrm{CE}$ systems. The phonon can easily travel through the crystal structure like graphene, however, the inclusion of functionalized groups disturbs the $\mathrm{sp}^{2}$ structure. Consequently, the more of defects on GNP, the lower in-plane thermal conductivity of nanocomposites can be obtained. The out-of-plane thermal conductivities of both fGNP/CE systems are higher than the 1GNP/CE system but they are still lower than neat CE system.

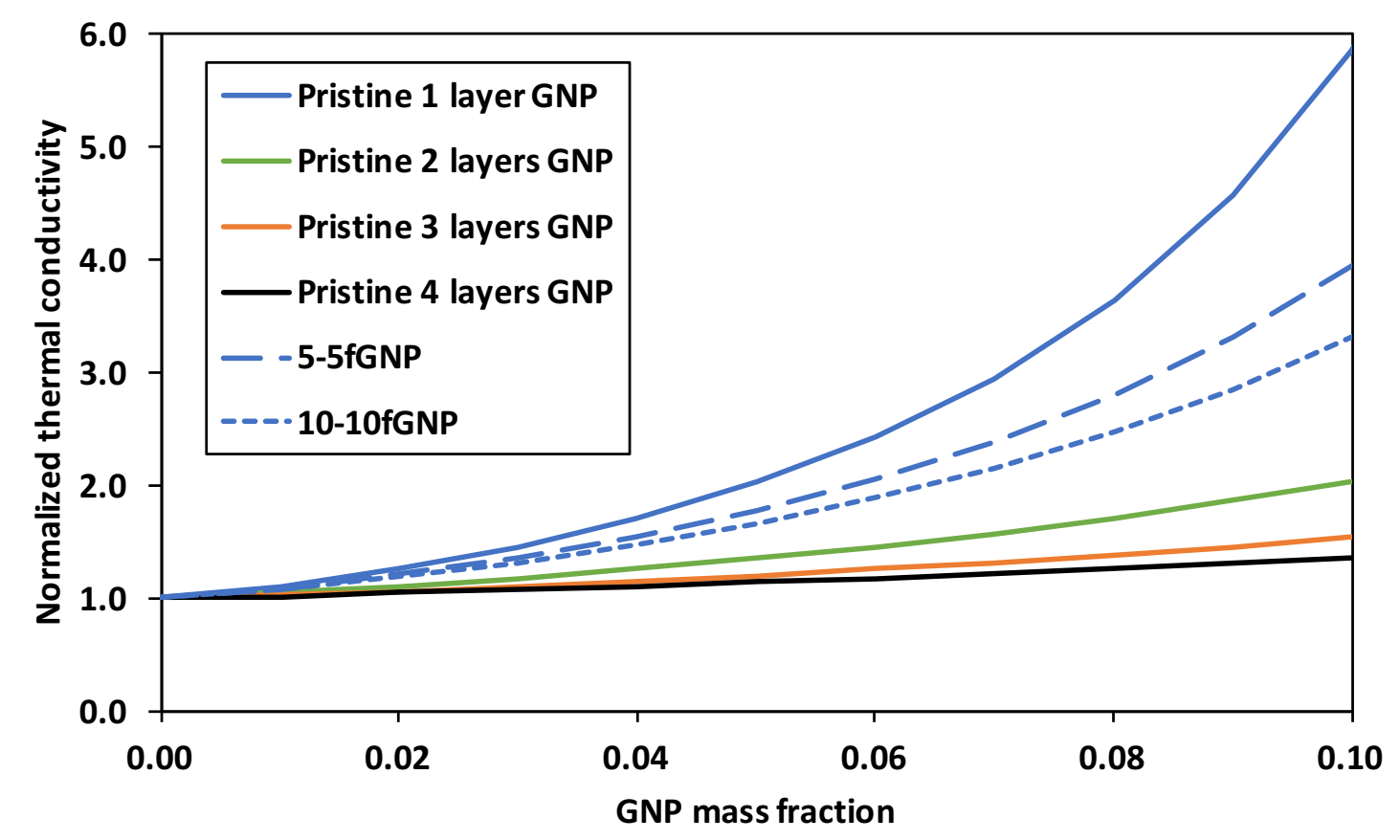

Figure 53 The plot of normalized thermal conductivity of functionalized and pristine GNP/CE as the function of GNP mass fraction.

Figure 53 shows the thermal conductivities of fGNP/CE composite models from $\mathrm{MAC} / \mathrm{GMC}$ as the function of GNP mass fraction. The plot shows the normalized thermal conductivities of 5-5fGNP/CE and 10-10fGNP/CE systems compared with the pristine GNP/CE models. The thermal conductivities (solid lines) of GNP/CE with four different levels of GNP dispersion are taken from Figure 38. The thermal conductivities (Dashed blue lines) of fGNP/CE are lower than the thermal conductivity (Solid blue line) of singlelayered pristine GNP/CE. The increasing of functionalization in fGNP/CE system 
decreases the thermal conductivity at the same level of GNP dispersion. However, both fGNP/CE systems have the higher thermal conductivities than the pristine GNP/CE system having more than one layer of GNP. The functionalization on GNP [106] can provide the better GNP dispersion to the composite. This effect can be also found in the single-wall carbon nanotube (CNT)/epoxy composites [107] which the functionalization improves CNT dispersion in epoxy composites.

\subsection{Conclusions}

The functionalized GNP/CE models can be established by using MD modeling and the EMD approach is able to predict the thermal conductivity fGNP/CE nanocomposites. The functionalization on GNP significantly decreases the in-plane thermal conductivity of GNP/CE nanocomposites. The out-of-plane thermal conductivity slightly increases due to the functionalization but no greater the thermal conductivity of neat CE.

Micromechanical calculation is capable to determine the thermal conductivity of fGNP/CE composites with different GNP mass fractions. The effective thermal conductivity of fGNP/CE decreases with increased the functionalized percentage. One of important aspect of functionalized GNP is to improve GNP dispersion in the composites. The thermal conductivities of fGNP/CE models are all higher than the thermal conductivities of 2-, 3-, and 4-layered GNP/CE models. Additionally, the aspect ratio of GNP and thermal pathway are still needed to be further investigated. 


\section{CHAPTER 6 MULTISCALE MODELING: COEFFICIENT OF LINEAR THERMAL EXPANSION OF CARBON FIBER/GRAPHENE NANOPLATELET/EPON862 HYBRID COMPOSITES}

\subsection{Introduction}

Carbon fiber (CF) composites with EPON862 matrix have been used in the structural components of aircraft and spacecraft. These composites are light, strong and chemically resistant. However, the stiffness of the composites in the transverse direction is weak due to the characteristic of matrix (EPON862 system). Graphene nanoplatelet (GNP) has been used as the additive to improve the mechanical properties of the matrix.

The prediction of mechanical properties of GNP/EPON862 nanocomposites. was studied by Hadden et al. (2015) [36]. Molecular dynamics (MD) was used along with OPLS all atom force field which the resulting elastic properties are shown in Table 14. Four different levels of GNP dispersion were investigated which a single layer GNP/EPON862 was determined as the perfectly dispersed system.

Table 14 Elastic properties of GNP/EPON862 models from Hadden et al. [36].

\begin{tabular}{ccccccccccccccc}
\hline $\begin{array}{c}\mathrm{GNP} \\
\text { layers }\end{array}$ & $\begin{array}{c}\mathrm{GNP} \\
\text { volume } \\
\text { fraction }\end{array}$ & $\mathrm{E}_{\mathrm{x}}$ & $\mathrm{E}_{\mathrm{y}}$ & $\mathrm{E}_{\mathrm{z}}$ & $\mathrm{G}_{\mathrm{xy}}$ & $\mathrm{G}_{\mathrm{xz}}$ & $\mathrm{G}_{\mathrm{yz}}$ & $\mathrm{V}_{\mathrm{xy}}$ & $\mathrm{V}_{\mathrm{xz}}$ & $\mathrm{V}_{\mathrm{yz}}$ & $\mathrm{V}_{\mathrm{yx}}$ & $\mathrm{V}_{z x}$ & $\mathrm{~V}_{z y}$ \\
\hline 1 & 0.111 & 93.4 & 94.8 & 2.432 & 0.243 & 0.001 & 0.001 & 0.13 & 0.46 & 0.471 & 0.158 & 0.109 & 0.025 \\
2 & 0.187 & 174.6 & 172.5 & 2.731 & 0.424 & 0.001 & 0.001 & 0.168 & 0.437 & 0.524 & 0.153 & 0.056 & 0.015 \\
3 & 0.271 & 239.4 & 238.3 & 3.005 & 0.582 & 0.001 & 0.001 & 0.152 & 0.44 & 0.446 & 0.151 & 0.01 & 0.034 \\
4 & 0.33 & 293.1 & 295.5 & 3.251 & 0.725 & 0.001 & 0.001 & 0.159 & 0.455 & 0.452 & 0.156 & 0.011 & 0.009 \\
\hline
\end{tabular}

The coefficient of linear thermal expansion (CLTE) is another crucial material property to indicate how the size of system changes along each Cartesian direction when the 
temperature increases or decreases. The CLTEs of GNP/EPON862 have not yet been studied by MD modeling. In this chapter, the CLTEs of GNP/EPON862 models were determined by MD. The CLTEs were homogenized for GNP/EPON862 composites and CF/GNP/EPON862 hybrid composites by micromechanics.

\subsection{Computational Details}

The work flow to determine effective CLTEs of CF/GNP/EPON862 hybrid composites is shown in Schematic 2. There were two main parts including MD modeling and micromechanics. For MD modeling, the MD models of GNP/EPON862 obtained from C. Hadden et al. (2015) [36] were determined the CLTE. For micromechanics, the effective CLTEs of GNP/EPON862 composites and CF/GNP/EPON862 hybrid composites were calculated including the GNP randomization.

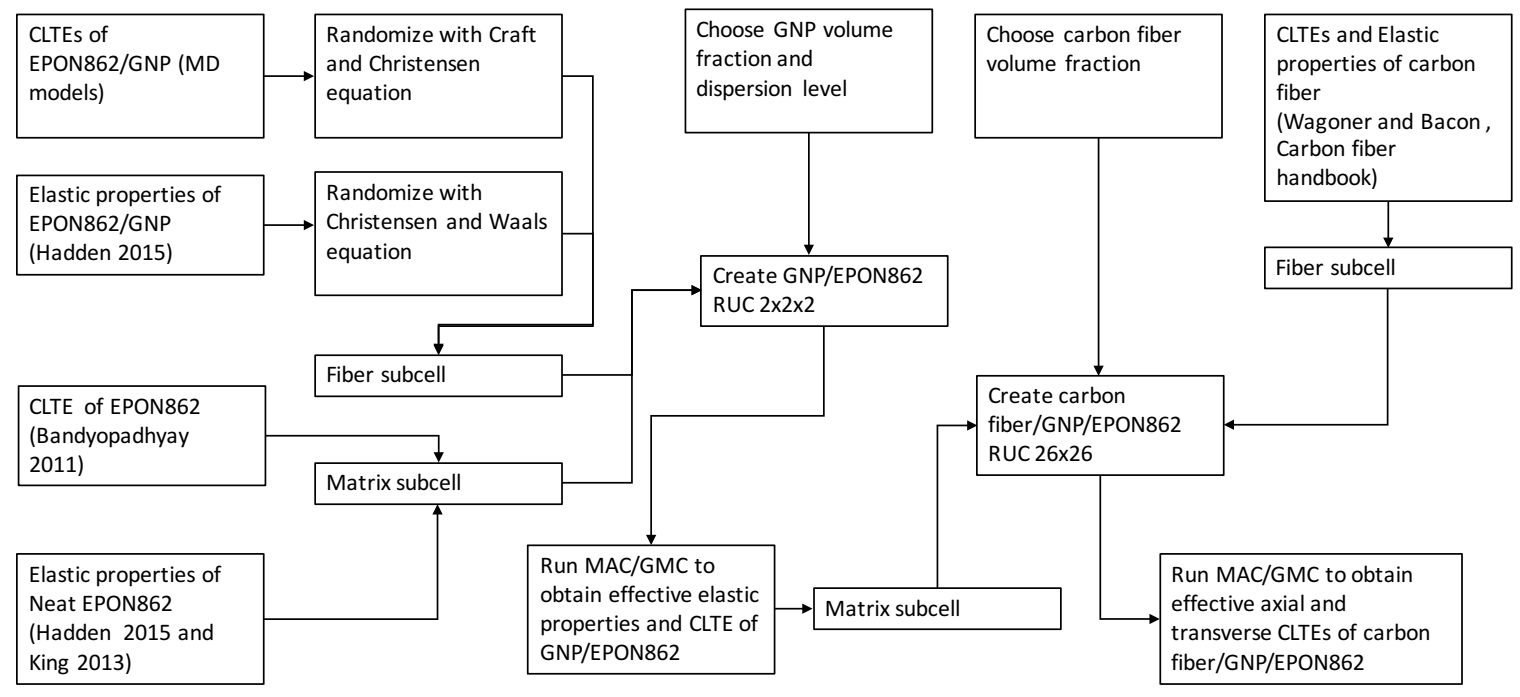

Schematic 2 The flow chart for calculating the effective coefficient of thermal expansion of GNP/CF/EPON862.

\subsubsection{MD modeling}

All four levels of dispersion GNP/EPON862 modeled by Hadden et al. [36] were used for this analysis. The $80 \%$ crosslinking models were selected as most realistic. The models 
were equilibrated by using NPT ensemble with the "aniso" type of barostat (as implemented in LAMMPS) at 1 atmosphere and 300K. The equilibration ran for $2 \mathrm{~ns}$ with 1 fs timesteps. The cut-off radius for airwise interactions was set at 10 angstroms. All equilibrated models were prepared by reducing the temperature to $173 \mathrm{~K}$ in the NPT ensemble with the aniso type of barostat set at 1 atmosphere. This preparation ran for 500 ps with 1 fs timesteps. Subsequently, simulations were performed with two different methods (heating-up and cooling-down) to determine the CLTE. The heating-up method continued from the preparation step by heating from $173 \mathrm{~K}$ up to $800 \mathrm{~K}$ using the NPT ensemble with aniso at 1 atmosphere for $5 \mathrm{~ns}$ with $1 \mathrm{fs}$ timesteps. The cooling-down method was continuously applied after the heating up method by decreasing the temperature from $800 \mathrm{~K}$ to $173 \mathrm{~K}$ with the same settings and simulation time. The temperatures and box lengths on each direction were individually averaged every 2 ps and exported to a log file. The CLTE was calculated using,

$$
\alpha=\frac{1}{\delta T} \frac{\delta L}{L}=\frac{1}{L} \frac{\delta L}{\delta T}
$$

where $\mathrm{L}$ is the length of box and $\mathrm{T}$ is the temperature of the system. The CLTEs of each system are shown in Table 15 on $\mathrm{x}, \mathrm{y}$, and $\mathrm{z}$ axes.

Table 15 Predicted CLTEs of GNP/EPON862 from MD simulations.

\begin{tabular}{ccccc}
\hline GNP layers & $\alpha_{x}\left(\mathrm{ppm} /{ }^{\circ} \mathrm{C}\right)$ & $\alpha_{y}\left(\mathrm{ppm} /{ }^{\circ} \mathrm{C}\right)$ & $\alpha_{z}\left(\mathrm{ppm} /{ }^{\circ} \mathrm{C}\right)$ & $\alpha_{\mathrm{r}}\left(\mathrm{ppm} /{ }^{\circ} \mathrm{C}\right)$ \\
\hline 1 & -7.343 & -9.344 & 260.015 & 9.955 \\
2 & -7.253 & -8.306 & 234.41 & 1.685 \\
3 & -7.943 & -6.482 & 210.944 & -1.526 \\
4 & -7.449 & -7.887 & 207.528 & -4.591 \\
\hline
\end{tabular}

\subsubsection{Micromechanics}

The CLTEs of GNP/epoxy models were randomized by using the equation by Craft et al. [108]. 


$$
\alpha_{r}=\frac{\left[E_{11}+4 v_{12}\left(1+v_{12}\right) K_{23}\right] \alpha_{1}+4\left(1+v_{12}\right) K_{23} \alpha_{2}}{E_{11}+4 v_{12}\left(1+v_{12}\right) K_{23}}
$$

The elastic properties in Equation (25) were taken from Table 14. The bulk modulus is defined as

$$
K_{23}=\frac{1}{2}\left[\frac{2}{E_{22}}-\frac{2 v_{12}^{2}}{E_{11}}-\frac{1}{2 G_{23}}\right]^{-1}
$$

where $G_{23}$ is transverse shear modulus which can be calculated by

$$
G_{23}=\frac{E_{22}}{2\left(1+v_{23}\right)}
$$

The randomized CLTE values are given in Table 15. The mechanical properties were also randomized by using Christensen \& Walls equation [109]

$$
\begin{gathered}
E_{r}=\frac{\left[E_{11}+\left(4 v_{12}^{2}+8 v_{12}+4\right) K_{23}\right]\left[E_{11}+\left(4 v_{12}^{2}-4 v_{12}+1\right) K_{23}+6\left(G_{12}+G_{23}\right)\right]}{3\left[2 E_{11}+\left(8 v_{12}^{2}+12 v_{12}+7\right) K_{23}+2\left(G_{12}+G_{23}\right)\right]} \\
v_{r}=\frac{E_{11}+\left(4 v_{12}^{2}+16 v_{12}+6\right) K_{23}-4\left(G_{12}+G_{23}\right)}{4 E_{11}+\left(16 v_{12}^{2}+24 v_{12}+14\right) K_{23}+4\left(G_{12}+G_{23}\right)}
\end{gathered}
$$

where $E_{11}, v_{1}$, and $G_{12}$ were the elastic properties given in Table $14, K_{23}$ and $G_{23}$ were calculated using Equation (26) and (27), respectively. The randomized elastic properties of GNP/EPON862 MD model are shown in Table 16.

Table 16 The randomized elastic moduli and Poisson's ratios of GNP/EPON862 MD models.

\begin{tabular}{ccc}
\hline GNP layers & $E_{r}(\mathrm{GPa})$ & $v_{r}$ \\
\hline 1 & 55.338 & 0.304 \\
2 & 93.247 & 0.246 \\
3 & 123.939 & 0.213 \\
4 & 149.264 & 0.194 \\
\hline
\end{tabular}


MAC/GMC [75] was used to determine the CLTE of the homogenized GNP/epoxy composite. A triply periodic 2x2x2 RUC (cubic packing array), shown in Figure 6 was employed, with the aspect ratio equal to 1 .

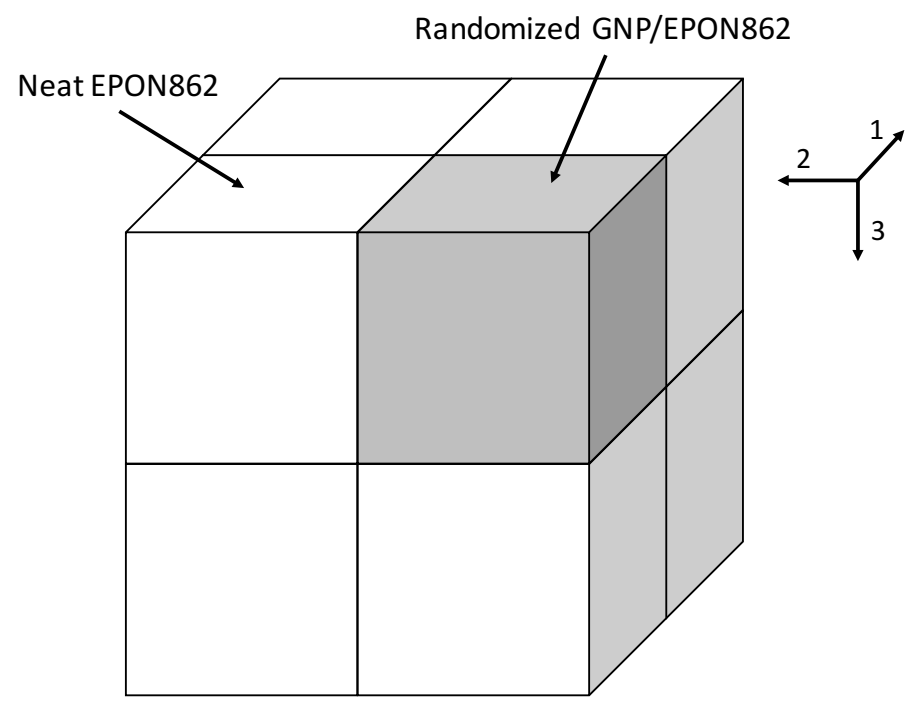

Figure 54 The representative simple cubic packing RUC.

The randomized CLTE of GNP/epoxy from Table 15 was applied as the inclusion region. The elastic properties of a system from Table 16 containing GNP/epoxy at completely random orientations were put into the same region. The elastic modulus $[\mathrm{E}=2.72 \mathrm{GPa}$ ] and properties Poisson's ratio $[v=0.43]$ of the pure epoxy are from Klimek-McDonald D [80] and Little et al. [110], respectively. The shear modulus was calculated by Equation (30) equal to $0.951 \mathrm{GPa}$ and the CLTE $\left[\alpha=86 \mathrm{ppm} /{ }^{\circ} \mathrm{C}\right]$ of the pure epoxy was taken from the work of Bandyopadhyay et al. [46].

$$
G=\frac{E}{2(1+v)}
$$

The volume fraction of the subcell containing the properties from the randomization of the MD results was calculated using Equation (31) to provide a consistent overall GNP volume fraction in the RUC. 


$$
v_{M D}^{R U C}=\frac{v_{G N P}^{R U C}}{v_{G N P}^{M D}}
$$

The total GNP volume fractions in RUC $\left(v_{G N P}^{R U C}\right)$ were specified from 0.01 to 0.10 with 0.005 of increments. The GNP volume fraction in MD was used from Table 14. The randomization of the GNP/epoxy occurred immediately after the MD calculations, and the randomized MD CLTEs were used in the micromechanics model of the GNP/Epoxy RUC. This workflow is analogous to that demonstrated by Hadden et al. [36] The error introduced when calculating the effective CLTE for the GNP/epoxy composite should be minimal because these properties are linear and mainly a function of the volume fraction of the constituents, and the overall orientation distribution of the inclusion.

The CLTEs of GNP/CF/EPON862 hybrid composite (lamina) model were calculated by using MAC/GMC as well. The $26 \times 26$ Circular Fiber Approximation, Rectangular Pack RUC (ARCHID=13) was employed to determine both axial and transverse CLTEs. The 312 middle subcells of this RUC are the fiber region which the materials properties of carbon fiber were added as shown in Table 17. The axial $\left(\alpha_{A}\right)$ and transverse $\left(\alpha_{T}\right)$ CLTEs of carbon fiber were from [111]. The volume fraction of carbon fiber was fixed at $56 \%$. The materials properties of randomized GNP/EPON862 were applied to the reset of subcells as the matrix region. All four different systems were studied in this hybrid composites. The volume fractions of GNP were also indicated in this region which were 0.01 to 0.10 with 0.005 increments.

Table 17 Elastic properties and CLTEs of carbon fiber.

\begin{tabular}{ccccccc}
\hline$E_{A}(\mathrm{GPa})$ & $E_{T}(\mathrm{GPa})$ & $G_{A}(\mathrm{GPa})$ & $v_{A}$ & $v_{T}$ & $\alpha_{A}\left(p p m /{ }^{\circ} \mathrm{C}\right)$ & $\alpha_{T}\left(p p m /{ }^{\circ} \mathrm{C}\right)$ \\
\hline 231 & 9.6 & 112 & 0.30 & 0.07 & -0.84 & 7.8 \\
\hline
\end{tabular}




\subsection{Results}

The calculated CLTEs are shown in Table 15. They were determined by averaging the results from the heating and cooling methods. The in-plane CLTEs ( $\mathrm{x}$ and $\mathrm{y}$ directions in Figure 5) were all negative values meaning that the in-plane dimension of box shrank at the higher temperature in those directions. Those contractions resulted the box expanded in z direction and the out-of-plane CLTE (z direction) was very high due to Poisson's contraction concept. The elastic properties used in Equation (25) were taken from Table 14, and the randomized CLTE values are given in Table 15.

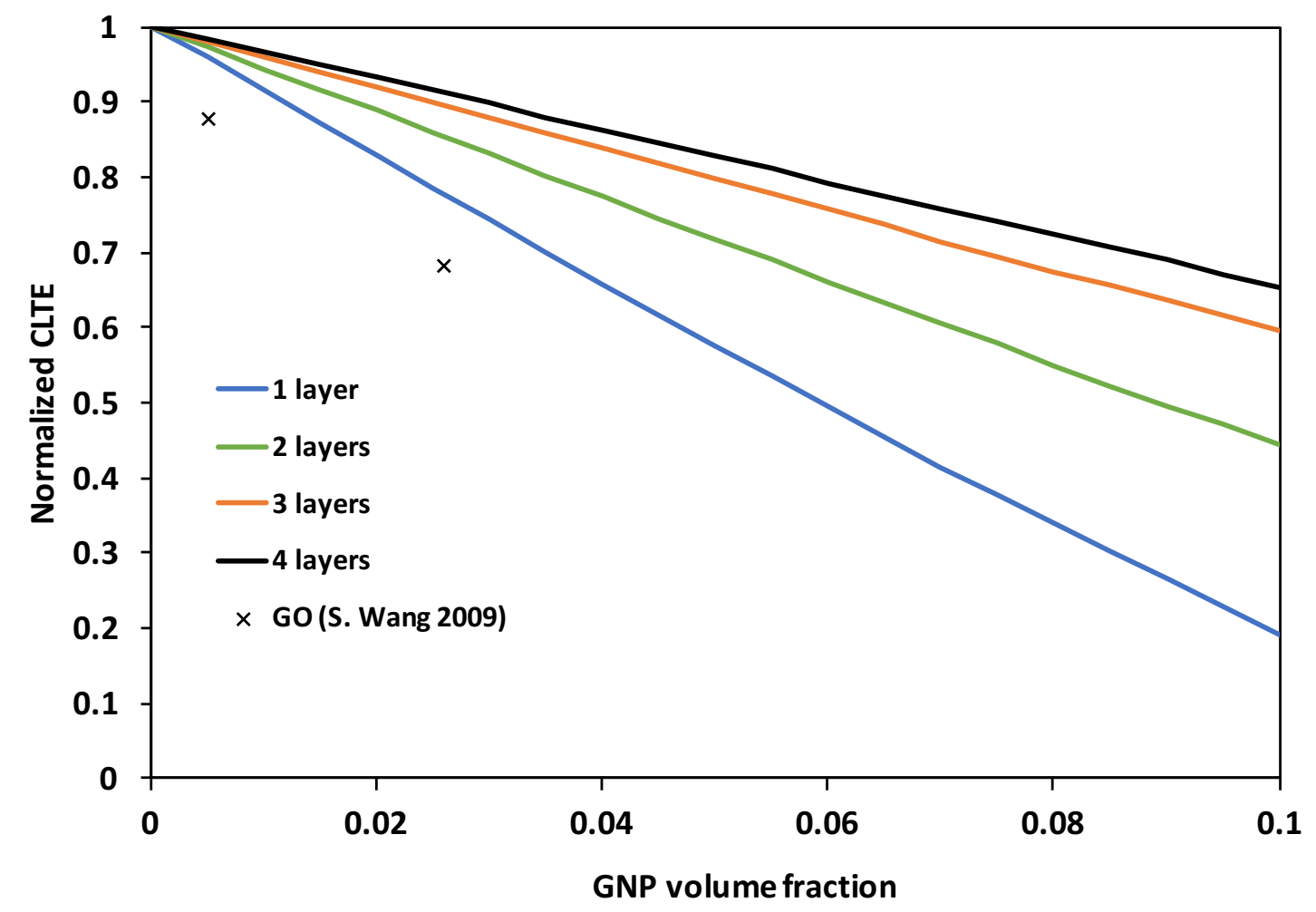

Figure 55 The plot of normalized CLTEs of GNP/EPON862 models as the function of GNP volume fraction compared with the experimental values [112].

Subsequent to randomization, the CLTE of the GNP/EPON862 system is calculated by homogenizing a $2 \times 2 \times 2$ RUC with MAC/GMC to account for the appropriate volume fraction of GNP. The volume fraction of the (inclusion) subcell which utilized the GNP/EPON862 CLTEs predicted using MD was calculated for overall GNP volume 
fractions ranging from $0 \%$ to $10 \%$ and different dispersion levels. The resulting normalized CLTEs which the ratio of CLTEs between GNP/EPON862 and neat EPON862 systems are shown in Figure 55. The plot shows that the different levels of GNP dispersion strongly affects to the CLTE. The perfectly dispersed GNP system is most decreased CLTE. The normalized CLTEs of graphene oxide (GO)/EPON862 specimens from experiment by Wang et al. [112] were also shown in Figure 55. The experimental value was approximately equivalent to the single GNP/EPON862 model. This is because the oxides on graphene facilitate the GNP exfoliation. We recognize that the GNP aspect ratio of this calculation was fix as the constant.

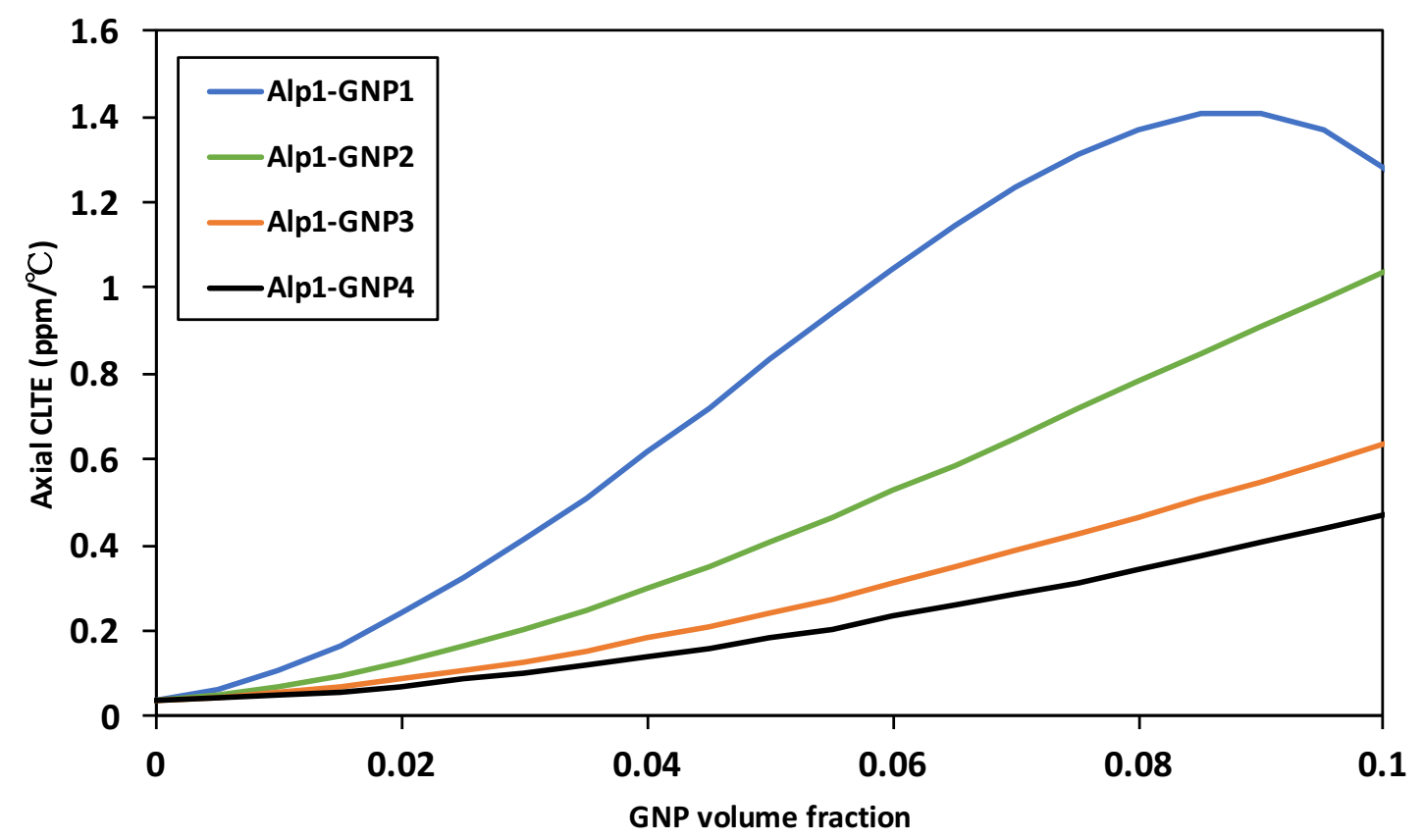

Figure 56 The plot of coefficient of thermal expansion along fiber direction as the function of GNP volume fraction.

Figure 56 and Figure 57 show the axial and transverse CLTEs of GNP/CF/EPON862 lamina calculated using MAC/GMC, respectively. The CLTE properties of matrix subcells were from the representative of homogenized GNP/EPON862 shown in Figure 55. The axial CLTEs of hybrid composites increase when increasing GNP volume fraction for all systems which the level of GNP dispersion influents the axial CLTEs. However, the axial CLTE of single-layer GNP system increases at the lower GNP volume fraction and then 
the axial CLTE decrease beyond 9 vol\% of GNP. The reason of this curve is not only about the changing of CLTE but also the changing of elastic properties in the matrix region. At the low GNP volume fraction, the axial CLTE increases because the stiffness in the matrix region increases and the axial CLTE of carbon fiber is very low. The CLTE of matrix slightly decreases which hardly affects to the overall axial CLTE of the hybrid composites. At the high GNP volume fraction, the CLTE of matrix dramatically decreases and consequentially overcomes the increasing CLTE due to the stiffness so the axial CLTE starts to decrease after this point.

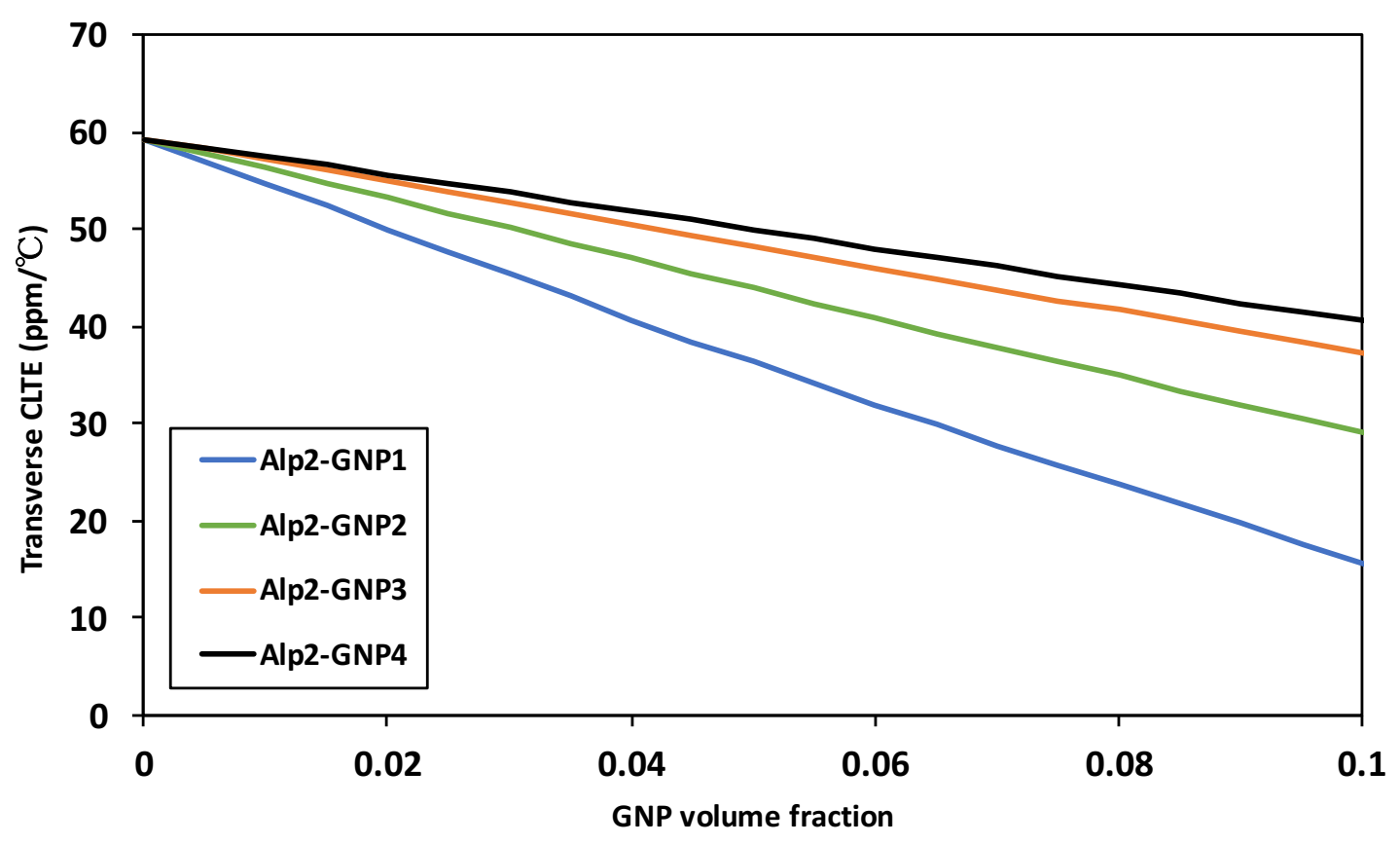

Figure 57 The plot of coefficient of transverse thermal expansion as the function of GNP volume fraction.

Figure 57 shows the transverse CLTEs of CF/GNP/EPON862 hybrid composites of all four different GNP systems. All transverse CLTEs lineally decrease when increasing the GNP volume fraction. The transverse CLTE of composite without GNP is already close to the CLTE of neat epoxy which is higher than the axial CLTE. Therefore, the effect of increasing stiffness does not appear on transverse CLTE. The plot also shows that the transverse CLTEs are depended on the different level of GNP dispersion which the perfect dispersion of GNP gives the most reduction of transverse CLTE of GNP/CF/EPON862 
hybrid composites. Moreover, the changing of transverse CLTE is larger an order of magnitude of the change of CLTE in axial direction.

\subsection{Conclusions}

For the GNP/EPON862 composites, GNP decreases the CLTE for all four different level of GNP dispersion. The perfectly dispersed GNP system has the most CLTE's reduction. The equation for randomizing CLTE by Craft \& Christensen and elastic properties by Christensen \& Walls can be used to predict the CLTE of GNP/CE system. Even the predicted CLTE of GNP/EPON862 deviates from the experimental value, the trend of CLTE reduction is in good agreement with experiment. For the GNP/CF/EPON862 hybrid composites, both axial and transverse CLTEs are depended on both GNP volume fraction and dispersion. The perfect dispersion of GNP has the most change in both CLTEs. The GNP inclusion in the hybrid composites strongly affects to the transverse CLTE. Finally, this study could be improve including GNP aspect ratio. 


\section{CHAPTER 7 IMPROVEMENT OF MICROMECHANICS CALCULATION INCLUDING GNP ASPECT RATIO}

\subsection{Introduction}

The improving of thermal conductivity in composites materials is extremely important as well as the enhancing mechanical properties. Graphene Nanoplatelet (GNP) in polymer composites has been widely investigated to develop the highly thermally conductive composites shown as Table 18. The characteristics of graphitic-based fillers; volume fraction, level of dispersion, orientation, and aspect ratio of fillers play roles to the effective thermal conductivity of composites. The experiments [29] have reported that the larger aspect ratio of GNP causes the relatively higher thermal conductivity in the specimen.

From Chapter 4 to Chapter 6, the GNP aspect ratio was all (Semi infinity) constant during the micromechanics calculation due to the original workflow from Hadden et al. [36]. This workflow the thermal conductivities of MD models which the GNP aspect ratios were infinity due to the periodic boundary condition were randomized by arithmetic average before putting into MAC/GMC. The resultant properties [36] of this method were shown in good agreement with the experiment for the elastic properties.

The micromechanics calculation of epoxy composites regarding aspect ratio of single wall carbon nanotube (CNT) has been studied by M. Radue [113]. There is the different perspective between 1D-infinity CNT and 2D-infinity GNP. In this chapter, we improve the workflow of micromechanics calculation for the thermal conductivity by taking into account the GNP aspect ratio. 
Table 18 The characteristics of graphitic-based filler in graphene/polymer composites studied thermal conductivity from literature.

\begin{tabular}{|c|c|c|c|c|c|c|c|c|c|c|}
\hline \multirow[b]{2}{*}{ Year } & \multirow[b]{2}{*}{ Author } & \multirow[b]{2}{*}{ Matrix } & \multicolumn{7}{|c|}{ Filler Characteristics } & \multirow[b]{2}{*}{ Ref } \\
\hline & & & Filler & $\begin{array}{l}\text { Lateral } \\
\text { length } \\
(\mathrm{nm})\end{array}$ & $\begin{array}{l}\text { Thickness } \\
(\mathrm{nm})\end{array}$ & $\begin{array}{l}\text { Aspect } \\
\text { ratio }\end{array}$ & $\begin{array}{l}\text { Number } \\
\text { layers }\end{array}$ & Amount & Method & \\
\hline 2006 & M.T. Hung et al. & $\begin{array}{l}\text { EPON862/ } \\
\text { DETDA }\end{array}$ & GNP & 3000 & 25 & 120 & $70 \mathrm{~b}$ & $1 \mathrm{vol} \%$ & SEM & {$[15]$} \\
\hline \multirow[t]{2}{*}{2007} & \multirow[t]{2}{*}{ A. Yu et al. } & \multirow[t]{2}{*}{$\begin{array}{l}\text { EPON862/ } \\
\text { DETDA }\end{array}$} & $\begin{array}{l}\text { GNP } \\
\text { GNP }\end{array}$ & 1700 & 1.7 & 200 & $169 \mathrm{~b}$ & $\begin{array}{l}1.3-25 \\
\text { vol } \% \\
2.1-5.4 \\
\text { vol } \%\end{array}$ & \multirow[t]{2}{*}{$\begin{array}{l}\text { AFM/ } \\
\text { TEM }\end{array}$} & \multirow[t]{2}{*}[29]{} \\
\hline & & & GNP & 1100 & 25 & 50 & $70 \mathrm{~b}$ & $5.4 \mathrm{vol} \%$ & & \\
\hline 2007 & B. Debelak et al. & $\begin{array}{l}\text { EPON862/ } \\
\text { DETDA }\end{array}$ & GNP & Unk. & Unk. & Unk. & Unk. & $20 \mathrm{wt} \%$ & - & {$[11]$} \\
\hline 2008 & S. Ganguli et al & $\begin{array}{l}\text { EPON862/ } \\
\text { DETDA }\end{array}$ & $\begin{array}{l}\text { Exfoliated } \\
\text { Graphite }\end{array}$ & 3900 & $2-100$ & $39-1950$ & $5-282 b$ & $2-20 w t \%$ & $\begin{array}{l}\text { Data } \\
\text { sheet }\end{array}$ & [14] \\
\hline 2009 & S. Wang, et la. & $\begin{array}{l}\text { EPON862/ } \\
\text { DETDA }\end{array}$ & GO & $2000^{*}$ & 1 & $2000^{*}$ & 1a & $1-5 \mathrm{wt} \%$ & AFM & [112] \\
\hline 2011 & C.C.Teng et al. & DGEBA/DDS & GNP & Unk. & 2.3 & Unk. & $6 \mathrm{~b}$ & $\begin{array}{l}0.24-3.85 \\
w t \%\end{array}$ & AFM & {$[25]$} \\
\hline 2011 & F. Yavari et al. & 1-octadecanol & graphene & Unk. & Unk. & Unk. & $3-4 a$ & $4 \mathrm{wt} \%$ & HRTEM & [114] \\
\hline 2011 & S. Yang et al. & DGEBA & MGNP & 4520 & 10 & 452 & $28 \mathrm{~b}$ & $1 \mathrm{wt} \%$ & $\begin{array}{l}\text { SEM/ } \\
\text { TEM }\end{array}$ & {$[28]$} \\
\hline 2012 & K.M.F. Shahil et al. & $\begin{array}{l}\text { EPON862/ } \\
\text { DETDA }\end{array}$ & MLG & $50-500$ & $0.71 b$ & $70-704$ & $2 a$ & $1.5-10 \mathrm{vol} \%$ & $\begin{array}{l}\text { AFM/ } \\
\text { SEM }\end{array}$ & {$[22]$} \\
\hline 2012 & S. Chatterjee et al. & $\begin{array}{l}\text { EPON828/Aroma } \\
\text { tic di-amine }\end{array}$ & $\begin{array}{l}\text { Expanded } \\
\text { GNP }\end{array}$ & Unk. & Unk. & Unk. & Unk. & $0.5-2 w t \%$ & - & {$[10]$} \\
\hline 2013 & C. Min et al. & $\begin{array}{l}\text { DGEBA/ } \\
\text { Anhydride }\end{array}$ & GNP & Unk. & $20-50$ & Unk. & $56-141 b$ & $\begin{array}{l}0.54-2.71 \\
\text { vol } \%\end{array}$ & SEM & [20] \\
\hline 2013 & $\begin{array}{l}\text { S. Chandrasekaran } \\
\text { et al. }\end{array}$ & $\begin{array}{l}\text { DGEBA/ } \\
\text { Anhydride }\end{array}$ & GNP & $\begin{array}{l}20000- \\
50000\end{array}$ & $12-15$ & $\begin{array}{l}1333- \\
4167\end{array}$ & $34-42 b$ & $1-2 \mathrm{wt} \%$ & $\begin{array}{l}\text { Data } \\
\text { sheet }\end{array}$ & [9] \\
\hline 2015 & J. W. Zha et al. & DGEBA & GNP & $\begin{array}{l}1000- \\
20000\end{array}$ & $5-15$ & $67-4000$ & $14-42 b$ & $2 \mathrm{vol} \%$ & $\begin{array}{l}\text { Data } \\
\text { sheet }\end{array}$ & [30] \\
\hline 2015 & $\begin{array}{l}\text { L. Diaz-Chacon et } \\
\text { al. }\end{array}$ & $\begin{array}{l}\text { DGEBA/ } \\
\text { Anhydride }\end{array}$ & GNP-m & 10000 & 45 & 200 & $126 \mathrm{~b}$ & $\begin{array}{l}0.26-2.67 \\
\text { vol } \%\end{array}$ & SEM & [19] \\
\hline \multirow{5}{*}{2015} & \multirow{5}{*}{ M. Shtein et al. } & \multirow{5}{*}{$\begin{array}{l}\text { DGEBA/ } \\
\text { Polyester } \\
\text { Triamine }\end{array}$} & GNP-M5 & 10000 & $5-20$ & 725 & $14-56 b$ & $\begin{array}{l}11.7-18.6 \\
\text { vol\% }\end{array}$ & \multirow{5}{*}{$\begin{array}{l}\text { SEM/TE } \\
\text { M/AFM }\end{array}$} & \multirow{5}{*}[23]{} \\
\hline & & & GNP-M15 & 19000 & $9-20$ & 1255 & $25-56 b$ & $\begin{array}{l}8.6-20.8 \\
\text { vol } \%\end{array}$ & & \\
\hline & & & GNP-M25 & 34000 & $>100$ & $<340$ & $>282 b$ & $\begin{array}{l}15.1-18.6 \\
\text { vol } \%\end{array}$ & & \\
\hline & & & GNP-C2 & 2000 & $5-20$ & 175 & $14-56 b$ & $\begin{array}{l}11.8-17.2 \\
\text { vol\% }\end{array}$ & & \\
\hline & & & GNP-H15 & 20000 & $15-100$ & 568 & $42-282 b$ & $\begin{array}{l}8.6-24.2 \\
\text { vol } \%\end{array}$ & & \\
\hline 2015 & Y. Wang et al. & CE/MHНРА & GNP & 10000 & 4 & 2500 & $10 \mathrm{a}$ & $0.5-8 w t \%$ & $\begin{array}{l}\text { SEM/TE } \\
\text { M/AFM }\end{array}$ & [27] \\
\hline \multirow[t]{2}{*}{2016} & $\begin{array}{l}\text { C. Kostagiannakopoulou } \\
\text { et al. }\end{array}$ & DGEBA & GNP & 5000 & $10-12$ & $417-500$ & $20-25$ & $1-15 w t \%$ & $\begin{array}{l}\text { Data } \\
\text { sheet }\end{array}$ & [17] \\
\hline & & CE/ACA & GNP & 1000 & $1.07-1.42 \mathrm{~b}$ & $704-939 b$ & $3-4$ & $4 \mathrm{wt} \%$ & $\begin{array}{l}\text { Data } \\
\text { sheet }\end{array}$ & Present \\
\hline
\end{tabular}

a - value from characterization

b - calculated value based on $0.355 \mathrm{~nm} /$ layer

* - estimated value from image 


\subsection{Micromechanics}

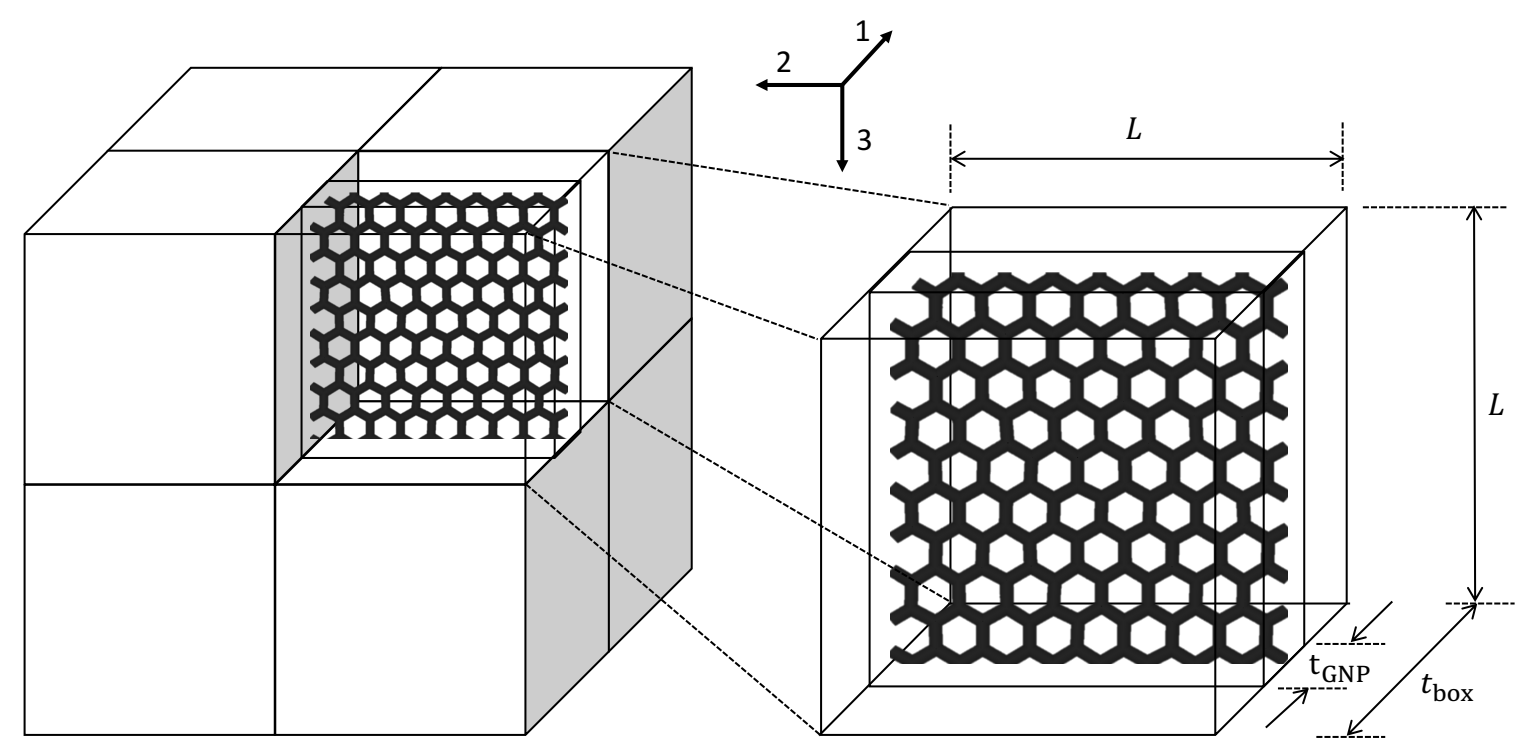

Figure 58 The dimensions of GNP and GNP/CE simulation box in RUC.

From Figure 58, the aspect ratio of GNP was defined as the proportion between the lateral length and the thickness of GNP. The total GNP aspect ratio of GNP/CE composites can be determined by

$$
a_{G N P}=\frac{L}{t_{G N P}}
$$

where $L$ is lateral length of GNP and $t_{G N P}$ is the GNP thickness. In MAC/GMC, the aspect ratio of fiber subcell $\left(a_{\text {cell }}\right)$ was defined as the ratio between box lengths as show following equation

$$
a_{c e l l}=\frac{t_{b o x}}{L}
$$


where $t_{b o x}$ and L are the box length along the out-of-plane and the in-plane direction of GNP, respectively. The equation (32) and (33) were combined and derived to obtain the relationship between $a_{c e l l}$ and $a_{G N P}$ shown as equation (34). The aspect ratio of subcell was determined after the GNP aspect ratio was specified.

$$
a_{c e l l}=\frac{t_{b o x}}{a_{G N P} \times t_{G N P}}
$$

From Figure 58, the doubly $2 \times 2 \times 2$ RUC was used to determine the thermal conductivity of GNP/CE composites with different amount of GNP. The in-plane thermal conductivity of GNP/CE was assigned both direction 2 and 3 of a GNP/CE subcell shown in Figure 58. The out-of-plane thermal conductivity was applied along direction 1. The thermal conductivity of neat CE was put in the other seven subcells. The total aspect ratios of the GNP $\left(a_{G N P}\right)$ were chosen to be 1, 10, 50, 100, 200, 1000, and 10000 which the subcell's aspect ratios $\left(a_{\text {cell }}\right)$ were calculated by using equation (34). The dimensions of GNP $\left(t_{G N P}\right)$ and MD simulation box $\left(t_{b o x}\right)$ are shown in Table 19. The thickness of GNP from MD was the result from OPLS-AA force field.

Table 19 The GNP thickness and simulation box length of each system.

\begin{tabular}{ccc}
\hline MD simulation systems & $\mathrm{t}_{\mathrm{GNP}}(\AA)$ & $\mathrm{t}_{\mathrm{box}}(\AA)$ \\
\hline $1 \mathrm{GNP} / \mathrm{CE}$ & 3.55 & 41.97 \\
$2 \mathrm{GNP} / \mathrm{CE}$ & 7.10 & 41.26 \\
$3 \mathrm{GNP} / \mathrm{CE}$ & 14.20 & 41.36 \\
$4 \mathrm{GNP} / \mathrm{CE}$ & 28.40 & 40.68 \\
\hline
\end{tabular}

The total GNP mass fractions were increased from 0 to $10 \mathrm{wt} \%$ with $1 \mathrm{wt} \%$ increments. The volume fraction of GNP/CE (fiber) subcell was calculated using equation (22). After the material properties were assigned to $\mathrm{MAC} / \mathrm{GMC}$, the thermal conductivities of GNP/CE were determined for all three axes. The arithmetic average of these three thermal conductivities was obtained to predict the thermal conductivity with all possible rotation 
of GNP. The GNP aspect ratio study was applied to all 1, 2, 3, and 4-layer GNP/CE systems.

Table 20 The characteristics of graphene fillers in experimental specimens $[22,29,112]$.

\begin{tabular}{cccccccccc}
\hline & & \multicolumn{9}{c}{ Filler Characteristic } & Amount & Ref \\
\cline { 3 - 7 } Matrix & Filler & $\begin{array}{c}\text { Lateral } \\
\text { length }(\mathrm{nm})\end{array}$ & $\begin{array}{c}\text { Thickness } \\
(\mathrm{nm})\end{array}$ & $\begin{array}{c}\text { Aspect } \\
\text { ratio }\end{array}$ & $\begin{array}{c}\text { Number } \\
\text { layers }\end{array}$ & Method & & \\
\hline $\begin{array}{c}\text { EPON862 } \\
\text { /DETDA }\end{array}$ & GNP & 350 & 1.7 & 200 & 4 & AFM, TEM & $\begin{array}{c}1.3-25 \\
\text { vol\% } \%\end{array}$ & {$[29]$} \\
$\begin{array}{c}\text { EPON862 } \\
\text { /DETDA }\end{array}$ & GO & $2000^{*}$ & 1 & $2000^{*}$ & 1 & AFM & $1-5 \mathrm{wt} \%$ & {$[112]$} \\
$\begin{array}{c}\text { EPON862 } \\
\text { /DETDA }\end{array}$ & MLG & $50-500$ & $0.71^{\mathrm{a}}$ & $70-704$ & 2 & AFM, SEM & $\begin{array}{c}1.5-10 \\
\text { vol\% } \%\end{array}$ & {$[22]$} \\
\hline
\end{tabular}

*estimated from AFM image

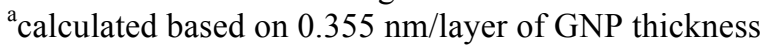

\subsection{Results}

The normalized thermal conductivities of 1-layer GNP/CE and 4-layer GNP/CE models with GNP aspect ratio of 1, 10, 50, 100, 200, 1000, and 10000 are shown in Figure 59 and Figure 60, respectively. The predicted thermal conductivities increase as nonlinear relationship with GNP mass fraction. Furthermore, the effective thermal conductivities are higher with the larger aspect ratio of GNP for both different level GNP dispersion. The thermal conductivities of 1-layer GNP/CE models are relatively higher than the thermal conductivities of 4-layer GNP/CE models at the same GNP aspect ratio. This means that the thermal conductivities are the higher with the better dispersion of GNP with same aspect ratio of GNP. 


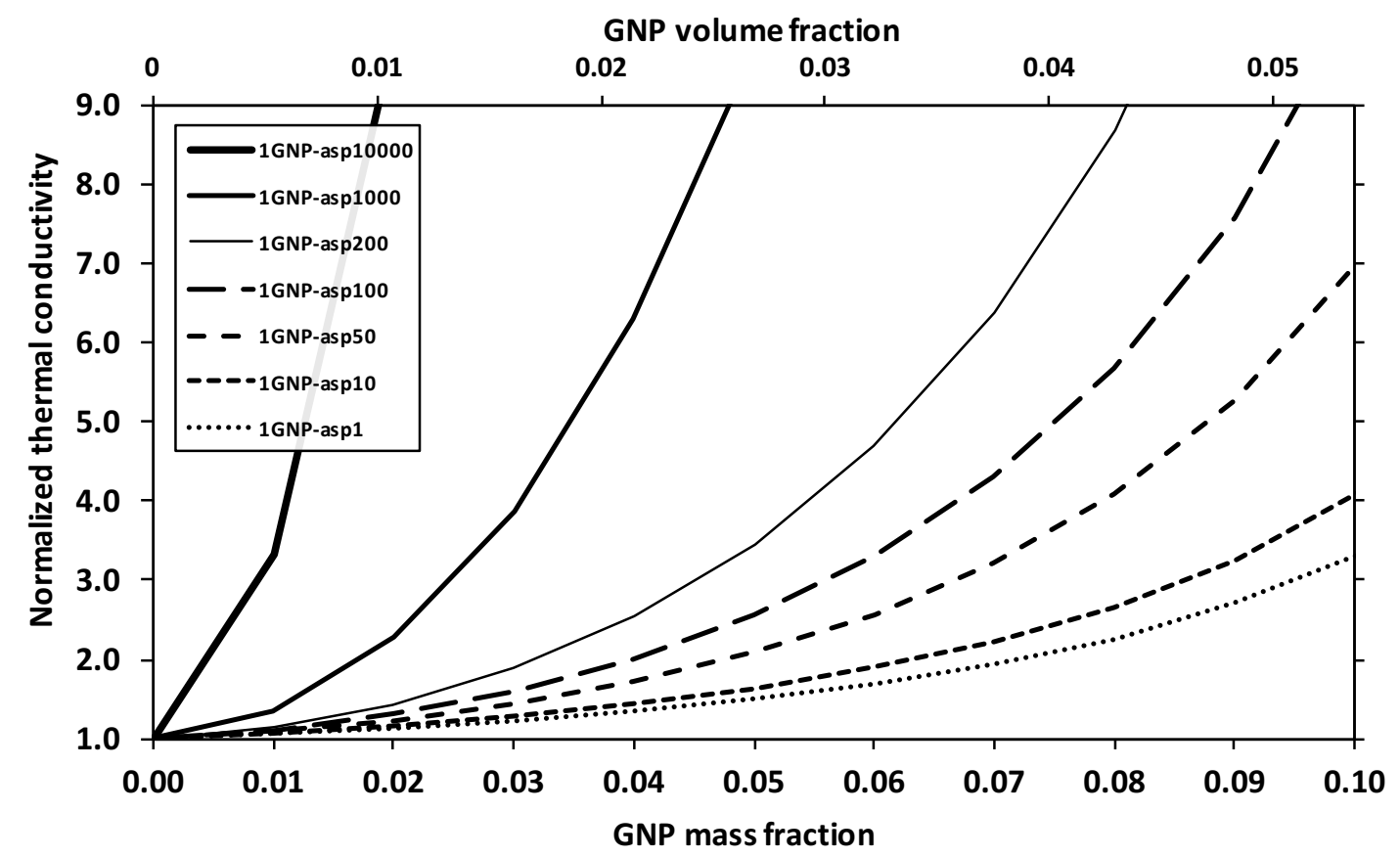

Figure 59 The predicted normalized thermal conductivity of single-layered GNP/CE model with different GNP aspect ratios as the function of GNP mass fraction.

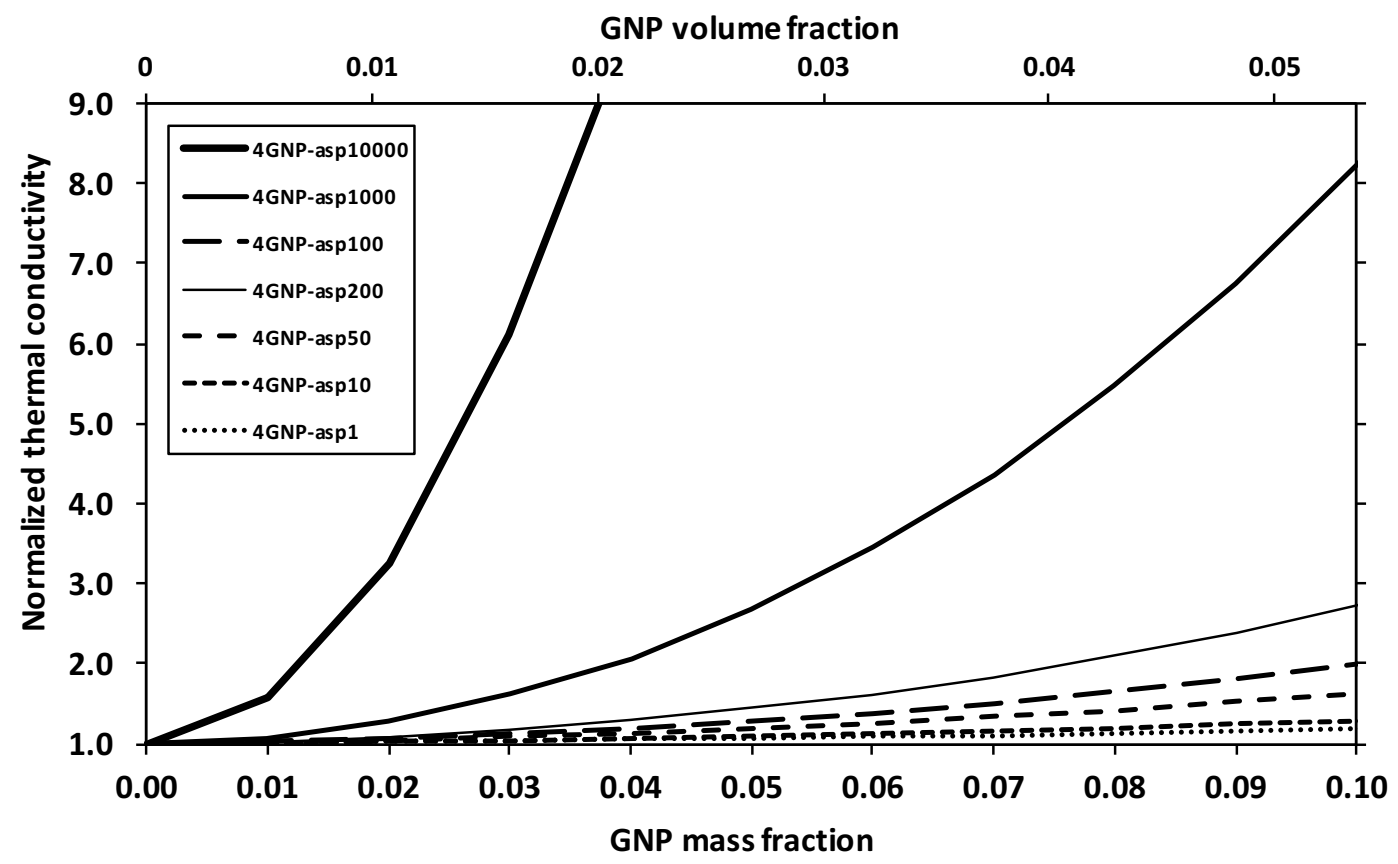

Figure 60 The predicted normalized thermal conductivity of 4-layered GNP/CE model with different GNP aspect ratios as the function of GNP mass fraction. 
Figure 61 shows the predicted normalized thermal conductivity of 2-layer GNP/CE model by using the multiscale modeling compared with the experimental normalized thermal conductivity from Shahil et al. [22]. The predicted thermal conductivities increase nonlinearly with the GNP volume fraction. The predicted thermal conductivity in agreement with the experimental thermal conductivity up to $5 \mathrm{vol} \%$ graphene. After $5 \mathrm{vol} \%$ of graphene, the thermal conductivities from the experiment are lower than the predicted values which possibly have the GNP agglomeration in the experimental specimens.

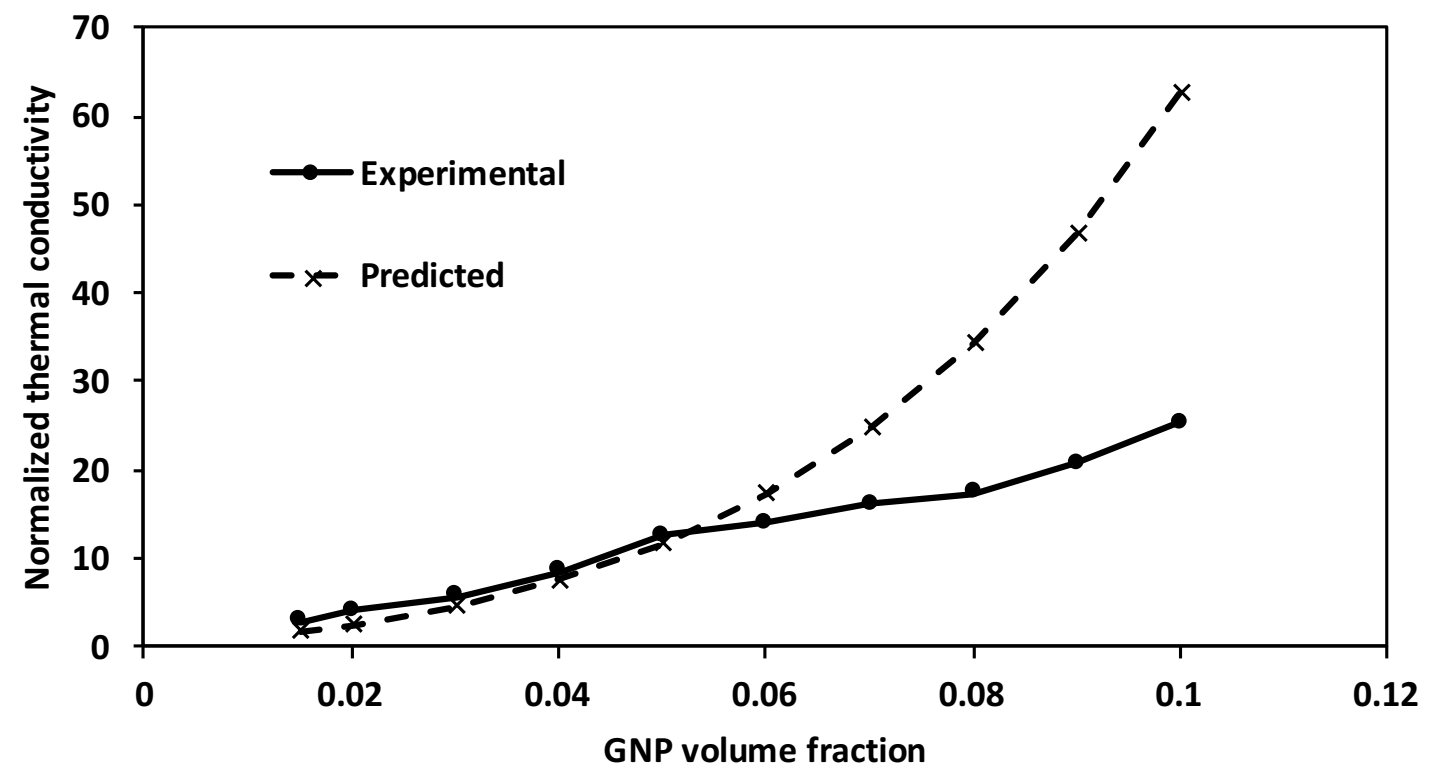

Figure 61 Plot of normalized thermal conductivities of 2-layer (Aspect ratio 704) GNP/CE models as the function of GNP volume fraction and the experimental values from Shahil et al. [22].

Figure 62 shows the normalized thermal conductivity for 4-layer GNP/CE model as the function of GNP volume fraction predicted by using multiscale modeling method. In this plot, the predicted values were determined by using 4-layer GNP system with 200 GNP aspect ratio compared with the experimental values from Yu et al. [29]. The predicted 
thermal conductivities increase nonlinearly with GNP volume fraction. At 10 vol\% GNP and below, the experimental normalized thermal conductivities are higher than the predicted results, however, they have similar trend. For the systems with 20 and 25 vol\% GNP, the normalized thermal conductivities for the experiment are lower than the predicted values which the GNP agglomeration possibly occurs in the experiment.

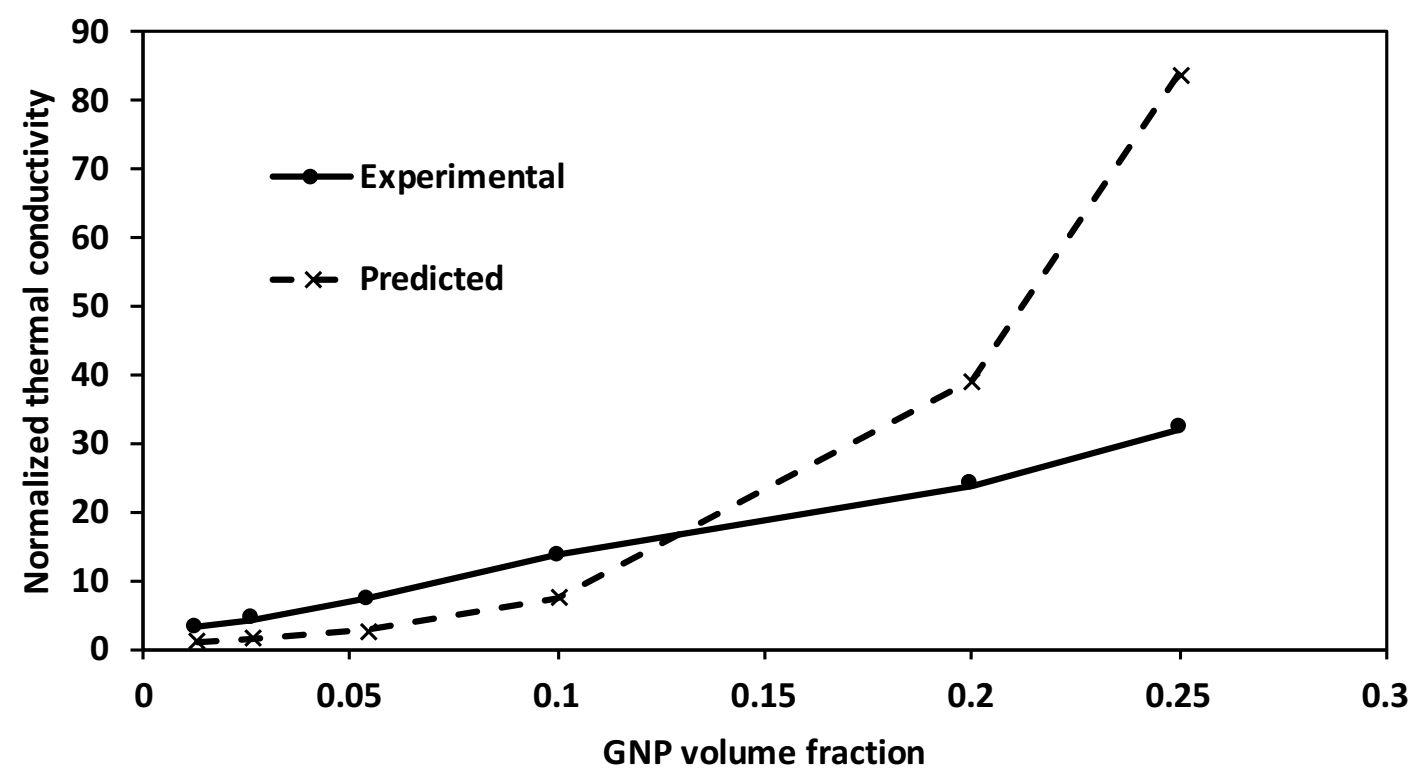

Figure 62 Plot of normalized thermal conductivities of 4-layer (Aspect ratio 200) GNP/CE models as the function of GNP volume fraction and the experimental values from $\mathrm{Yu}$ et al. [29].

Another prediction of thermal conductivity is shown in Figure 63. The predicted normalized thermal conductivities of GNP/CE models were calculated by specifying single-layer GNP with $2000 \mathrm{GNP}$ aspect ratio compared with experimental results taken from Wang et al. [112]. The predicted thermal conductivity increases linearly when having the higher GNP volume fraction. For 0.5 vol\% GNP, the predicted value is lower than the experiment. At $2.5 \mathrm{vol} \% \mathrm{GNP}$, the thermal conductivity from multiscale modeling is in good agreement with the experimental thermal conductivity. 


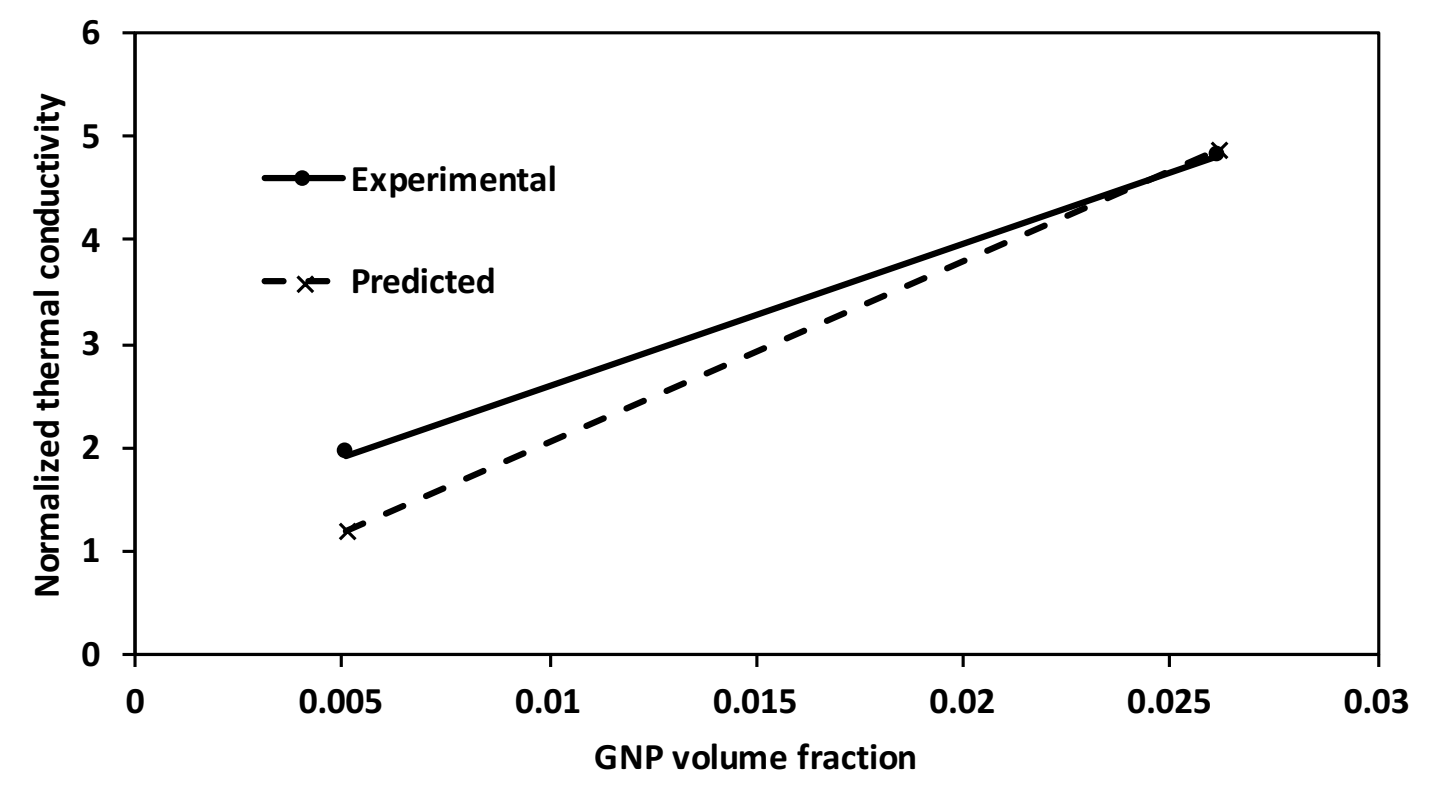

Figure 63 Plot of normalized thermal conductivities of 1-layer (Aspect ratio 2000) GNP/CE models as the function of GNP volume fraction and the experimental values from Wang et al. [112].

\subsection{Conclusions}

Micromechanical modeling can successfully predict the effective thermal conductivity of GNP/CE system by using MAC/GMC. GNP aspect ratio is one of important GNP characteristics to improve the thermal conductivity. The thermal conductivity does not only increase with the GNP volume fraction and better GNP dispersion but it also increases with the GNP aspect ratio. However, this study omitted the thermal pathway which the nanoparticles are connected and able to transfer the heat, effectively. This could further investigate the fully feature of GNP characteristics by using mesoscale modeling or finite element method. 


\section{CHAPTER 8 RECOMMENDATIONS FOR FUTURE WORK}

\subsection{Mesoscale Modeling}

Mesoscale modeling has capability to study the model at the larger scale combing with information from MD modeling. The thermal conductivity of GNP/CE is potentially investigated more about the orientation. This means how each different orientation GNP in a large model interacts to each other which the pathway or agglomeration can be captured at this level. The mixing of different level of GNP dispersion is another interesting topic to be studied because the experimental specimen always has the mix of different GNP thickness and lateral length.

\subsection{The Glass-Transition Temperature on GNP/CE Composites}

The glass-transition temperature of GNP/CE composites have not yet been studied which is an important materials property. The mechanical properties usually decrease when using the materials above the glass-transition temperature. The characteristic of GNP that could affect to the glass-transition temperature include the level of dispersion, aspect ratio, and orientation. 


\section{References}

1. Jorgensen, W.L., D.S. Maxwell, and J. TiradoRives, Development and testing of the OPLS all-atom force field on conformational energetics and properties of organic liquids. Journal of the American Chemical Society, 1996. 118(45): p. 11225-11236.

2. Robertson, M.J., J. Tirado-Rives, and W.L. Jorgensen, Improved Peptide and Protein Torsional Energetics with the OPLS-AA Force Field. Journal of Chemical Theory and Computation, 2015. 11(7): p. 3499-3509.

3. Jones, J.E., On the Determination of Molecular Fields. II. From the Equation of State of a Gas. Proceedings of the Royal Society of London. Series A, 1924. 106: p. 463-477.

4. Novoselov, K.S., et al., Electric field effect in atomically thin carbon films. Science, 2004. 306(5696): p. 666-669.

5. Balandin, A.A., Thermal properties of graphene and nanostructured carbon materials. Nature Materials, 2011. 10(8): p. 569-581.

6. Balandin, A.A., et al., Superior thermal conductivity of single-layer graphene. Nano Letters, 2008. 8(3): p. 902-907.

7. Lee, C., et al., Measurement of the elastic properties and intrinsic strength of monolayer graphene. Science, 2008. 321(5887): p. 385-388.

8. Cai, D.Y. and M. Song, Recent advance in functionalized graphene/polymer nanocomposites. Journal of Materials Chemistry, 2010. 20(37): p. 79067915.

9. Chandrasekaran, S., C. Seidel, and K. Schulte, Preparation and characterization of graphite nano-platelet (GNP)/epoxy nano-composite: Mechanical, electrical and thermal properties. European Polymer Journal, 2013. 49(12): p. 3878-3888.

10. Chatterjee, S., et al., Mechanical reinforcement and thermal conductivity in expanded graphene nanoplatelets reinforced epoxy composites. Chemical Physics Letters, 2012. 531: p. 6-10.

11. Debelak, B. and K. Lafdi, Use of exfoliated graphite filler to enhance polymer physical properties. Carbon, 2007. 45(9): p. 1727-1734.

12. Du, J.H. and H.M. Cheng, The Fabrication, Properties, and Uses of Graphene/Polymer Composites. Macromolecular Chemistry and Physics, 2012. 213(10-11): p. 1060-1077.

13. Fan, W., J.L. Li, and Y.Y. Zheng, Improved thermo-oxidative stability of three-dimensional and four-directional braided carbon fiber/epoxy hierarchical composites using graphene-reinforced gradient interface layer. Polymer Testing, 2015. 44: p. 177-185.

14. Ganguli, S., A.K. Roy, and D.P. Anderson, Improved thermal conductivity for chemically functionalized exfoliated graphite/epoxy composites. Carbon, 2008. 46(5): p. 806-817.

15. Hung, M.T., et al., Heat conduction in graphite-nanoplatelet-reinforced polymer nanocomposites. Applied Physics Letters, 2006. 89(2). 
16. Kandare, E., et al., Improving the through-thickness thermal and electrical conductivity of carbon fibre/epoxy laminates by exploiting synergy between graphene and silver nano-inclusions. Composites Part a-Applied Science and Manufacturing, 2015. 69: p. 72-82.

17. Kostagiannakopoulou, C., et al., Thermal Conductivity of Carbon Nanoreinforced Epoxy Composites. Journal of Nanomaterials, 2016.

18. Li, Y., et al., Graphite Nanoplatelet Modified Epoxy Resin for Carbon Fibre Reinforced Plastics with Enhanced Properties. Journal of Nanomaterials, 2017.

19. Lurayni Diaz-Chacon, R.M., Philippe Dieudonné, Jean Louis Bantignies, Said Tahir, Mehrdad Hassanzadeh, Eleida Sosa, Reinaldo Atencio, Graphite Nanoplatelets Composite Materials: Role of the Epoxy-System in the Thermal Conductivity. Journal of Materials Science and Chemical Engineering, 2015. 3(5): p. 75-87.

20. Min, C., et al., A graphite nanoplatelet/epoxy composite with high dielectric constant and high thermal conductivity. Carbon, 2013. 55: p. 116-125.

21. Potts, J.R., et al., Graphene-based polymer nanocomposites. Polymer, 2011. 52(1): p. 5-25.

22. Shahil, K.M.F. and A.A. Balandin, Graphene-Multilayer Graphene Nanocomposites as Highly Efficient Thermal Interface Materials. Nano Letters, 2012. 12(2): p. 861-867.

23. Shtein, M., et al., Thermally Conductive Graphene-Polymer Composites: Size, Percolation, and Synergy Effects. Chemistry of Materials, 2015. 27(6): p. 2100-2106.

24. Sun, X.B., et al., Dependence of the thermal conductivity of two-dimensional graphite nanoplatelet-based composites on the nanoparticle size distribution. Journal of Physics-Condensed Matter, 2010. 22(33).

25. Teng, C.C., et al., Thermal conductivity and structure of non-covalent functionalized graphene/epoxy composites. Carbon, 2011. 49(15): p. 51075116.

26. Tomasi, J.M., et al., Accelerated hydrothermal aging of cycloaliphatic epoxy/graphene nanoparticle composites. Polymer Degradation and Stability, 2016. 133: p. 131-135.

27. Wang, Y., et al., Enhanced Thermal and Electrical Properties of Epoxy Composites Reinforced With Graphene Nanoplatelets. Polymer Composites, 2015. 36(3): p. 556-565.

28. Yang, S.Y., et al., Synergetic effects of graphene platelets and carbon nanotubes on the mechanical and thermal properties of epoxy composites. Carbon, 2011. 49(3): p. 793-803.

29. Yu, A.P., et al., Graphite nanoplatelet-epoxy composite thermal interface materials. Journal of Physical Chemistry C, 2007. 111(21): p. 7565-7569.

30. Zha, J.W., et al., Tuning of thermal and dielectric properties for epoxy composites filled with electrospun alumina fibers and graphene 
nanoplatelets through hybridization. Journal of Materials Chemistry C, 2015. 3(27): p. 7195-7202.

31. Zhang, B., et al., Mechanical and Thermal Properties of Hierarchical Composites Enhanced by Pristine Graphene and Graphene Oxide Nanoinclusions. Journal of Applied Polymer Science, 2014. 131(19).

32. Zhou, T., et al., Improving the thermal conductivity of epoxy resin by the addition of a mixture of graphite nanoplatelets and silicon carbide microparticles. Express Polymer Letters, 2013. 7(7): p. 585-594.

33. King, J.A., et al., Mechanical properties of graphene nanoplatelet/epoxy composites. Journal of Composite Materials, 2015. 49(6): p. 659-668.

34. Klimek-McDonald, D.R., et al., Determination and Modeling of Mechanical Properties for Graphene Nanoplatelet/Epoxy Composites. Polymer Composites, 2016: p. n/a-n/a.

35. King, J.A., et al., Effects of carbon fillers on the conductivity and tensile properties of polyetheretherketone composites. Polymer Composites, 2016: p. n/a-n/a.

36. Hadden, C.M., et al., Mechanical properties of graphene nanoplatelet/carbon fiber/epoxy hybrid composites: Multiscale modeling and experiments. Carbon, 2015. 95: p. 100-112.

37. Hadden, C.M., et al., Molecular modeling of EPON-862/graphite composites: Interfacial characteristics for multiple crosslink densities. Composites Science and Technology, 2013. 76: p. 92-99.

38. Wang, T.Y. and J.L. Tsai, Investigating thermal conductivities of functionalized graphene and graphene/epoxy nanocomposites. Computational Materials Science, 2016. 122: p. 272-280.

39. Shen, X., et al., Effect of functionalization on thermal conductivities of graphene/epoxy composites. Carbon, 2016. 108: p. 412-422.

40. Shen, X., et al., Multilayer Graphene Enables Higher Efficiency in Improving Thermal Conductivities of Graphene/Epoxy Composites. Nano Letters, 2016. 16(6): p. 3585-3593.

41. Middleton, J., et al., The effect of ozone and high temperature on polymer degradation in polymer core composite conductors. Polymer Degradation and Stability, 2013. 98(11): p. 2282-2290.

42. Middleton, J., et al., The effect of ozone on polymer degradation in Polymer Core Composite Conductors. Polymer Degradation and Stability, 2013. 98(1): p. 436-445.

43. Fan, H.B. and M.M.F. Yuen, Material properties of the cross-linked epoxy resin compound predicted by molecular dynamics simulation. Polymer, 2007. 48(7): p. 2174-2178.

44. Varshney, V., et al., A molecular dynamics study of epoxy-based networks: Cross-linking procedure and prediction of molecular and material properties. Macromolecules, 2008. 41(18): p. 6837-6842.

45. Varshney, V., et al., Heat transport in epoxy networks: A molecular dynamics study. Polymer, 2009. 50(14): p. 3378-3385. 
46. Bandyopadhyay, A., et al., Molecular modeling of crosslinked epoxy polymers: The effect of crosslink density on thermomechanical properties. Polymer, 2011. 52(11): p. 2445-2452.

47. Li, C.Y. and A. Strachan, Molecular dynamics predictions of thermal and mechanical properties of thermoset polymer EPON862/DETDA. Polymer, 2011. 52(13): p. 2920-2928.

48. Bandyopadhyay, A. and G.M. Odegard, Molecular modeling of crosslink distribution in epoxy polymers. Modelling and Simulation in Materials Science and Engineering, 2012. 20(4).

49. Kravchenko, O.G., et al., Prediction of the chemical and thermal shrinkage in a thermoset polymer. Composites Part a-Applied Science and Manufacturing, 2014. 66: p. 35-43.

50. Odegard, G.M., et al., Predicting mechanical response of crosslinked epoxy using ReaxFF. Chemical Physics Letters, 2014. 591: p. 175-178.

51. Li, C.Y. and A. Strachan, Molecular Scale Simulations on Thermoset Polymers: A Review. Journal of Polymer Science Part B-Polymer Physics, 2015. 53(2): p. 103-122.

52. Radue, M.S., et al., Comparing the mechanical response of di-, tri-, and tetra-functional resin epoxies with reactive molecular dynamics. Journal of Polymer Science Part B: Polymer Physics: p. n/a-n/a.

53. Wu, C.F. and W.J. Xu, Atomistic molecular modelling of crosslinked epoxy resin. Polymer, 2006. 47(16): p. 6004-6009.

54. Fan, H.B., et al., Molecular dynamics simulation of thermal cycling test in electronic packaging. Journal of Electronic Packaging, 2007. 129(1): p. 3540.

55. Shenogina, N.B., et al., Molecular Modeling Approach to Prediction of Thermo-Mechanical Behavior of Thermoset Polymer Networks. Macromolecules, 2012. 45(12): p. 5307-5315.

56. Soni, N.J., P.H. Lin, and R. Khare, Effect of cross-linker length on the thermal and volumetric properties of cross-linked epoxy networks: A molecular simulation study. Polymer, 2012. 53(4): p. 1015-1019.

57. Kumar, A., V. Sundararaghavan, and A.R. Browning, Study of temperature dependence of thermal conductivity in cross-linked epoxies using molecular dynamics simulations with long range interactions. Modelling and Simulation in Materials Science and Engineering, 2014. 22(2).

58. Hamerton, I., et al., Towards the rational design of polymers using molecular simulation: Predicting the effect of cure schedule on thermomechanical properties for a cycloaliphatic amine-cured epoxy resin. Reactive \& Functional Polymers, 2014. 74: p. 1-15.

59. Komarov, P.V., et al., Highly cross-linked epoxy resins: an atomistic molecular dynamics simulation combined with a mapping/reverse mapping procedure. Macromolecules, 2007. 40(22): p. 8104-8113.

60. Stevens, G.C., Cure Kinetics of a Low Epoxide Hydroxyl-Group Ratio Bisphenol-a Epoxy-Resin Anhydride System by Infrared-Absorption 
Spectroscopy. Journal of Applied Polymer Science, 1981. 26(12): p. 42594278.

61. Park, S.J., et al., Cure and reaction kinetics of an anhydride-cured epoxy resin catalyzed by $N$-benzylpyrazinium salts using near-infrared spectroscopy. Polymer Engineering and Science, 2000. 40(12): p. 25692576.

62. Plimpton, S., Fast Parallel Algorithms for Short-Range MolecularDynamics. Journal of Computational Physics, 1995. 117(1): p. 1-19.

63. Jorgensen, W.L., OPLS All-Atom Parameters for Organic Molecules, lons, Peptides \& Nucleic Acids. 2008.

64. CambridgeSoft, ChemDraw. 2015, PerkinEImer, Inc.,.

65. Stukowski, A., Visualization and analysis of atomistic simulation data with OVITO-the Open Visualization Tool. Modelling and Simulation in Materials Science and Engineering, 2010. 18(1).

66. Zhang, M., Z.X. Huang, and Y. Qin, Quantum Chemical Calculation of Maleic Anhydride Ring-opening Reaction. Advanced Materials Research, 2009. 79: p. 1193-1196.

67. SC:1183-02 / EPIKOTE ${ }^{\mathrm{TM}}$ Resin 862/EPIKURE ${ }^{\mathrm{TM}}$ Curing Agent W System. Resolution Performance Products LLC.

68. Muggeo, V.M.R., Estimating regression models with unknown break-points. Statistics in Medicine, 2003. 22(19): p. 3055-3071.

69. Team, R., RStudio: Integrated Development. 2015, R. RStudio, Inc., Boston, MA

70. Kubo, R., M. Toda, and N. Hashitsume, Statistical Physics II Nonequilibrium Statistical Mechanics. 1991: Springer-Verlag Berlin Heidelberg.

71. Schelling, P.K., S.R. Phillpot, and P. Keblinski, Comparison of atomic-level simulation methods for computing thermal conductivity. Physical Review B, 2002. 65(14).

72. Vikas Varshney, A.K.R., Jeff Baur. Molecular Modeling of Thermo-Physical Properties of BMI Matrix and Its Interface with Amorphous Carbon Fiber. in Twenty-ninth Technical Conference on Composite Materials. 2014. Price Center, University of California San Diego, La Jolla, CA USA.

73. Jordan A. Whetsell, J.L., Mrinal C. Saha,M. Cengiz Altan, Effects of Sizing on Thermal Conductivity of Single Carbon Fibers in Longitudinal and Radial Directions, in 29th Annual American Society of Composite. September 2014: San Diego, CA.

74. Daniel, I.M. and O. Ishai, Engineering Mechanics of Composite Materials. 2 ed. 2006, Oxford University Press: New York.

75. Brett Bednarcyk and S.M. Arnold, MAC/GMC user's manual - keywords manual. Vol. 2. 2002.

76. Tomasi, J.M., J.A. King, A.S. Krieg, I. Miskioglu, G.M. Odegard, Thermal, electrical, and mechanical properties of talc- and glass microsphereReinforced Cycloaliphatic epoxy composites. Polymer Composites, 2017. 
77. International, A., ASTM D792-13, Standard Test Methods for Density and Specific Gravity (Relative Density) of Plastics by Displacement. 2013: West Conshohocken, PA.

78. International, A., ASTM D638-14, Standard Test Method for Tensile Properties of Plastics. 2014: West Conshohocken, PA.

79. International, A., ASTM F433-02(2014)e1, Standard Practice for Evaluating Thermal Conductivity of Gasket Materials. 2014: West Conshohocken, PA.

80. Klimek-McDonald, D.R., MECHANICAL PROPERTIES OF GRAPHENE NANOPLATELET/EPOXY COMPOSITES, in Department of Chemical Engineering. 2015, Michigan Technological University.

81. Ferry, J.D., Viscoelastic properties of polymers. 1980, New York: John Wiley \& Sons Inc.

82. Chen, Z., et al., Effect of Chain Flexibility of Epoxy Encapsulants on the Performance and Reliability of Light-Emitting Diodes. Industrial \& Engineering Chemistry Research, 2016. 55(28): p. 7635-7645.

83. Zhao, L.N., L. Zhang, and Z.G. Wang, Synthesis and degradable properties of cycloaliphatic epoxy resin from renewable biomass-based furfural. Rsc Advances, 2015. 5(115): p. 95126-95132.

84. Liu, W.S., et al., Synthesis and properties of two novel silicon-containing cycloaliphatic epoxy resins for electronic packaging application. Polymers for Advanced Technologies, 2012. 23(3): p. 367-374.

85. Xie, M.R. and Z.G. Wang, Synthesis and properties of a novel cycloaliphatic epoxide. Macromolecular Rapid Communications, 2001. 22(8): p. 620-623.

86. Wong, C.P. and R.S. Bollampally, Thermal conductivity, elastic modulus, and coefficient of thermal expansion of polymer composites filled with ceramic particles for electronic packaging. Journal of Applied Polymer Science, 1999. 74(14): p. 3396-3403.

87. Fang, L.J., et al., Nano-micro structure of functionalized boron nitride and aluminum oxide for epoxy composites with enhanced thermal conductivity and breakdown strength. Rsc Advances, 2014. 4(40): p. 21010-21017.

88. Gao, J., et al., Enhanced Thermal Properties for Epoxy Composites with a Three-dimensional Graphene Oxide Filler. Fibers and Polymers, 2015. 16(12): p. 2617-2626.

89. Peng, W.Y., et al., Electrical and thermophysical properties of epoxy/aluminum nitride nanocomposites: Effects of nanoparticle surface modification. Composites Part a-Applied Science and Manufacturing, 2010. 41(9): p. 1201-1209.

90. Yan, H.Y., et al., Thermal Conductivity of Magnetically Aligned GraphenePolymer Composites with Fe3O4-Decorated Graphene Nanosheets. Journal of Electronic Materials, 2015. 44(2): p. 658-666.

91. $\mathrm{Yu}, \mathrm{J} . \mathrm{H}$. , et al., Influence of nano-AIN particles on thermal conductivity, thermal stability and cure behavior of cycloaliphatic epoxy/trimethacrylate system. Express Polymer Letters, 2011. 5(2): p. 132-141. 
92. $\mathrm{Yu}, \mathrm{J} . \mathrm{H}$. , et al., Interfacial modification of boron nitride nanoplatelets for epoxy composites with improved thermal properties. Polymer, 2012. 53(2): p. 471-480.

93. Zhong, $\mathrm{H}$. and S. Rubinsztajn, Organic matrices containing nanomaterials to enhance bulk thermal conductivity. 2006, Google Patents.

94. Villiere, M., et al., Experimental determination and modeling of thermal conductivity tensor of carbon/epoxy composite. Composites Part a-Applied Science and Manufacturing, 2013. 46: p. 60-68.

95. Han, S. and D.D.L. Chung, Increasing the through-thickness thermal conductivity of carbon fiber polymer-matrix composite by curing pressure increase and filler incorporation. Composites Science and Technology, 2011. 71(16): p. 1944-1952.

96. Liang, J., M.C. Saha, and M.C. Altan, Effect of Carbon Nanofibers on Thermal Conductivity of Carbon Fiber Reinforced Composites. Procedia Engineering, 2013. 56(Supplement C): p. 814-820.

97. Wang, S., et al., Heterogeneously structured conductive carbon fiber composites by using multi-scale silver particles. Composites Part B: Engineering, 2014. 61(Supplement C): p. 172-180.

98. Alawar, A., E.J. Bosze, and S.R. Nutt, A composite core conductor for low sag at high temperatures. leee Transactions on Power Delivery, 2005. 20(3): p. 2193-2199.

99. Pop, E., V. Varshney, and A.K. Roy, Thermal properties of graphene: Fundamentals and applications. Mrs Bulletin, 2012. 37(12): p. 1273-1281.

100. S. Chinkanjanarot, et al. Predicting Thermal Conductivity of Graphene Nanoplatelet/Epoxy Nanocomposite using Non-Equilibrium Molecular Dynamics. in American Society for Composites 31st Technical Conference. 2016. Williamsburg, VA.

101. Dodd Gray, A.M., Bhaskar Mookerji, Crystal Structure of Graphite, Graphene and Silicon. Physics for Solid State Applications, 2009.

102. Fu, S.Y. and Y.W. Mai, Thermal conductivity of misaligned short-fiberreinforced polymer composites. Journal of Applied Polymer Science, 2003. 88(6): p. 1497-1505.

103. Nicholas A. Fasanella, V.S., Thermal conductivity via atomistic modeling for epoxy-SWNT composites, in 57th AIAA/ASCE/AHS/ASC Structures, Structural Dynamics, and Materials Conference. 2016, AIAA SciTech: San Diego, California, USA.

104. Kim, H., A.A. Abdala, and C.W. Macosko, Graphene/Polymer Nanocomposites. Macromolecules, 2010. 43(16): p. 6515-6530.

105. Wang, Y., et al., Effect of Covalent Functionalization on Thermal Transport across Graphene-Polymer Interfaces. Journal of Physical Chemistry C, 2015. 119(22): p. 12731-12738.

106. Sun, X.B., et al., Oxidized Graphite Nanoplatelets as an Improved Filler for Thermally Conducting Epoxy-Matrix Composites. Journal of Electronic Packaging, 2011. 133(2). 
107. Zhu, J., et al., Improving the dispersion and integration of single-walled carbon nanotubes in epoxy composites through functionalization. Nano Letters, 2003. 3(8): p. 1107-1113.

108. Craft, W.J. and R.M. Christensen, Coefficient of Thermal-Expansion for Composites with Randomly Oriented Fibers. Journal of Composite Materials, 1981. 15(Jan): p. 2-20.

109. Christensen, R.M. and F.M. Waals, Effective Stiffness of Randomly Oriented Fibre Composites. Journal of Composite Materials, 1972. 6(3): p. 518-535.

110. Littell, J.D., et al., Measurement of epoxy resin tension, compression, and shear stress-strain curves over a wide range of strain rates using small test specimens. Journal of Aerospace Engineering, 2008. 21(3): p. 162-173.

111. Wagoner G. and B. R., Extended Abstract, in 19th Biennial Conf. on Carbon. 1989: State Cooledge, Pennsylvania. p. 296-297.

112. Wang, S.R., et al., Thermal Expansion of Graphene Composites. Macromolecules, 2009. 42(14): p. 5251-5255.

113. Radue, M.S., MOLECULAR MODELING OF AEROSPACE POLYMER MATRICES INCLUDING CARBON NANOTUBE-ENHANCED EPOXY, in Mechanical Engineering - Engineering Mechanics. 2017, MICHIGAN TECHNOLOGICAL UNIVERSITY: MICHIGAN TECHNOLOGICAL UNIVERSITY.

114. Yavari, F., et al., Enhanced Thermal Conductivity in a Nanostructured Phase Change Composite due to Low Concentration Graphene Additives. Journal of Physical Chemistry C, 2011. 115(17): p. 8753-8758. 


\section{Appendix A Computational Resources}

\section{A.1 HPC specification (Michigan Tech)}

\begin{tabular}{lll}
\hline & Athena & Superior \\
\hline No. of compute nodes & 27 & 72 \\
No. of CPU cores per node & 12 & 16 \\
CPU type & Intel Xeon X5650 & Intel Sandy Bridge E5-2670 \\
CPU speed & $2.67 \mathrm{GHz}$ & $2.60 \mathrm{GHz}$ \\
RAM per node & $24 \mathrm{~GB}$ & $64 \mathrm{~GB}$ \\
Storage & $13 \mathrm{~TB}$ & $48 \mathrm{~TB}$ \\
\hline
\end{tabular}

\section{A.2 Computing Time}

Most of the MD simulations were run on Superior HPC, few of monomers simulation were run on Athena HPC.

\section{A.2.1 Neat Cycloaliphatic Epoxy}

Number of atoms per model: 7164 (Cube) and 10746 (Prism)

\begin{tabular}{|c|c|c|c|c|}
\hline System & Simulation Description & $\begin{array}{l}\text { Simulation Time } \\
\text { (ns) }\end{array}$ & $\begin{array}{l}\text { No. of } \\
\text { Proc. }\end{array}$ & $\begin{array}{l}\text { CPU time } \\
(\mathrm{hrs})\end{array}$ \\
\hline & Monomers* & 3 & $16-32$ & 3 \\
\hline \multirow{4}{*}{$\begin{array}{l}\text { Cube - } \\
\text { mechincal } \\
\text { properties }\end{array}$} & Densification & 26.5 & 16 & 591 \\
\hline & Crosslink - 100 cycles & 2 & 64 & 5567 \\
\hline & Relaxation & 10.5 & 16 & 652 \\
\hline & Tensile deformation & 30 & 16 & 4414 \\
\hline \multirow{4}{*}{$\begin{array}{l}\text { Prism - } \\
\text { thermal } \\
\text { properties }\end{array}$} & Densification & 26.5 & 16 & 1435 \\
\hline & Crosslink - reaching to $90 \%$ & 15.5 & 16 & 3185 \\
\hline & Relaxation & 73.5 & 16 & 2992 \\
\hline & NEMD for thermal conductivity & 70.35 & 16 & 4791 \\
\hline \multirow{4}{*}{$\begin{array}{l}\text { Cube - } \\
\text { thermal } \\
\text { properties }\end{array}$} & Crosslink - reaching to $90 \%$ & 11.42 & 16 & 1069 \\
\hline & Relaxation & 73.5 & 16 & 1780 \\
\hline & $\begin{array}{l}\text { Heating-up simulation for } \mathrm{Tg} \text { and } \\
\text { CLTE }\end{array}$ & 192.5 & 16 & 8712 \\
\hline & EMD for thermal conductivity & 350.35 & 16 & 13179 \\
\hline
\end{tabular}

*Using Athena and Superior HPC 


\section{A.2.2 Graphene Nanoplatelet/Cycloaliphatic Epoxy}

Number of atoms per model: 5886-5949

\begin{tabular}{llll}
\hline Simulation Description & Simulation Time (ns) & No. of Proc. & CPU time (hrs) \\
\hline Graphene & 0.8 & 16 & 4 \\
Densification & 106 & 16 & 5346 \\
Crosslink - reaching to 80\% & 55.7 & 16 & 4691 \\
Relaxation & 42 & 16 & 1192 \\
EMD for thermal conductivity & 200.2 & 16 & 7320 \\
\hline
\end{tabular}

\section{A.2.3 Functionalized Graphene Nanoplatelet/Cycloaliphatic Epoxy}

Number of atoms per model: 5989-6029

\begin{tabular}{llll}
\hline Simulation Description & Simulation Time (ns) & No. of Proc. & CPU time (hrs) \\
\hline Functionalized graphene & 3.2 & 16 & 19 \\
Densification & 53 & 16 & 1991 \\
Crosslink - reaching to $80 \%$ & 31.12 & 16 & 14278 \\
Relaxation & 21 & 16 & 1430 \\
EMD for thermal conductivity & 100.1 & 16 & 3684 \\
\hline
\end{tabular}

\section{A.2.4 CLTE of GNP/EPON862}

Number of atoms per model: 24950-37550

\begin{tabular}{llll}
\hline Simulation Description & Simulation Time (ns) & No. of Proc. & CPU time (hrs) \\
\hline Relaxation & 8.4 & 16 & 816 \\
Heating up and cooling down & 42 & 16 & 3494 \\
\hline
\end{tabular}




\section{Appendix B Copyright documentation}

B.1 Copyright of Figure 1, Figure 2, and Figure 3

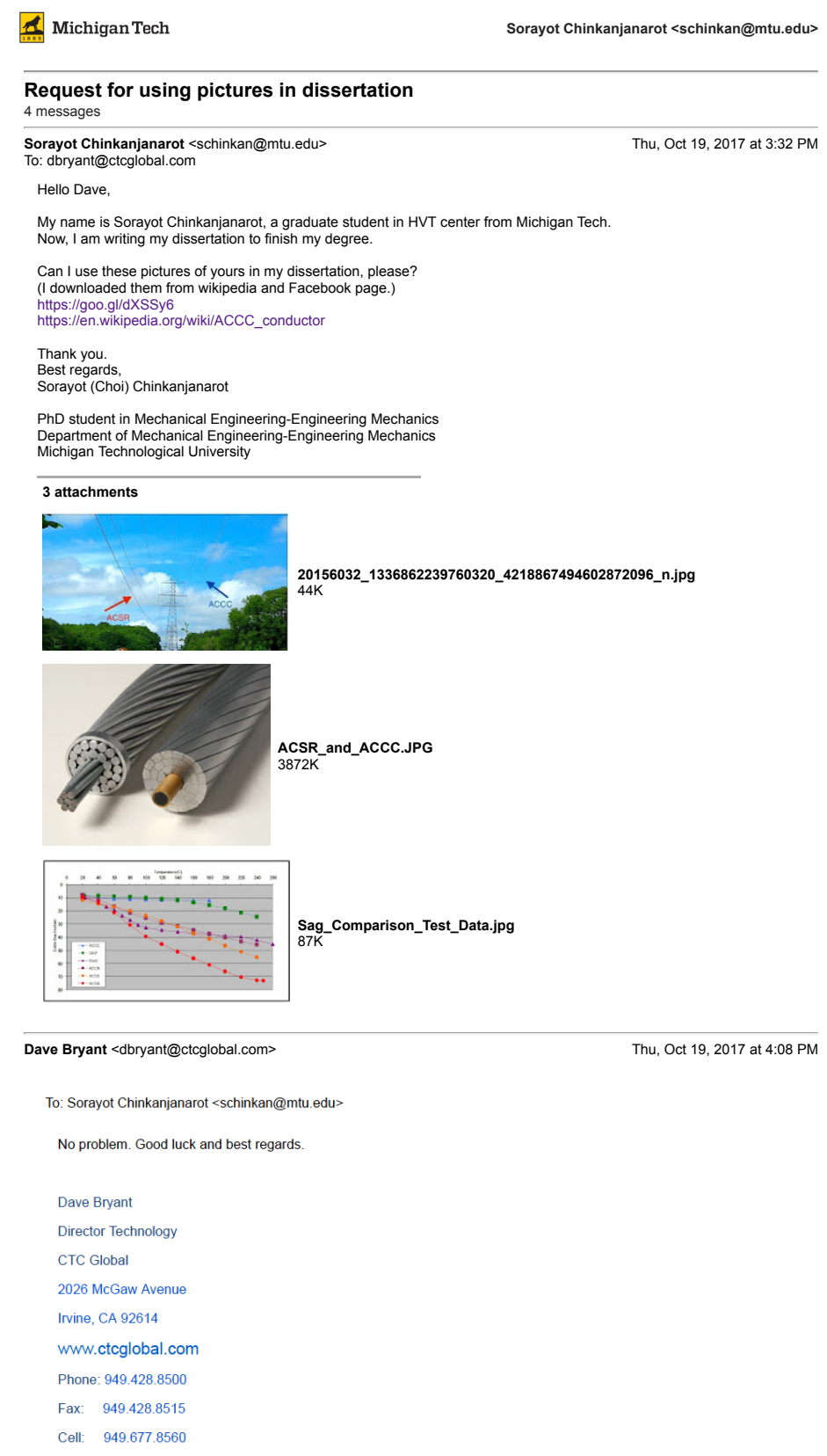




\section{B.2 Copyright of Figure 6}

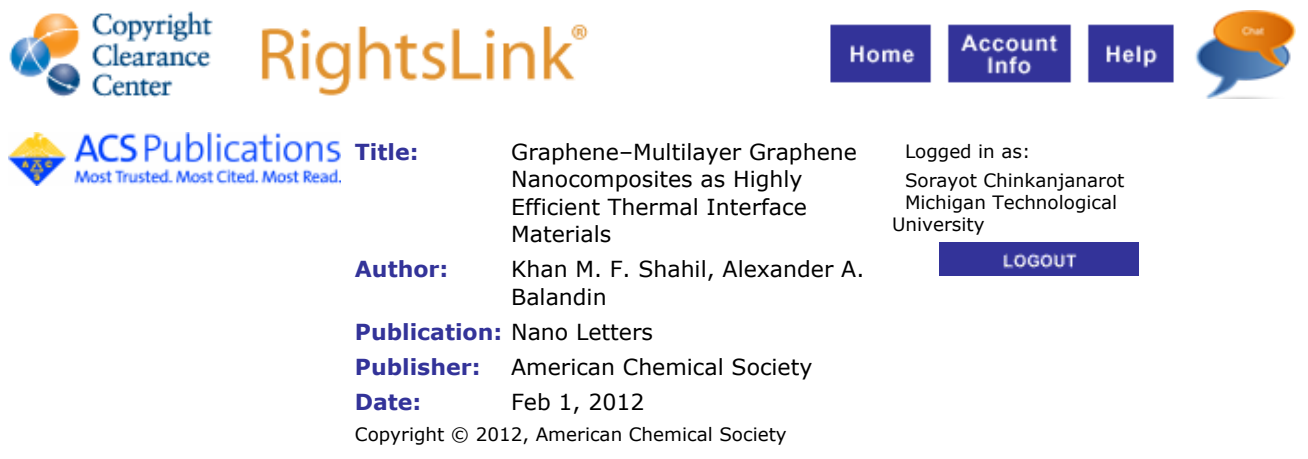

\section{PERMISSION/LICENSE IS GRANTED FOR YOUR ORDER AT NO CHARGE}

This type of permission/license, instead of the standard Terms \& Conditions, is sent to you because no fee is being charged for your order. Please note the following:

- Permission is granted for your request in both print and electronic formats, and translations.

- If figures and/or tables were requested, they may be adapted or used in part.

- Please print this page for your records and send a copy of it to your publisher/graduate school.

- Appropriate credit for the requested material should be given as follows: "Reprinted (adapted) with permission from (COMPLETE REFERENCE CITATION). Copyright (YEAR) American Chemical Society." Insert appropriate information in place of the capitalized words.

- One-time permission is granted only for the use specified in your request. No additional uses are granted (such as derivative works or other editions). For any other uses, please submit a new request.

If credit is given to another source for the material you requested, permission must be obtained from that source.

\section{BACK CLOSE WINDOW}




\section{Appendix C Codes and Scripts}

\section{C.1 Densification script}

This script is to compress the simulation box to general mass density of epoxy as mentioned above.

\#----------initialization----------

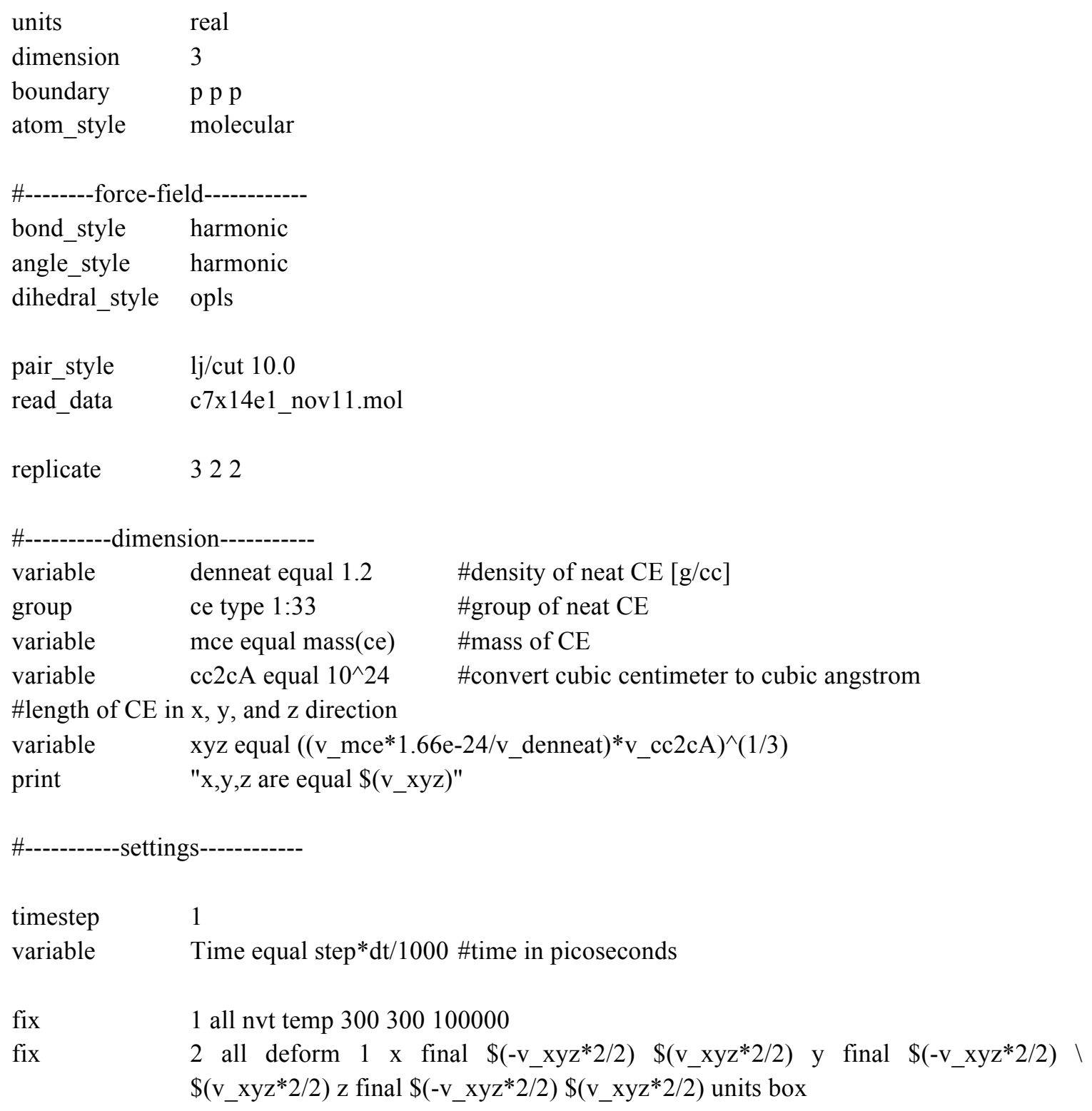




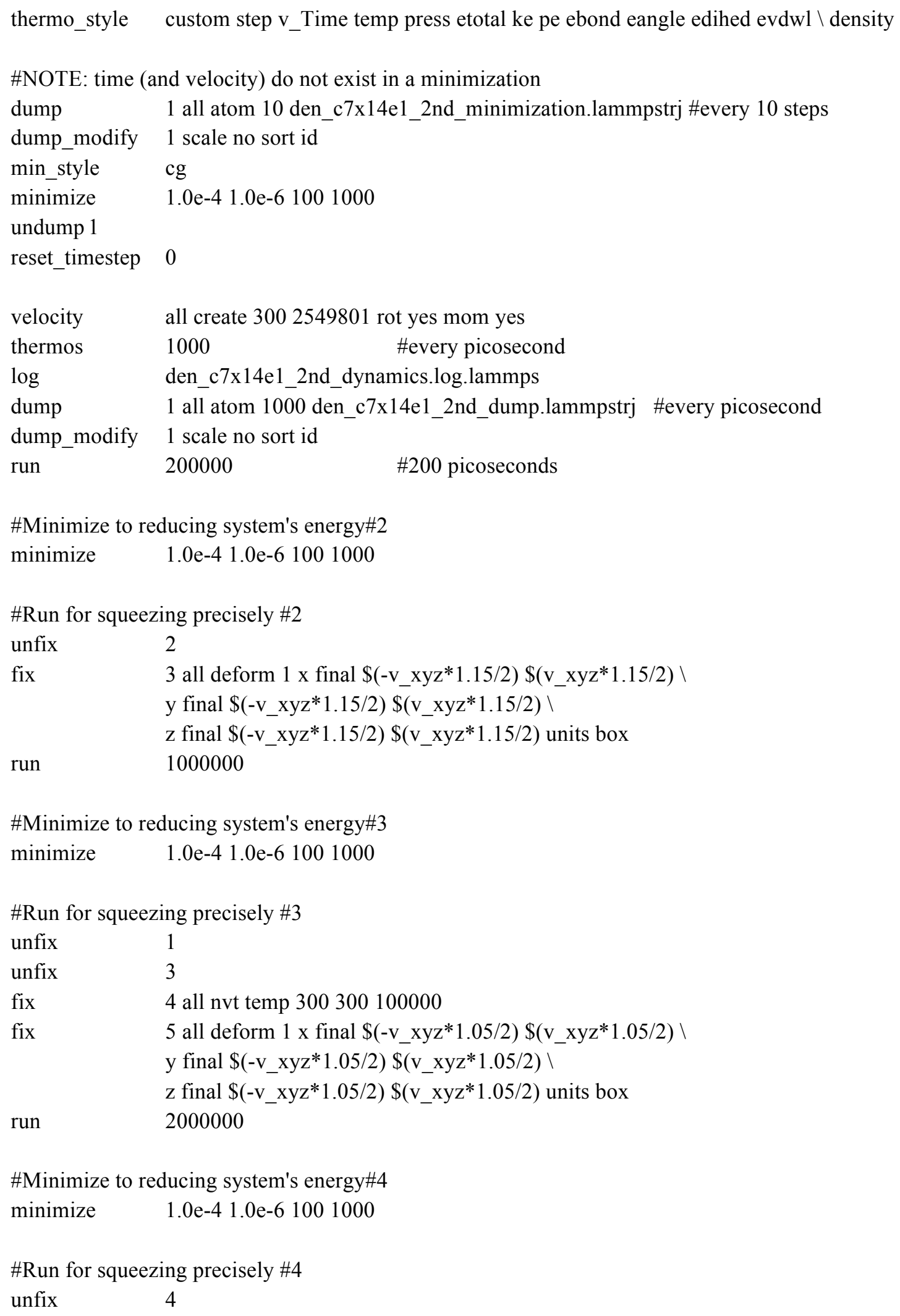




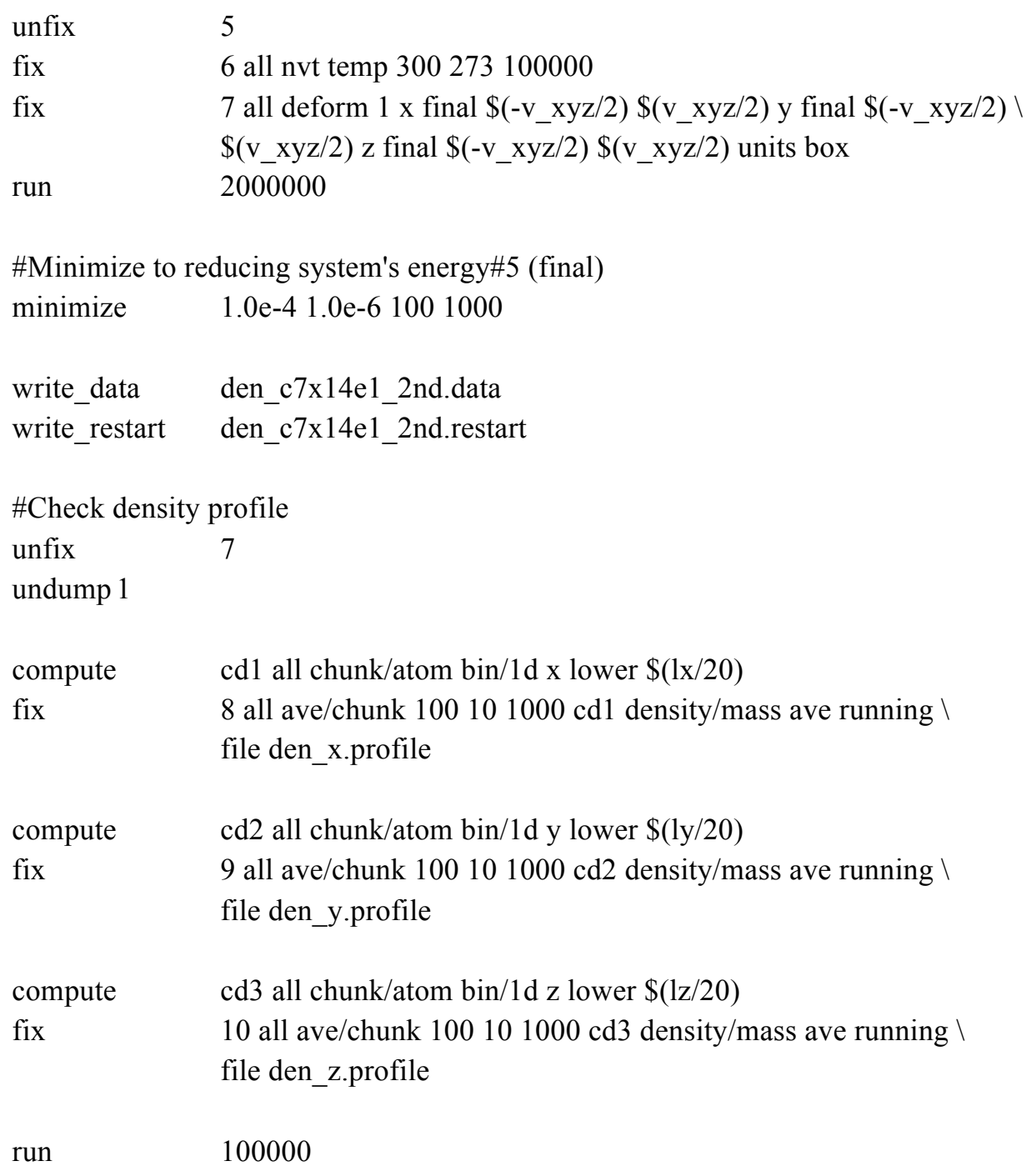




\section{C.2 Crosslink Scripts Package for pure CE}

The crosslink script package includes initial crosslink scripts (LAMMPS), bond-updating scripts (Python), angle and dihedral-updating script (Python), relaxing script(LAMMPS), and control script (Bash).

\section{C.2.1 Initial crosslink script (LAMMPS)}

The crosslink script is for creating the initial bond shown as following.

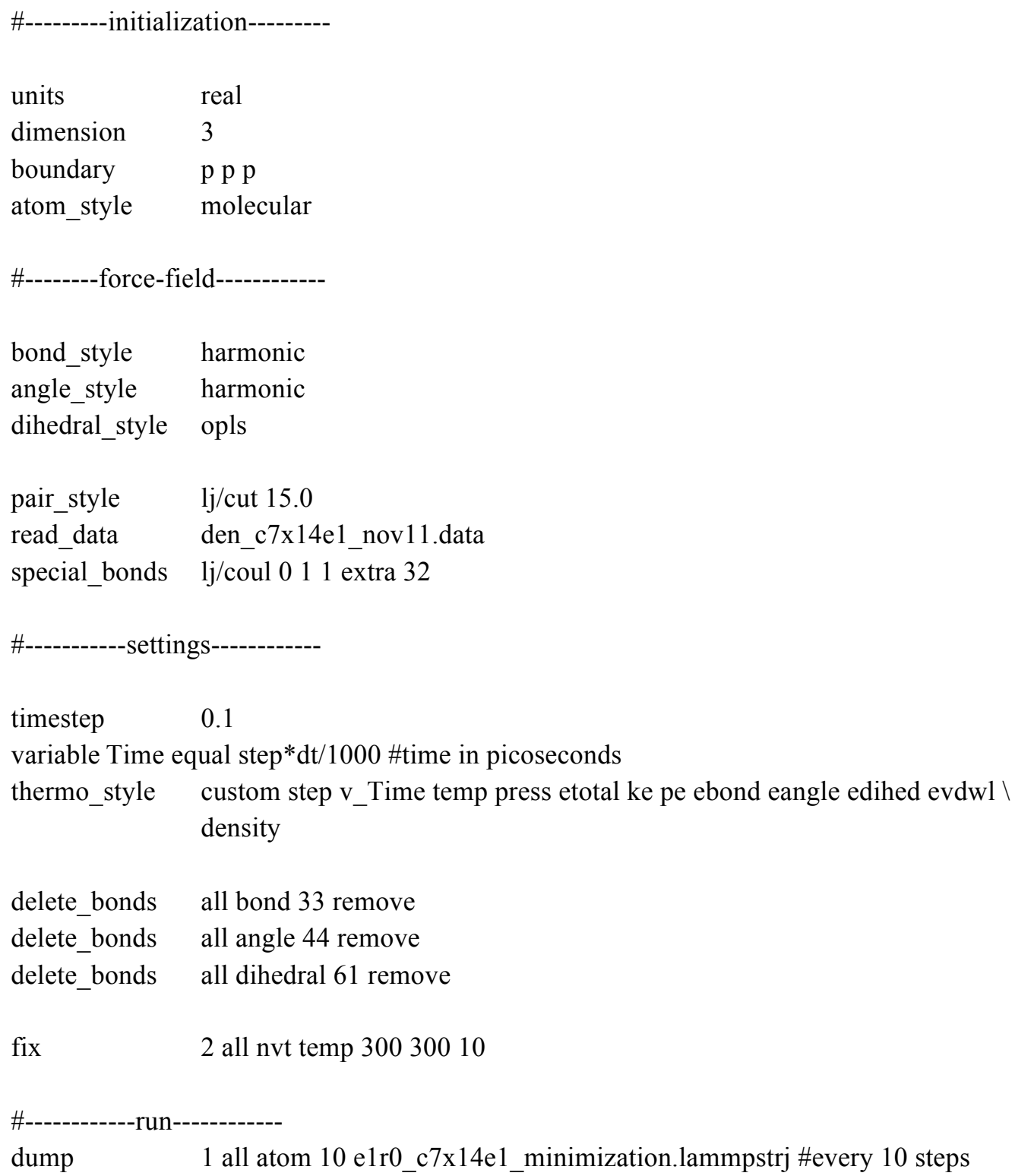




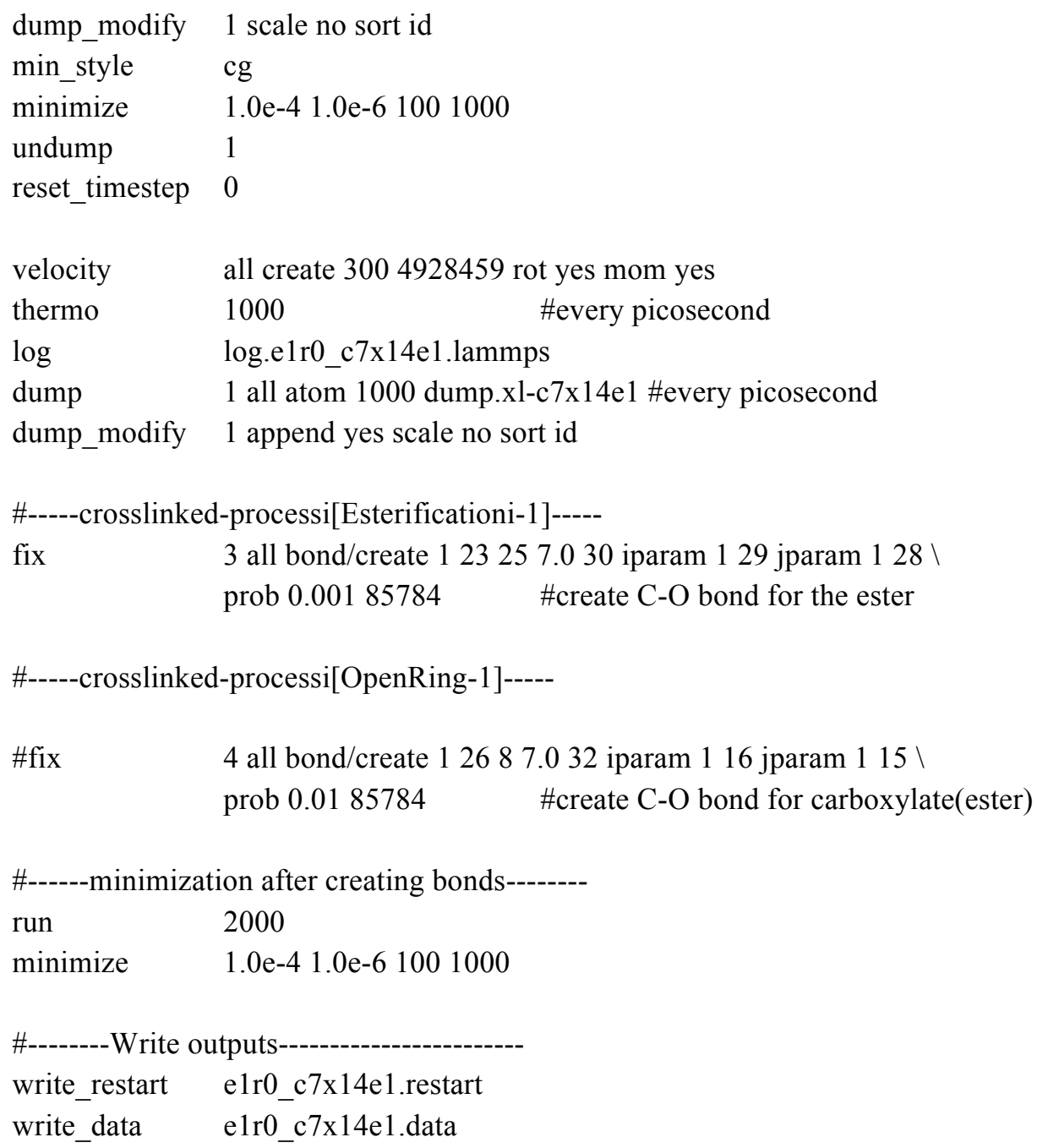

\section{C.2.2 Angle and dihedral-updating script (Python)}

This package can be downloaded from:

https://github.com/schinkan/PhD_research/tree/master/BAD_script 


\section{C.2.3 Relaxing script(LAMMPS)}

The script for relaxing the model during crosslink.

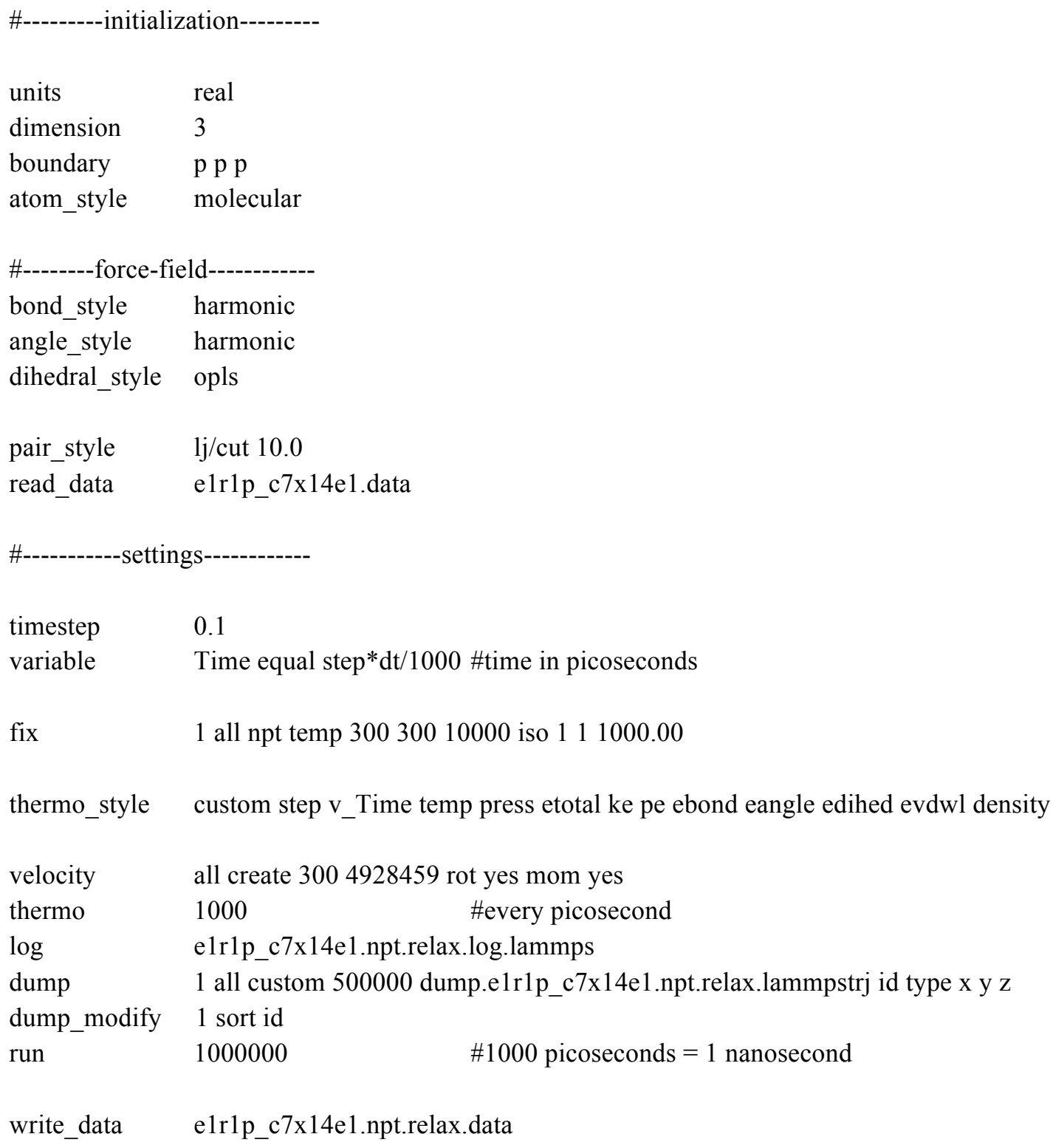




\section{C.3 EMD scripts for pure CE}

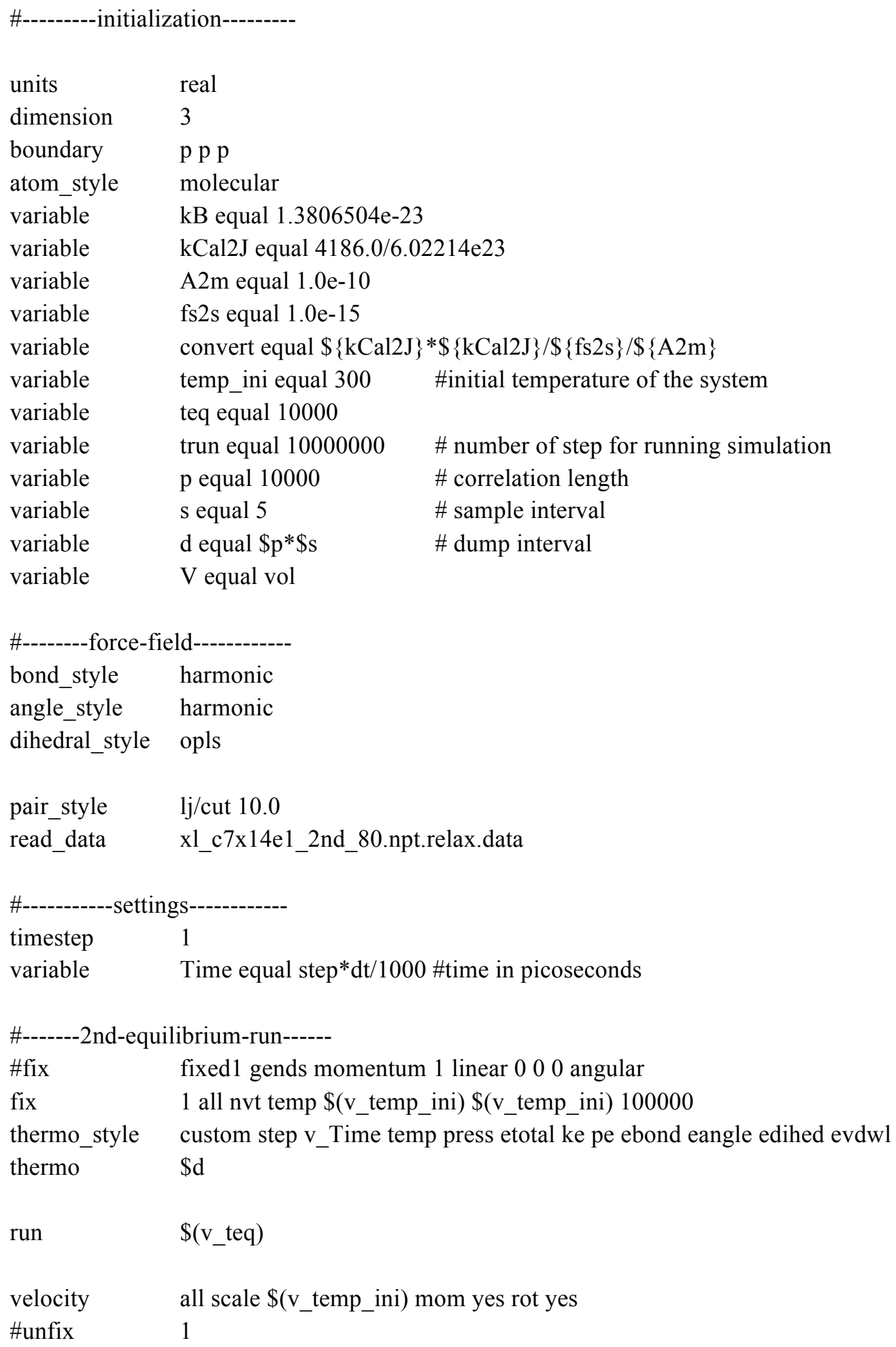




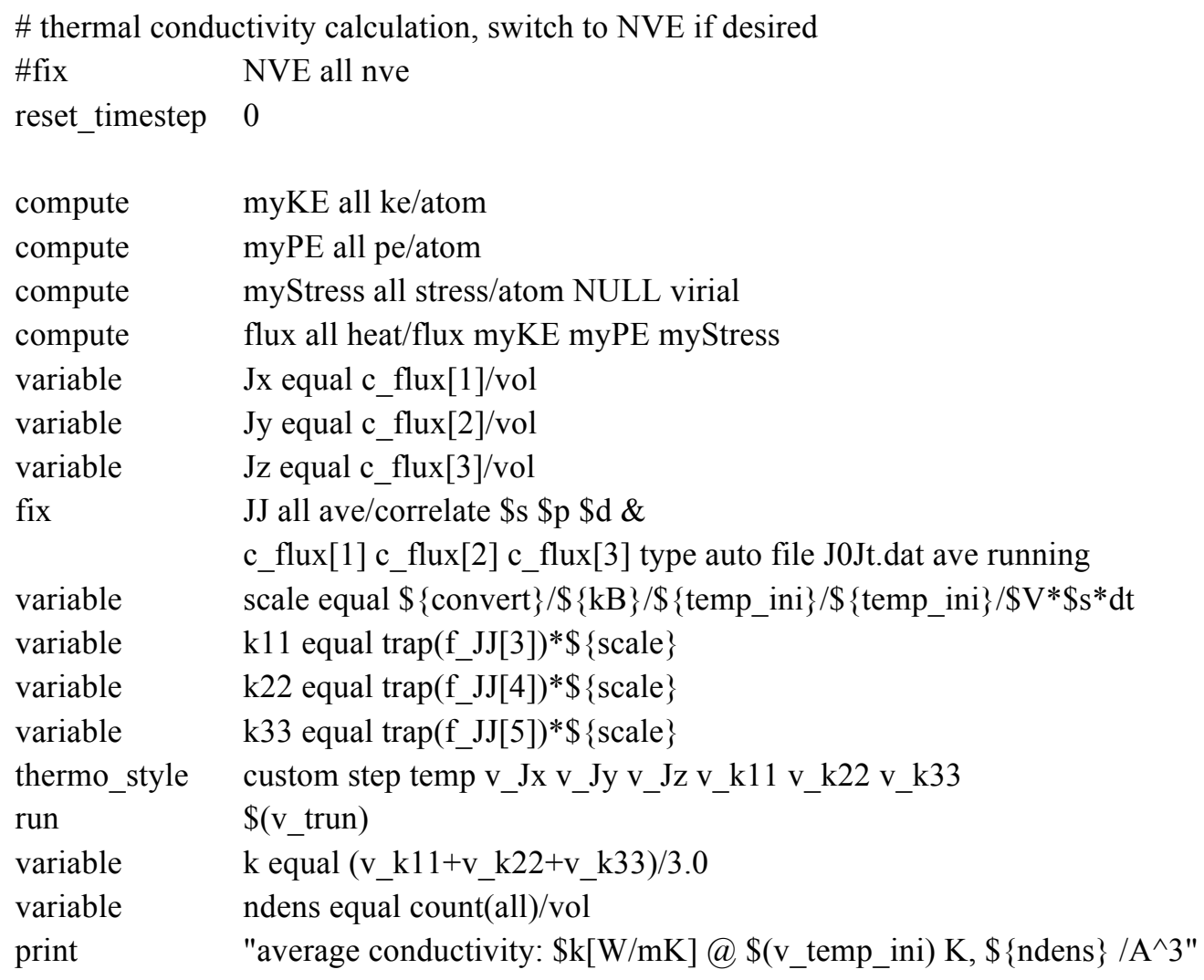

